\title{
The second ROSAT PSPC survey of M 31 and the complete ROSAT PSPC source list ${ }^{\star}$
}

\author{
R. Supper ${ }^{1}$, G. Hasinger ${ }^{1,2}$, W. H. G. Lewin ${ }^{3}$, E. A. Magnier ${ }^{4}$, J. van Paradijs ${ }^{\dagger, 5,6}$, W. Pietsch ${ }^{1}$, \\ A. M. Read ${ }^{1}$, and J. Trümper ${ }^{1}$ \\ 1 Max-Planck-Institut für extraterrestrische Physik, Postfach 1603, 85740 Garching, Germany \\ 2 Astrophysikalisches Institut, An der Sternwarte 16, 14482 Potsdam, Germany \\ 3 CSR and Department of Physics, Massachusetts Institute of Technology, Room 37-627, Cambridge, MA 02139, \\ USA \\ 4 Canada-France-Hawaii Telescope, 65-1238 Mamalahoa Hwy, Kamuela, HI 96743, USA \\ 5 Astronomical Institute "Anton Pannekoek", University of Amsterdam, Kruislaan 403, 1098 SJ Amsterdam, \\ The Netherlands \\ ${ }^{6}$ Physics Department, University of Alabama in Huntsville, Huntsville, AL 35899, USA
}

Received 6 February 2001 / Accepted 2 April 2001

\begin{abstract}
This paper reports the results of the analysis of the second ROSAT PSPC survey of M 31 performed in summer 1992. We compare our results with those of the first survey, already published in Supper et al. (1997). Within the $\sim 10.7 \mathrm{deg}^{2}$ field of view, 396 individual X-ray sources are detected in the second survey data, of which 164 are new detections. When combined with the first survey, this result in a total of 560 X-ray sources in the field of M 31. Their $(0.1 \mathrm{keV}-2.0 \mathrm{keV})$ fluxes range from $7 \times 10^{-15} \mathrm{erg} \mathrm{cm}^{-2} \mathrm{~s}^{-1}$ to $7.6 \times 10^{-12} \mathrm{erg} \mathrm{cm}^{-2} \mathrm{~s}^{-1}$, and of these 560 sources, 55 are tentatively identified with foreground stars, 33 with globular clusters, 16 with supernova remnants, and 10 with radio sources and galaxies (including M 32). A comparison with the results of the Einstein M 31 survey reveals 491 newly detected sources, 11 long term variable sources, and 7 possible transient sources. Comparing the two ROSAT surveys, we come up with 34 long term variable sources and 8 transient candidates. For the M 31 sources, the observed luminosities range from $4 \times 10^{35} \mathrm{erg} \mathrm{s}^{-1}$ to $4 \times 10^{38} \mathrm{erg} \mathrm{s}^{-1}$. The total $(0.1 \mathrm{keV}-2.0 \mathrm{keV})$ luminosity of M 31 is $(3.4 \pm 0.3) \times 10^{39} \mathrm{erg} \mathrm{s}^{-1}$, distributed approximately equally between the bulge and disk. Within the bulge region, the luminosity of a possible diffuse component combined with faint sources below the detection threshold is $(2.0 \pm 0.5) \times 10^{38} \mathrm{erg} \mathrm{s}^{-1}$. An explanation in terms of hot gaseous emission leads to a maximum total gas mass of $(1.0 \pm 0.3) \times 10^{6} \mathrm{M}_{\odot}$.
\end{abstract}

Key words. galaxies: fundamental parameters - galaxies: individual: M 31 - galaxies: spiral - X-rays: galaxies

\section{Introduction}

Before ROSAT, the knowledge of the X-ray properties of M 31 was mainly based on the IPC and HRI observations with the Einstein observatory. These observations were performed in the years $1979 \& 1980$ and the main results are described in van Speybroeck et al. (1979); van Speybroeck \& Bechtold (1981); Long \& van Speybroeck (1983); Crampton et al. (1984); and Trinchieri \& Fabbiano (1991, hereafter TF). In $300 \mathrm{ks}$ of total exposure, $\sim 86 \%$ of the area of our neighbouring spiral galaxy M 31 had been covered to a limiting sensitivity of $\sim 10^{37} \mathrm{erg} \mathrm{s}^{-1}$. Many of the 108 detected point sources

Send offprint requests to: R. Supper, e-mail: ros@mpe.mpg.de $\dagger$ Jan van Paradijs passed away on November 2nd, 1999.

* Tables 5, 6, 7 are also available in electronic form at the CDS via anonymous ftp to cdsarc.u-strasbg.fr (130.79.128.5)

or via

http://cdsweb.u-strasbg.fr/cgi-bin/qcat?J/A+A/373/63 within these Einstein observations were measured with a positional accuracy of about $3^{\prime \prime}$, and were found to be concentrated within a highly confused bulge region and an outer region approximately following the spiral arms. In addition, a variety of possible optical counterparts had been determined, dividing into groups of foreground stars within our own Galaxy, accreting objects and supernova remnants in M 31 and background galaxies seen through the disc of M 31. Additionally, it had been considered that the luminosity distribution of the M 31 disk sources were comparable with that of the bulge sources. The high confusion in the bulge region together with the moderate total number of detected sources made it difficult to justify this statement.

Two deep PSPC surveys of M 31 were performed with the ROSAT X-ray observatory, the first in the summer of 1991, the second in the summer of 1992 (with some follow-up observations in the winter of 1992/1993). Both surveys consisted of a number of observations arranged in raster pointings, covering the whole area of M 31 and 
beyond. The total observation time of $200 \mathrm{ks}$ for each survey, together with the higher sensitivity of the ROSAT telescope pushed the limiting flux to a factor of 10-100 lower than for the Einstein observations. Additionally, several ROSAT HRI M 31 pointings were performed, including a very deep one on the bulge. These were discussed by Primini et al. (1993) and Immler (2000).

The results of the first PSPC survey have been described in Supper et al. (1997, hereafter S97). This work led to the detection of $396 \mathrm{X}$-ray point sources with $(0.1 \mathrm{keV}-2.4 \mathrm{keV})$ fluxes ranging from $\sim 5 \times$ $10^{-15} \mathrm{erg} \mathrm{cm}^{-2} \mathrm{~s}^{-1}$ to $\sim 4 \times 10^{-12} \mathrm{erg} \mathrm{cm}^{-2} \mathrm{~s}^{-1}$. Several tentative identifications with foreground stars, globular clusters, supernova remnants, and galaxies were found, but the majority of the objects remained unidentified. For the sources in M 31, the observed luminosities range from $\sim 3 \times 10^{35} \mathrm{erg} \mathrm{s}^{-1}$ to $\sim 2 \times 10^{38} \mathrm{erg} \mathrm{s}^{-1}$ (at the assumed M 31 distance of $690 \mathrm{kpc}$ used throughout this paper; see Capaccioli et al. 1989). Also this very first survey settled the question of whether a difference between the integrated luminosity distribution of the globular cluster sources in M 31 and the one in our own Milky Way existed, by showing that they were in fact similar. Also, spectral analyses of the 56 brightest sources were presented, confirming their identifications with optical sources. Additionally, a diffuse component within the bulge region was found, its luminosity estimated to be less than $3.2 \times 10^{38} \mathrm{erg} \mathrm{s}^{-1}$.

For 10 of the brightest sources, and for the bulge as a whole, Trinchieri et al. (1999) presented results from spectral analyses based on data obtained with BeppoSAX. They confirmed that most of the sources correlate with globular clusters and found, for all but one, Low Mass X-ray Binary (LMXB) spectra, typical of X-ray sources in globular clusters. Additionally, they also confirmed the presence of two components in the spectrum of the bulge, though they also stated that it is consistent with a superposition of many LMXB spectra (as Irwin \& Bregman 1999 also did using ASCA and ROSAT data). Furthermore they extended the spectral data range up to $\sim 30 \mathrm{keV}$ by making use of the PDS instrument. Because of the lower spatial resolution of BeppoSAX compared with ROSAT, they could not resolve the bulge into individual sources. Garcia et al. (2000) reported the separating of the central source into 5 individual sources using Chandra data. They interpreted one of these sources ( $1^{\prime \prime}$ from the centre) as a possible central black hole, although it shows an unusual (soft) spectrum. In contrast, using ROSAT PSPC observations, Borozdin \& Priedhorsky (2000) found all the resolved X-ray sources in the core of M 31 to be in accordance with LMXB spectra, this after subtracting a soft component (thought to be thermal emission from hot gas) derived from the area around the individual sources.

The second PSPC survey of M 31, described in this paper, is an improvement over the first in terms of its higher spatial homogeneity across the entire disk of M 31. This has resulted here in the detection of $396 \mathrm{X}$-ray point sources, leading to a grand total of 560 individual sources

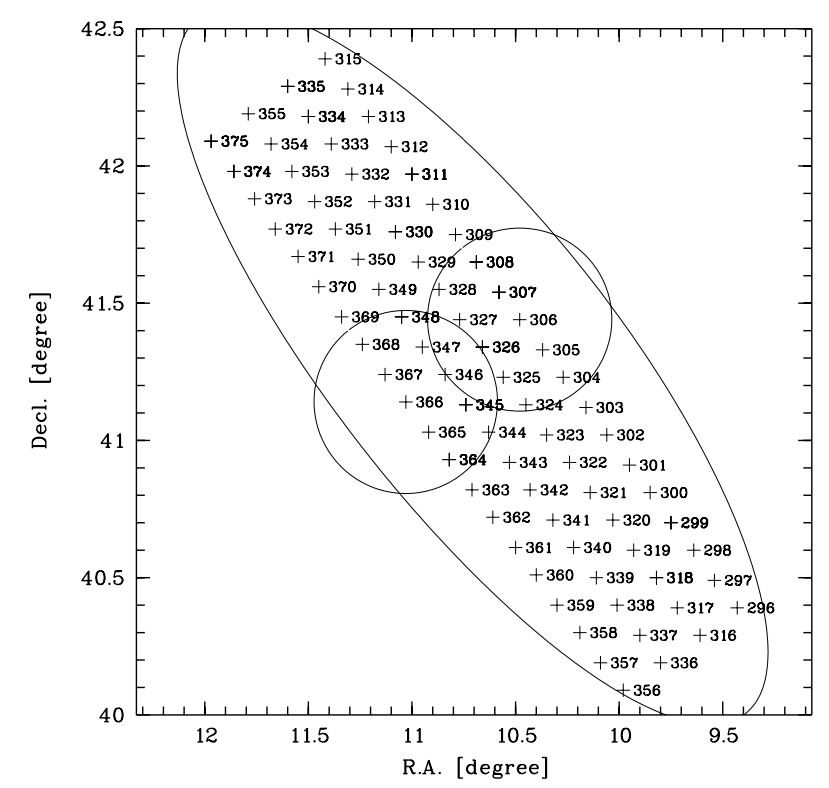

Fig. 1. Locations of the 80 on-axis pointing directions of the second PSPC survey of M 31. The numbers beside the crosses give the last three digits of the corresponding ROSAT observation ID. Two PSPC central regions with $20^{\prime}$ radius are drawn representatively (whereas the total FOV is $\sim 3$ times larger). The $D_{25}$-ellipse of M 31 is indicated.

on merging the two surveys. Both the list resulting from the here-described analysis of the second survey as well as the merged list of both surveys are presented in this paper. For the majority of the X-ray sources already identified in the first survey, the positional accuracy has been improved. Also, the time interval of approximately one year between the two surveys allows a time variability study to be made, and this is also described in this paper. Spectral analysis is not presented here as it is only suitable for the brightest sources, which were already seen in the first survey, their spectra having been studied in S97.

\section{Observations}

The analysis in this paper is based on the second pointed M 31 survey with the ROSAT PSPC, performed in July/August 1992 together with a few follow-up observations in January 1993. This survey consists of $94 \mathrm{ob}-$ servations of 80 different pointing positions, with $2.5 \mathrm{ks}$ total observation time for each pointing. 15 follow-up observations became necessary to complete the observation time of 14 previously interrupted observations and 1 completely failed observation. The details of the observations are given in Table 4 . This entire raster pointing covered the whole disk of M 31 (in terms of its $D_{25}$-ellipse) and more, in 4 strips of 20 pointing directions each. Figure 1 shows the location of these 80 pointings, crosses marking the on-axis directions of the telescope, and the numbers giving the last three digits of the corresponding ROSAT observation ID. Follow-up observations are not numbered as they have the same pointings as their corresponding main observations. For two pointings, a $20^{\prime}$ radius circle 
is drawn to represent the inner area of the PSPC. The instrument's angular resolution and sensitivity are best within this area, though the total field of view (FOV) of the PSPC is $57^{\prime}$ in radius. The $D_{25}$-ellipse of $\mathrm{M} 31$ is also drawn for orientation. Just from this image, it can be seen that the whole of M 31 is covered by the inner PSPC region, leading to an overall approximately constant sensitivity.

The observations were performed in the normal ROSAT wobble mode which adds a slight positional oscillation of $\sim 3^{\prime}$ amplitude on the nominal pointing direction. This was done to smooth out the shadow of the PSPC support structure and to prevent point sources being continually hidden behind the main ribs of the structure. This wobble mode, the finely rastered array of the pointing directions, and the fact that the whole disk of M 31 was covered with the inner PSPC region explain the higher homogeneity of the second survey compared with the first described in S97.

\section{Analysis}

For the analysis, the ROSAT energy range from 0.1 to $2.4 \mathrm{keV}$ was divided into five energy bands: a soft band ( $S: 0.1-0.4 \mathrm{keV})$, two hard bands $(H 1: 0.5-0.9 \mathrm{keV}$ and $H 2: 0.9-2.0 \mathrm{keV}$ ), and two combined bands (hard $H: 0.5$ $2.0 \mathrm{keV}$ and broad $B: 0.1-2.0 \mathrm{keV}$ ). This energy band splitting was used previously in the analysis of the first M 31 survey (S97), except that an upper limit of $2.4 \mathrm{keV}$ was used for the $B$-band. The change from $2.4 \mathrm{keV}$ to $2.0 \mathrm{keV}$ makes no significant difference due to the drastic drop in effective area for the ROSAT telescope + PSPC instrumentation between 2.0 and $2.4 \mathrm{keV}$ (the count rate in the $0.1-2.0 \mathrm{keV}$ energy band is $2 \%$ less than in the $0.1-2.4 \mathrm{keV}$ band, when applying a power law with photon index $\Gamma=-2.0$ and $N_{\mathrm{H}}=9 \times 10^{20} \mathrm{~cm}^{-2}$ as a spectral model - typical for M 31 sources). Therefore the count rates of the two survey analyses are directly comparable.

Parts of the following analysis are based on the Extended Scientific Analysis System (EXSAS; Zimmermann et al. 1993) developed at the Max-PlanckInstitute für extraterrestrische Physik.

\subsection{Data preparation and images}

All the data were inspected for contamination by solar scattered X-rays and particle background. The first originate from Thomson and fluorescent scattering of solar $\mathrm{X}$-ray photons with atoms and molecules in the upper atmosphere along the line of sight. For the ROSAT orbit, these are mainly oxygen, nitrogen, argon, helium, and hydrogen (Jacchia 1972). For the integral solar scatter, the illuminated column density of the atomic oxygen can be used because of the well known fixed ratio of scatter contribution of the other components, as discussed in detail by Snowden \& Freyberg (1993). Therefore, for each pointing, the column density of atomic oxygen was calculated from the orientation of the telescope and the sun position during the whole observation. All time intervals with oxygen column densities above $1 \times 10^{15} \mathrm{~cm}^{-2}$ (see Snowden \& Freyberg 1993 for an explanation of this threshold) were rejected.

Snowden et al. (1992) found a strong correlation between the Master Veto Rate of the ROSAT onboard electronics and the residual particle background not rejected by the veto electronics. Therefore, all time intervals with a Master Veto Rate of more than $170 \mathrm{ct} \mathrm{s}^{-1}$ were additionally rejected. Applying these procedures, the rest of the scattered X-rays and residual particle background within the screened intervals was estimated to be less than $1 \%$.

For the following analysis, the photon events of all 94 observations representing the survey were merged into one single event file. This increased the photon statistics and allowed us to make use of the homogeneity of the raster survey. A slight random offset and rotation of each pointing was corrected for by first correlating bright point sources in neighbouring pointings detected by the Standard Analysis Software System (SASS) and delivered with the data. For this purpose, only sources within the inner PSPC region (20' radius) were used where the telescope has its highest spatial resolution. The final source position was calculated as the weighted mean position from the individual source positions in each contributing pointing, with the signal to noise ratio as the weighting factor. In a last step, each contributing pointing was shifted and rotated to fit best this mean source position. The distribution of the shift and rotation offsets over all 94 pointings was found to be Gaussian-like, with $\sigma=5.2^{\prime \prime}$ in shift and $\sigma=0.21^{\circ}$ in rotation. These corrected data were then ready to be merged.

Figure 2 shows a photon image in the $B$-band from the merged inner PSPC regions of the 94 pointings with a pixel size of $21.5^{\prime \prime} \times 21.5^{\prime \prime}$. Just from this image, the high homogeneity and the narrow (center of detector) point spread function (PSF; Hasinger et al. 1992) of the second ROSAT M 31 survey across the whole galaxy (indicated by the $D_{25}$-ellipse) can be seen, especially when compared to the image of the first survey (Fig. 2 in S97). Some bright identified sources are also indicated in Fig. 2. Most of them are not members of the M 31 system. The bulge region is severely crowded by point sources and confused by an additional diffuse component.

Figure 3 shows an optical image (taken from the Mount Palomar Sky Survey) of M 31 in false colour representation. Size and orientation are as in Fig. 2 and the $D_{25}$-ellipse of M 31 is also marked. The white boxes mark the $560 \mathrm{X}$-ray source positions from the analyses of both ROSAT PSPC surveys of M 31 as described in Sect. 3.3 and listed in Table 6 . The 4 box sizes indicate the logarithm of the X-ray luminosities below 36, between 36 and 37 , between 37 and 38 , and above 38 (from small to large). This corresponds to flux thresholds of $1.76 \times 10^{(-14,-13,-12)} \mathrm{erg} \mathrm{cm}^{-2} \mathrm{~s}^{-1}$. For flux calculations, a spectral model of a power law with photon index $\Gamma=-2.0$ and $N_{\mathrm{H}}=9 \times 10^{20} \mathrm{~cm}^{-2}$ has been used which holds for M 31-sources but not for foreground or 


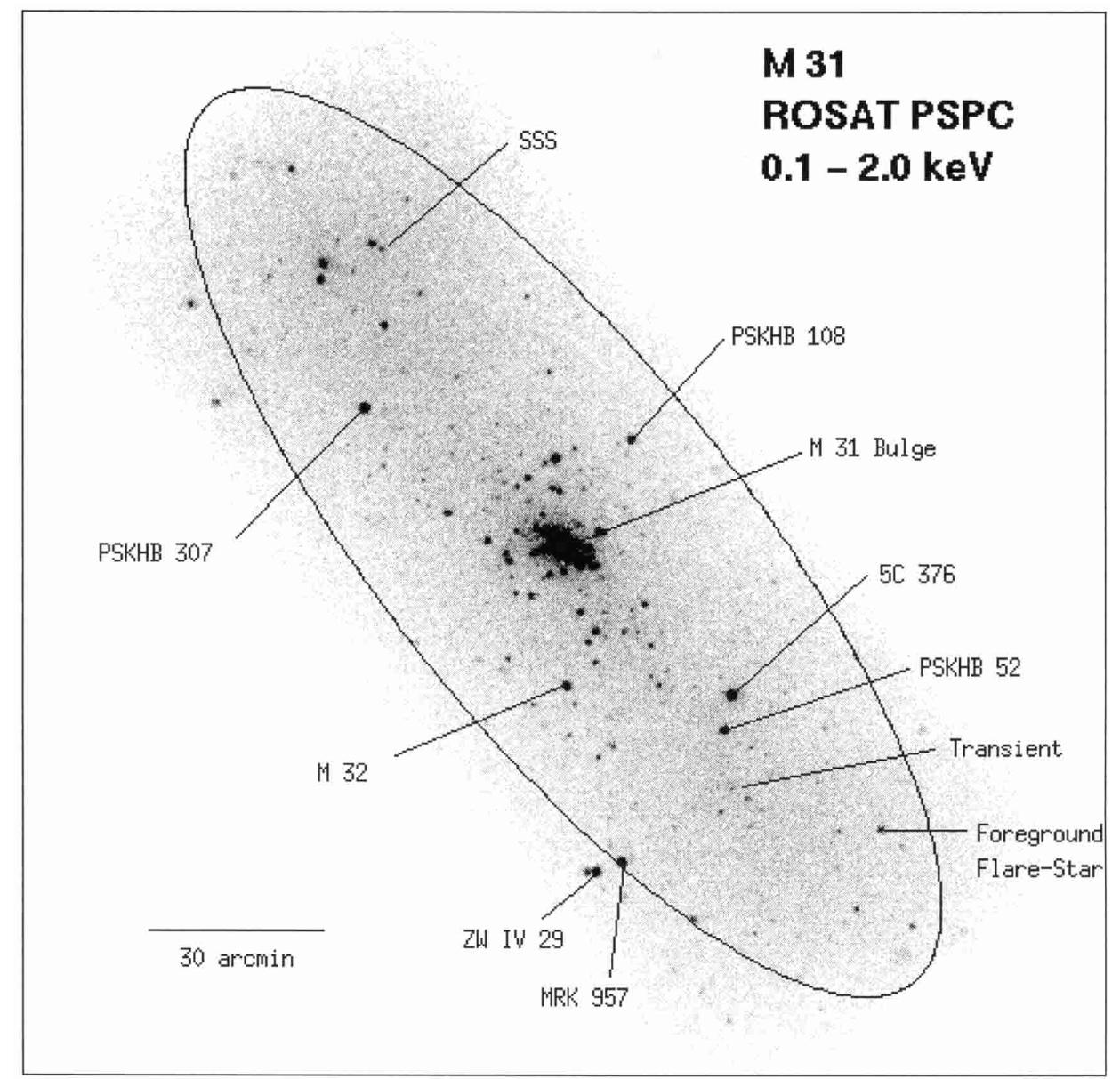

Fig. 2. Projection of the B-band photons of the merged 94 pointings in the inner PSPC regions (20' radius each) with a pixel size of $21.5^{\prime \prime} \times 21.5^{\prime \prime}$. The $D_{25}$-ellipse of M 31 is marked. For a few bright sources their identifications are given, "SSS" standing for "supersoft source". During the first ROSAT M 31 survey, the here indicated transient source was as bright as PSKHB 52.

background objects. A distance of $690 \mathrm{kpc}$ for $\mathrm{M} 31$ is asumed for the resulting luminosity values.

\subsection{Source detection}

To make use of the high homogeneity of the second PSPC M 31 survey, the source detection was performed on the merged data of the inner PSPC regions of all 94 observations. This guaranteed the best results for the determined source positions and covered approximately the whole $D_{25}$-area of $\mathrm{M} 31$. For detections of sources outside this region, the following source detection procedure was repeated using the merged data of the total FOV. The source detection technique used is similar to the one previously used for the analysis of the first survey and described in detail in S97. Hence, only a brief description will be given here, with emphasis on the differences employed.

The computations can be divided into three steps: a local, a map, and a maximum likelihood detection algorithm. For the local detect algorithm, the merged photon event tables were split into a northern, middle and southern part and for each part, images were created with a pixel size of $15^{\prime \prime} \times 15^{\prime \prime}$ for each of the five energy bands. This led to $3 \times 5=15$ images for the three regions and the five energy bands. With a sliding window technique $(3 \times 3$ pixel box $)$, the images were searched for a significant count excess within the box compared with the surroundings. Only source candidates with a likelihood of existence $\geq 8$ were listed, where the likelihood $L=-\ln (P), P$ being the probability that the measured number of photons in the box originate from Poissonian background fluctuations.

In the following map detect algorithm, the same procedure was applied to the 15 images, but this time the photon number within the box was compared with the number of photons within a box of equivalent area and position in a background map. These background maps were computed from the photon images by punching out holes at the source positions determined by the local detect algorithm, and applying smoothing procedures before and afterwards as described in S97. The radius of the holes was set to twice the FWHM of the PSF computed for a $20^{\prime}$ off-axis angle and for the lowest energy value within the considered energy band (a $40^{\prime}$ off-axis angle was used for the merged total FOV data). This resulted in a second list of source candidates (also with $L \geq 8$ ).

For the third step, the local and map source candidate lists were merged into one list (separately for each of the 


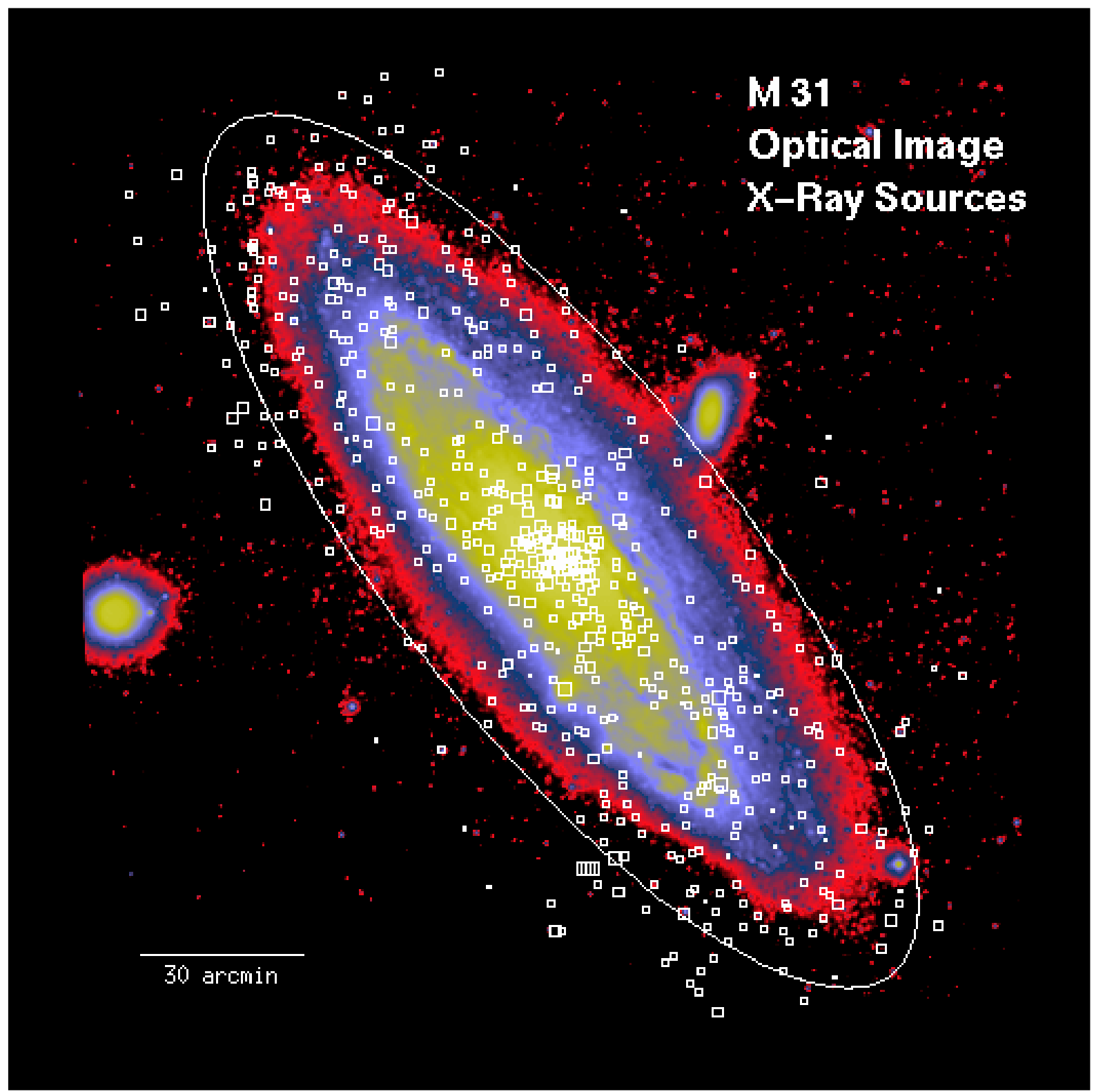

Fig. 3. False colour image of M 31 made from an optical photography with the Mount Palomar observatory. Size and orientation are as in Fig. 2 (also the $D_{25}$-ellipse of $\mathrm{M} 31$ is marked). The white boxes give the 560 X-ray source positions from the analyses of the first and second ROSAT PSPC survey of M 31 as described in Sect. 3.3 and listed in Table 6. The 4 box sizes indicate the logarithm of the X-ray luminosities below 36, between 36 and 37, between 37 and 38, and above 38 (from small to large). This corresponds to flux thresholds of $1.76 \times 10^{(-14,-13,-12)} \mathrm{erg} \mathrm{cm}^{-2} \mathrm{~s}^{-1}$.

five energy bands) and used as input for a maximum likelihood detection procedure (Cruddace et al. 1988). Here only sources with a likelihood $L \geq 10$ were accepted and the background maps described above were used. All resulting lists were merged into one final list such that sources separated by less than $2 \sigma$ of the PSF (referring to the lowest energy value within the considered energy band) were substituted by one single source, its position set to the position of the original source with the highest likelihood. This list was used as input for a repeated maximum likelihood process to compute upper limits in the energy bands where a source was below our detection threshold (but above in any of the other energy bands).

\subsection{The catalogue of detected $X$-ray sources}

The source detection yielded the 396 sources listed in Table 5, which has the same structure as the first survey source list given in Table 5 of S97. Column 1 gives the source number. Columns $2-7$ list the centroid position (epoch J2000) after correction for a systematic offset (see below) and Col. 8 shows the $1 \sigma$ uncertainty of the source position in arcseconds. The calculation of this positional uncertainty is based on the maximum likelihood algorithm and incorporates the effects of statistical errors depending on the number of source counts, together with the blur radius of the PSF at the off-axis angle and the mean photon 
energy of the source. We also set a minimum threshold of $5^{\prime \prime}$ to account for a systematic positional error. The parameter in Col. 9 represents a classification of the quality of the detection and is differently defined than for the first survey due to the different homogeneity and sensitivity of the second survey: class " 1 " indicates sources detected in the inner PSPC region (20' radius) and class "4" sources outside this region. Column 10 in Table 5 gives the highest likelihood of existence found in any of the five energy bands computed with the maximum likelihood method. Finally, Cols. 11 to 15 list the count rates with their $1 \sigma$ errors (in counts per kilosecond) within the five energy bands ( $B, S, H, H 1$, and $H 2$; see beginning of Sect. 3 ). The listed count rate errors are only statistical errors, whereas the systematical errors are expected to be less than $\pm 15 \%$. Because some faint sources were not detected in all energy bands (i.e., these sources had a likelihood below the threshold value of 10 in one or more energy bands), we present $1 \sigma$ upper limits to their count rates. The upper limits are computed from the $1 \sigma$ fluctuations (Poissonian statistics) of the background counts at the source position and are indicated by a preceding "<" symbol.

The 396 X-ray sources found in the second PSPC survey underwent a correlation with a positionally accurate (optical) reference catalogue to determine a systematic offset in source position. This was done in the same manner as for the sources in the first survey, and is described in detail in S97. Here, for reference, we also used the optical globular cluster catalogue of Magnier et al. (1994a; Table 2) which revealed a slight systematic offset in our source positions of $\Delta \mathrm{RA}=5.8^{\prime \prime}$ and $\Delta \mathrm{Dec}=1.2^{\prime \prime}$. Table 5 lists the offset-corrected source positions.

The fact that the total number of detected sources in the second PSPC survey is identical with the total number of detected sources in the first PSPC survey (S97) is purely accidental. The source lists are different and contain only 239 common sources. The detection of common sources in the two surveys is due to the fact that approximately the same region of sky was observed over (in some areas) similar integrated exposure time. The differences in the source lists are mainly due to different sensitivity characteristics: the first survey has its highest sensitivity along a line following the main axis of the M 31 ellipse, whereas the second survey has an approximately constant and high sensitivity across the whole galaxy. Therefore, the detected sources are concentrated within different regions of each survey. Additionally, the slight differences in the source detection procedures and statistical fluctuations cause some departures close to the detection threshold.

Merging of the two survey lists (see Sect. 4.1.1 for details) yielded a final catalogue containing 560 PSPC detected X-ray sources in the field of M 31 . This is presented in Table 6 , which has a similar structure to Table 5 described above. The only differences are that Col. 1 gives the RXJ-number of the source and Col. 2 lists the source number of the first survey (as listed in Table 5 of S97) if a correlation was found. Here four possible cases are indicated; (i) number followed by "+": source was found in
Table 1. Summary of the correlation analysis. $N_{\text {total }}$ gives the number of all possible correlations within a distance of $2 \sigma$ of the combined positional error, $N_{\text {acc. }}$ gives the number of statistically expected accidental correlations, and $N_{\text {fin. }}$ gives the final accepted correlations. For a detailed explanation see Sect. 4.2 .

\begin{tabular}{llrrr}
\hline Type & Databases & $N_{\text {total }}$ & $N_{\text {acc. }}$ & $N_{\text {fin. }}$ \\
\hline X-ray & Einstein $(\mathrm{TF})$ & 82 & 12.7 & 69 \\
GC & BA87, BA93, MA94a & 43 & 11.6 & 33 \\
Extragalactic & NED & 10 & 0.6 & 10 \\
Foreground & MA92, SIMBAD & 72 & 40.4 & 55 \\
SNR & DO80, BW93, MA95 & 22 & 4.1 & 16 \\
Novae & SA91, SA92 & 0 & 0.8 & - \\
\hline
\end{tabular}

both surveys and the listed data are from the first survey, (ii) number followed by "-_": source was found in both surveys and listed data are from the second survey, (iii) number without any additions: source was found only in the first survey, the listed data being from there, and (iv) no number at all: source was found only in the second survey, the listed data being from there. For the criteria of which data are listed in cases of correlation see Sect. 4.1.1. The following Cols. 3-16 are identical with Cols. 2-15 of Table 5 , and have been described above. For sources found in the first survey, the classification parameter listed in Col. 10 is as follows: class "1" identifies sources detected in the central region of the PSPC with off-axis angles $\leq 20^{\prime}$, class "2" defines locations of sources found between $20^{\prime}$ and 40 ', and class " 3 " contains sources with off-axis angles $>40^{\prime}$. As mentioned in Sect. 2 of S97, the source position was derived from the pointing in which it appears at the lowest off-axis angle, i.e., the best class (though not under a PSPC rib). For sources in class " 2 " and especially class "3", any upper limit in count rate listed in Cols. 11-15 can even be an underestimation due to the wider PSF and the therefore higher possibility of rib influencies. For sources found in the second survey, the listed classification parameter for the quality of detection is defined as described above: class " 1 " for sources detected in the inner PSPC region (20' radius) and class " 4 " for sources outside this region.

The caveats for the first survey source catalogue mentioned in S97 are still valid where these sources are not substituted by second survey detections.

\section{Comparisons with other source catalogues}

For all correlations with other catalogues described in this section, the final source list of Table 6 was used. Table 1 summarises the results of the correlation analysis for different catalogues and these are discussed in more detail in the following subsections. From the description (in S97) of the correlation process itself, we simply summarise here that it yields not only the total number of correlating sources $\left(N_{\text {total }}\right)$ but also the amount of expected accidental correlations $\left(N_{\text {acc. }}\right)$ within a $1 \sigma$ confidence level. 


\subsection{Comparisons with previous $X$-ray source catalogues}

\subsubsection{Comparison with the first ROSAT survey of M 31}

The source list of the second M 31 survey was merged with that of the first to obtain the final ROSAT PSPC X-ray source list of M 31 (Table 6). For this purpose the above-mentioned correlation process was applied to both lists to identify common sources. The "radius of acceptance" $\left(r_{\mathrm{a}}\right)$ - the important correlation parameter was iteratively determined as follows: the correlation procedure was repeatly carried out, with $r_{\mathrm{a}}$ increasing successively from $r_{\mathrm{a}}=\sigma_{\text {comb. }}$ (here, $\sigma_{\text {comb. }}=\sqrt{\sigma_{1}^{2}+\sigma_{2}^{2}}$, the combined positional error of the correlating sources, where the single positional error of each source is given by the maximum likelihood detect algorithm). This iterative process was stopped just before the occurrence of multi-identifications (one source in one catalogue correlating with more than one source in the other catalogue) for sources with likelihood $\geq 20$ (to exclude sources near the detection threshold), and outside confused regions. In this way, most potential common sources have been uncovered, without risking having to accept multi-identifications for bright isolated point sources.

This process yielded 239 correlations (with $r_{\mathrm{a}}=$ $4 \sigma_{\text {comb. }}$, corresponding to a $99.99 \%$ probability of all real identifications having been found) with an expected number of 23 chance coincidences ( $1 \sigma$-value). A few multicorrelations were accepted (see below) because they either occur within confused regions or have likelihoods $<20$ or at least one of the correlating sources is covered by the PSPC rib structure.

For sources correlated within the two surveys, the one with the better quality of detection (i.e. with the lowest classification parameter listed in Col. 10 of Table 6, class "4" of the second survey being considered the same as classes "2" and "3" of the first) was taken and the other was rejected as being identical with the first. In cases where the correlating sources were of the same class, the one with the higher likelihood was taken and, if the likelihood was also the same, the source from the second survey was taken because of its better positional accuracy ${ }^{1}$. The same procedure was applied to the few multicorrelations to clarify their situation. With this only one multi-correlation remained: source \#379 of the first survey (see Table 5 in S97) correlates with sources \#380 and \#384 of the second survey (see Table 5 in this paper). Applying the rules mentioned above for both correlations we would have to accept source \#379 from the first survey and would have to reject both sources from the second

\footnotetext{
1 As a concequence of this data quality based decision variable sources are preferentially listed in their luminous state which may have different spectral characteristics compared with their less luminous state. Therefore sources detected as variable (see Tables 2 and 3) are marked with a " $\sim$ "-sign in front of their RXJ-number.
}

survey. Here we decided only to accept the correlation with the least distance as a true identification and left source \#384 as a new one.

Conversely, 158 sources from the first survey and 163 from the second do not correlate with any other source (and we extend to 164 for the second survey due to the reasons mentioned above). To consider all these sources as transients would ignore the different spatial sensitivity distributions and different sky coverage of the two surveys. Therefore, a more explicit investigation of transients is presented in Sect. 5.

\subsubsection{Comparison with the Einstein catalogue}

The $560 \mathrm{X}$-ray sources in the merged source list of the two ROSAT PSPC surveys exceeds the number of X-ray sources detected with the Einstein observatory in this region of sky by a factor of more than 5 . On the one hand, it is the result of the $\sim 10$ times higher sensitivity of ROSAT and the larger exposure of the disk region in the second ROSAT survey. On the other hand, both ROSAT surveys covered a more complete and therefore larger portion of the M 31 field than the Einstein observations did. The number of sources detected with the ROSAT PSPC in the M 31 bulge region (within $1 \mathrm{kpc}$ from the centre) increased from 22 in the first survey to 31 using the data from both surveys. The fact that this is still less than the 48 sources found with the Einstein observatory in this region, as listed by Trinchieri \& Fabbiano (1991, hereafter TF), is due to the large fraction of sources in TF's list which were detected with the higher spatial resolution Einstein HRI. Primini et al. (1993) reported 45 sources found with the ROSAT HRI within the bulge region of M 31 and Immler (2000), again using the ROSAT HRI observations, counted 63 sources within a $5^{\prime}$ circle around the centre.

As already described in S97, the list of Einstein X-ray sources in the field of M 31 reported by TF contains 108 sources, with 81 sources taken from the Einstein HRI data with an assumed positional error of $3^{\prime \prime}$ (reported by Crampton et al. 1984), and 27 sources based on Einstein IPC data with a $45^{\prime \prime}$ positional error. Applying the above mentioned correlation procedure to the 560 ROSAT sources and the 108 Einstein sources reported by TF yields $N_{\text {total }}=82$ correlations with a probable contamination of $N_{\text {acc. }}=12.7$ chance coincidences, here accepting a source separation of up to twice the combined positional error $(2 \sigma) .12$ ROSAT sources each correlated with several Einstein sources, due mainly to the large positional error of the Einstein IPC. To clarify their situation, only the correlation with the smallest separation (between the correlating counterparts) was taken into account. This reduced the number of finally accepted correlations to 69 , which is in good agreement with the number of statistically expected true correlations (i.e. $N_{\text {total }}-N_{\text {acc. }}=69.3$ ).

All 69 identifications are listed in Table 7. Column 1 gives the ROSAT RXJ-number (ref. Table 6), Col. 2 gives 
the fluxes and $1 \sigma$ errors of the ROSAT sources using the spectral model of TF (thermal bremsstrahlung with $k T=5 \mathrm{keV}$ and $N_{\mathrm{H}}=7 \times 10^{20} \mathrm{~cm}^{-2}$ in the $0.2-4.0 \mathrm{keV}$ energy band), Col. 3 lists the Einstein source numbers (ref. Table $2 \mathrm{~A}$ of TF), Col. 4 the fluxes and $1 \sigma$ errors given by TF, and Cols. 5 and 6 the distances between the ROSAT source positions and the Einstein source positions in arcseconds and in units of their combined positional errors $(\sigma)$ respectively. The last column shows the ratio between the fluxes obtained with ROSAT and Einstein and can be considered as a long term variability check between the epochs of the two observations. More detailed investigations into long time variabilities are described in Sect. 5 .

Comparing this correlation list to the one using only ROSAT sources found in the first survey as published in S97 (Table 6), a few remarks should be made. Using only the data of the first survey we had to manually extend the correlation list by one entry (ROSAT source \#67 correlating with Einstein source \#3) as mentioned in S97. This had been necessary because of the poorly-known PSF and the therefore uncertain positioning at the source position. The second PSPC survey now gave us the opportunity to determine much more precisely the position of this source (RX J0040.2+4050), turning out in fact to be only $3.6^{\prime \prime}$ away from the position of the Einstein HRI source \#3. Therefore no manual extension of the correlation list had to be made in this paper.

The listed flux ratio $\left(F_{\mathrm{R}} / F_{\mathrm{E}}\right)$ between ROSAT and Einstein which can be used as a long term variability indicator should be inspected carefully for sources in the bulge region (marked with a $\star$ preceding the ROSAT source number). Because of the heavy confusion in this region, the flux determination of these sources is very uncertain.

With the help of the second PSPC survey, some positions of X-ray sources already found in the first survey could be improved. Therefore, the 69 identifications listed in Table 7 show a very good positional agreement between the PSPC source positions and the ones listed by TF, which were largely obtained with the Einstein HRI. In fact, the mean source separation of the 43 ROSAT PSPCdetected sources correlating with sources also found with the Einstein HRI is $5.9^{\prime \prime} \pm 3.2^{\prime \prime}$.

Excluding the heavily confused bulge region and the sources therein, we found a good ROSAT confirmation $(90 \%)$ of the sources detected with the Einstein observatory as, out of the 60 of the 108 Einstein sources outside the bulge region, 54 could be confirmed by ROSAT. For the 6 Einstein-only detected sources, we give ROSAT flux upper limits and discuss their transient nature in Sect. 5.1. Over and above this, 491 new sources have been found with ROSAT which were not detected with Einstein.

\subsection{Correlations with optical and radio sources}

To identify and classify individual sources, the merged ROSAT source list of both surveys (Table 6) was correlated with the same catalogues previously used for the sources of the first survey in S97. For completeness and to simplify the discussions, we summarise the public data bases and catalogues used as follows:

- globular clusters: the two lists of Battistini et al. (1987, 1993; hereafter BA87, BA93) and the lists of Magnier et al. (1994a; Table 2; hereafter MA94a);

- extragalactic objects: the NASA Extragalactic Database (version date: 30. Dec. 1992; hereafter NED);

- foreground stars: the catalogue of stellar photometry described by Magnier et al. (1992), hereafter MA92, and Haiman et al. (1994) and the SIMBAD catalogue (Centre de Données astronomiques de Strasbourg; version date: Dec. 1989; hereafter SIMBAD);

- supernova remnants: the lists of d'Odorico et al. (1980; hereafter DO80), Braun \& Walterbos (1993; hereafter BW93), and Magnier et al. (1995; hereafter MA95);

- novae: the two lists of Sharov \& Alksnis (1991, 1992; hereafter SA91, SA92).

Information regarding the characteristics of these catalogues, especially the individual positional errors used in the correlation processes, can be found in S97. We adopted them except for the SNR catalogues: D'Odorico et al. (1980) report general position errors of $8^{\prime \prime}$ in declination and $15^{\prime \prime}$ in right ascension. In S97 we assumed a mean position error of $12^{\prime \prime}$ whereas in this paper we decided to use a geometric mean of $17^{\prime \prime}$. For the SNR list of Braun \& Walterbos (1993) and also for the list of Magnier et al. (1995) we used $5^{\prime \prime}$ as a systematic position error for our correlations.

Table 8 shows the result of the correlations. The columns are defined as follows. Column 1 gives the ROSAT RXJ source number (ref. Table 6). Column 2 lists the object class, of which four exist: "Star" for galactic foreground stars followed in brackets by their type if available, "EO" for extragalactic objects, mainly background galaxies, "GC" for sources belonging to globular clusters, and "SNR" for supernova remnants. Column 3 lists the identification, using the abbreviations of the correlated catalogues as defined above. The number following in brackets gives the name/entry number of the object as listed in the relevant catalogue (for details see the remarks to the individual catalogues below). Finally, Cols. 4 and 5 give the distance between the ROSAT source position and the correlated object in the catalogue, both in arcseconds and in $\sigma$ units. For the distance expressed in sigma, the combined positional error of the ROSAT source and the correlated catalogue source was used.

Concerning this list, the following should be noted. If one ROSAT source correlates with more than one catalogue source of the same catalogue, only the correlation with the smallest positional separation is listed. If the correlating catalogue sources belong to different catalogues of the same source class then all correlations are listed, separated by commas. In a few cases, multi-correlations 
between one ROSAT source and catalogue sources of different source classes were found. Here, spectral considerations clarified the situation, especially for distinguishing between foreground stars and globular clusters. Rejections of a good spatial correlation in place of a poorer spatial correlation only took place when the more distant counterpart was spectrally consistent with the ROSAT source and the closer counterpart very inconsistent.

In contrast, no rejection was performed in cases of perfect positional single-correlations, even of moderate coincident spectral characteristics. Additionally, we did not accept identifications with supernova remnants for ROSAT sources with a hardness ratio $H R_{1}+\sigma_{H R_{1}} \leq-0.80$, because we consider these sources as supersoft sources (see S97 and Greiner et al. 1996) $)^{2}$. The hardness ratio $H R_{1}$ is defined as $H R_{1}=(H-S) /(H+S)$, where $S$ and $H$ stand for the source counts in the relevant energy bands calculated with the maximum likelihood algorithm (and listed in Table 6). With these criteria, 114 identifications with optical and radio sources were found, corresponding to an identification quota of $20.4 \%$. Some quantitative comments on the various object classes are as follows:

Foreground Stars (Star): Among the $N_{\text {total }}=72$ correlations within the $2 \sigma$ error level, 17 had to be rejected due to the above criteria, leading to $N_{\text {fin. }}=55$ finally accepted identifications. The high density of foreground stars within the HA94 catalogue yields a relatively high number of possible chance coincidences, $N_{\text {acc. }}=40.3$. The resulting statistically expected number of true identifications is $N_{\mathrm{i}}=31.8 \pm 6.3$, which is too low when compared with the finally accepted 55 identifications. As already discussed in S97, from the Einstein and ROSAT medium and deep surveys we know the foreground source luminosity function, and this can be used to derive an upper limit of 54 expected foreground sources within the region covered by the HA94-catalogue. This value is in good agreement with our finally accepted number of identifications.

Background Galaxies (EO): None of the $N_{\text {total }}=10$ found correlations had to be rejected due to the above criteria. The remaining number of $N_{\text {fin. }}=10$ finally accepted identifications is in good agreement with the statistically expected number of $N_{\mathrm{i}}=9.4 \pm 0.8$. The dwarf galaxy M 32 can be found among the identifications, correlating with ROSAT source RX J0042.6+4052.

Globular Clusters (GC): Within the $2 \sigma$ error level we found $N_{\text {total }}=43$ correlations (with $N_{\text {acc. }}=11.6$ chance coincidences), from which 10 had to be rejected due to the above criteria. The remaining 33 finally accepted identifications are in good agreement with the statistically expected number of $N_{\mathrm{i}}=31.4 \pm 3.4$ true

\footnotetext{
${ }^{2}$ Kahabka (1999) used not only $H R_{1}$ but also $H R_{2}$ and $L_{\mathrm{X}}$

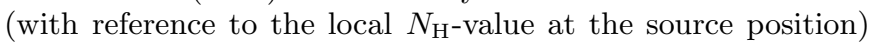
and their respective ratios to discriminate supersoft sources. Using these criteria and the source list of S97, he came up with an additional 26 new supersoft source candidates, 4 of them correlated with foreground stars in S97 and 4 with supernova remnants. Excluding these 8 sources, 18 additional supersoft source candidates in M 31 remain.
}

identifications. Among the 10 rejected correlations, 2 accounted for double-correlations with globular clusters, while the remaining 8 were rejected on spectral grounds, showing soft spectral characteristics incompatible with the known relatively hard spectra of X-ray sources belonging to globular clusters.

Supernova Remnants (SNR): Among the $N_{\text {total }}=$ 22 correlations within the $2 \sigma$ error level, 6 had to be rejected due to the above criteria, leading to $N_{\text {fin. }}=16$ finally accepted identifications. This is in good agreement with the statistically expected number of $N_{\mathrm{i}}=17.9 \pm 2.0$ true identifications. One important comment concerning the SNR-correlations listed in S97: Due to a misuse of the SNR list of Magnier et al. (abbreviated as MA94b in S97) a few SNR miss-correlations are listed in S97. This is repaired in this paper.

Novae: The extension of the ROSAT source catalogue of the first M 31 survey (S97) with the sources found in the second survey does not uncover a single correlation with one of the known novae in M 31.

\section{Time variability}

The two ROSAT PSPC surveys of M 31, separated by $\sim 1$ year, and the Einstein survey from $\sim 11$ years before the first ROSAT survey can be used to search for long term variability within the sources. We treat this here in two different subsections. Readers who wish to investigate long term variabilities or the search for transients should refer to both sections (5.1 and 5.2), and are strongly recommended to read Sect. 4.1 in S97.

Concerning any two catalogues 1 and 2 which refer to the same sources, we used for a quantitative study of possible long term variabilities a linear significance parameter following Primini et al. (1993), which is defined as:

$$
S\left(F_{1}-F_{2}\right)=\frac{\left|F_{1}-F_{2}\right|}{\sqrt{\sigma_{F_{1}}^{2}+\sigma_{F_{2}}^{2}}},
$$

where $F_{1}$ and $F_{2}$ represent the X-ray flux in the first and second source catalogues and $\sigma_{F_{1}}$ and $\sigma_{F_{2}}$ give the corresponding flux errors. This definition is useful in that, in cases where an inappropriate spectral model has been used to compute the two fluxes, any systematic errors are disregarded. We state time variability only for sources with $S \geq 3 \sigma$. Additionally, sources within the bulge and other confused or "handicapped" regions (e.g. beneath the ribs of the PSPC support structure) were excluded on cautionary grounds.

\subsection{Comparison with the Einstein sources}

As already described in Sect. 4.1.2, we compared the complete ROSAT PSPC source list of M 31 (Table 6) with the Einstein source list published by TF. The results are listed in Table 7, where, besides the fluxes (using the spectral model of TF), the flux ratios are also given. Here, 
Table 2. List of X-ray sources showing flux variability between the Einstein observation and the ROSAT observations. $F_{\mathrm{R}}$ gives the ROSAT source flux using the Einstein spectral model of TF (thermal bremsstrahlung with $k T=5 \mathrm{keV}$ and $N_{\mathrm{H}}=7 \times 10^{20} \mathrm{~cm}^{-2}$ in the $0.2-4.0 \mathrm{keV}$ energy band) and $F_{\mathrm{E}}$ gives the Einstein source flux of the correlated Einstein source. Column " $S$ " lists the significance of the variability as described in the text. A "T" in this column indicates bright transients or possible faint transients when enclosed in brackets (see Sect. 5.1 for a detailed explanation).

\begin{tabular}{crrrrr}
\hline $\begin{array}{c}\text { ROSAT } \\
\text { RXJ-No. }\end{array}$ & $\begin{array}{c}F_{\mathrm{R}}\left(\times 10^{13}\right) \\
(\mathrm{cgs})\end{array}$ & $\begin{array}{c}\text { Ein. } \\
\text { No. }\end{array}$ & $\begin{array}{c}F_{\mathrm{E}}\left(\times 10^{13}\right) \\
(\mathrm{cgs})\end{array}$ & $F_{\mathrm{R}} / F_{\mathrm{E}}$ & $S$ \\
\hline $0040.2+4034$ & $19.87 \pm 0.29$ & & $<10.00$ & $>2.00$ & $\mathrm{~T}$ \\
$0041.7+4134$ & $14.38 \pm 0.49$ & 9 & $8.72 \pm 1.08$ & $1.65 \pm 0.21$ & 3.66 \\
$0041.8+4021$ & $24.25 \pm 0.76$ & 11 & $15.54 \pm 0.88$ & $1.56 \pm 0.10$ & 5.69 \\
$0042.2+4019$ & $40.38 \pm 1.23$ & 15 & $48.83 \pm 1.61$ & $0.83 \pm 0.04$ & 3.89 \\
$0042.2+4101$ & $9.53 \pm 0.32$ & 16 & $3.88 \pm 0.75$ & $2.46 \pm 0.48$ & 4.48 \\
$0042.2+4112$ & $9.04 \pm 0.25$ & 19 & $4.26 \pm 0.54$ & $2.12 \pm 0.28$ & 4.45 \\
$0042.2+4118$ & $9.71 \pm 0.32$ & 14 & $3.23 \pm 0.51$ & $3.01 \pm 0.49$ & 6.09 \\
$0042.6+4052$ & $32.45 \pm 0.53$ & 51 & $9.16 \pm 1.01$ & $3.54 \pm 0.40$ & 15.33 \\
$0042.8+4131$ & $18.62 \pm 0.45$ & 67 & $11.95 \pm 1.10$ & $1.56 \pm 0.15$ & 4.30 \\
$0043.1+4118$ & $7.54 \pm 0.24$ & 82 & $2.03 \pm 0.31$ & $3.72 \pm 0.58$ & 6.69 \\
$0043.3+4117$ & $4.44 \pm 0.25$ & 88 & $1.31 \pm 0.34$ & $3.39 \pm 0.89$ & 3.66 \\
$0046.4+4201$ & $10.08 \pm 0.31$ & 105 & $5.52 \pm 0.89$ & $1.83 \pm 0.30$ & 3.33 \\
& SI: $<0.35$ & 12 & $2.56 \pm 0.50$ & $<0.14$ & $(\mathrm{~T})$ \\
& SI: $<0.44$ & 40 & $1.59 \pm 0.62$ & $<0.28$ & $(\mathrm{~T})$ \\
& SII: $<2.01$ & 75 & $4.02 \pm 0.56$ & $<0.50$ & $(\mathrm{~T})$ \\
& SI: $<0.40$ & 84 & $1.99 \pm 0.49$ & $<0.82$ & $(\mathrm{~T})$ \\
& SI: $<0.35$ & 96 & $3.50 \pm 0.94$ & $<0.10$ & $(\mathrm{~T})$ \\
& SI: $<0.39$ & 106 & $0.71 \pm 0.22$ & $<0.55$ & $(\mathrm{~T})$ \\
\hline
\end{tabular}

we extend these calculations by the significance parameter given in formula (1), where catalogue 1 is set to the ROSAT source list and catalogue 2 is set to the Einstein source list. Applying the criteria mentioned above to accept sources only with $S \geq 3 \sigma$ and outside confused regions, we come up with the remaining sources listed in Table 2. Additionally, this table contains potential transients (see below). The meanings of the columns are: Cols. 1 and 3 give the ROSAT source number (RXJ; see Table 6) and the correlating Einstein source (TF's source list) respectively, Cols. 2 and 4 list the (unabsorbed) flux and flux error of the sources as measured with ROSAT and Einstein respectively, the spectral model of TF having been applied (thermal bremsstrahlung with $k T=5 \mathrm{keV}$ and $N_{\mathrm{H}}=7 \times 10^{20} \mathrm{~cm}^{-2}$ in the $0.2-4.0 \mathrm{keV}$ energy band), Col. 5 lists the flux ratio between the ROSAT and the Einstein observations, and Col. 6 gives the significance parameter as described above, or a transient indicator " $\mathrm{T}$ " (see below).

\section{Variable sources:}

Table 2 lists 11 (long term) variable sources. From a comparison between the Einstein detected sources reported by $\mathrm{TF}$ and the sources found in the first ROSAT survey of M 31 we reported 15 potentially variable sources in S97. Actually, 10 of the S97-reported 15 sources vanish from the variability list, and 6 new variable sources join the list. Among the 10 vanished sources, 6 lay within the bulge region (Einstein sources \#33, \#58, \#68, \#76, \#79, and \#80) and have therefore been rejected from our very stringent list (we were not so restrictive for Table 3 of S97). For 2 sources (Einstein sources \#70 and \#348), the fluxes of the corresponding ROSAT sources have been substituted with the data from the second PSPC survey, which were closer to the Einstein fluxes, and the significance of variability therefore fell below our threshold. Einstein source \#2 now correlates with ROSAT source RX J0040.0+4031 (formerly ROSAT source \#55) instead of ROSAT source RX J0040.0+4033 (formerly ROSAT source \#57) because we obtained an improved position from the second PSPC survey data, cancelling the prior correlation. Finally, we deleted by hand the correlation pair of Einstein source \#27 with ROSAT source \#172 because it lies close to the bulge within a confused region.

Among the 6 new variable sources, 3 came into the list due to their newly determined fluxes from the second PSPC survey data (ROSAT sources RX J0041.8+4021, RX J0043.1+4118, and RX J0046.4+4201), 2 joined the list because of the now improved positions of the correlating ROSAT sources (RX J0042.2+4112 and RX J0042.2+4118) and the last one (RX J0043.3+4117) was newly discovered within the second survey data.

In cases where a change in determined flux (between the first and second PSPC survey) is responsible for changes in the variable source list, one should bear in mind that this might be due to a real flux change (variability) of the particular source within the time gap between the two ROSAT surveys ( $\sim 1$ year). In assembling Table 2 , we assumed that the changes are due to the better flux determination within the data of the second PSPC survey compared to the first. Readers who wish to investigate the variable sources are therefore recommended to examine all sources in both lists.

The two variable sources reported by Collura et al. (1990) have been discussed already in S97. Including the second PSPC survey data has added nothing of significance as regards these.

\section{Transients:}

Table 2 lists 7 possible (bright) transient sources. We define bright transients as those sources which are detected in one catalogue, and are bright enough to be detected in the other, but which are not seen. ROSAT sources with fluxes $\geq 10^{-12} \mathrm{erg} \mathrm{cm}^{-2} \mathrm{~s}^{-1}$ (applying the spectral model of TF) should have been seen during the Einstein observations. Conversely, Einstein sources with fluxes $\geq 10^{-12}$ erg $\mathrm{cm}^{-2} \mathrm{~s}^{-1}$ should have been seen in the ROSAT surveys.

From a comparison between the Einstein detected sources reported by TF and the sources found in the first ROSAT survey of M 31, we reported 9 potentially transient sources in S97. In detail, we have now "lost" 5 of these transients, 3 of them because the relevant Einstein sources (\#81, \#93, and \#100) were found to correlate with sources detected within the second PSPC survey data, and the other two because they lay within confused regions. On the other hand, we included 3 new transients in our list (Einstein sources \#12, \#75, and \#84) because, within the first PSPC survey their positions were near 
the PSPC support structure and therefore we formally excluded them from the list at that time. With the help of the second PSPC survey and its more homogeneous exposure, we were able to verify their potential transient nature. For all transients, we list in Table 2 a flux upper limit. In the case of the ROSAT fluxes, we compute these limits from the known background fluxes at the source positions making use of the most sensitive survey (indicated by SI/SII for the first/second PSPC survey). Although 3 sources were partially obstructed by the PSPC support structure within the first survey (see above), for 2 of them we calculated their upper limits from these data because these positions still received more exposure within the first survey than within the second. In these cases, we simply used the second survey and its homogeneity as a proof to clarify their transient nature.

We list all 6 transients at the bottom of Table 2 as faint transients ("T" within brackets) as they have luminosities below our bright transient threshold given above, even though their Einstein luminosities are above the detection threshold of the ROSAT surveys.

For the one transient in Table 2 not seen by Einstein (ROSAT source RX J0040.2+4034), we give our transient threshold of $10^{-12} \mathrm{erg} \mathrm{cm}^{-2} \mathrm{~s}^{-1}$ as an upper limit because $\mathrm{TF}$ did not mention the limiting flux of the individual Einstein observations. With this value, we are surely above the sensitivity of the Einstein observations.

\subsection{Comparison between the two ROSAT PSPC surveys}

In Sect. 3.3 we described the merge of the two source lists assembled from the first and second PSPC surveys of M 31. Sources which were found in both lists have been tested for variability in flux. To indicate a possible variability we have applied the following criteria: (1) The source must reside outside the bulge and outside other confused regions, (2) the significance parameter (Eq. (1)) must hold with $S \geq 3\left(F_{1}\right.$ and $F_{2}$ being the fluxes of the source determined from the first and second surveys), (3) sources with an upper limit to the count rate in the $B$-band in either of the two surveys have been excluded (in other words, the count rate must have been determinable), (4) sources behind/near the PSPC support structure within the first survey have been excluded (i.e. sources marked with a $\dagger$-symbol in Table 5 of S97, (5) the sources have to belong to source class " 1 " in both surveys, and (6) the detection likelihood of the source has to be $\geq 20$ in both surveys. Criterion (1) prevents any pseudovariability occurring due to uncertain flux determinations within confused regions, criterion (2) ensures a sufficient significance, and with criteria (3) to (6), the influence of any systematic errors should be widely excluded.

With these criteria, 34 possible long term variable sources were found, as listed in Table 3. Column (1) gives the ROSAT RXJ-number of the source, Cols. (2) and (3) list the count rate in the $B$-band determined from the
Table 3. List of all the potentially long term variable sources found via a comparison of the first and second ROSAT PSPC surveys of M 31. Column " $S$ " lists the significance of the variability as described in text. Additionally, possible transients are tabled, marked with " $\mathrm{T}$ " in this column.

\begin{tabular}{|c|c|c|c|}
\hline $\begin{array}{c}\text { ROSAT } \\
\text { No. }\end{array}$ & $\begin{array}{c}\text { Rate }_{\text {SI }} \\
\left(\text { ct } * \mathrm{ks}^{-1}\right)\end{array}$ & $\begin{array}{c}\text { Rate }_{\text {SII }} \\
\left(\text { ct } * \mathrm{ks}^{-1}\right)\end{array}$ & $S$ \\
\hline$\overline{\mathrm{RX} J 0038.4+4012}$ & $12.61 \pm 0.66$ & $9.03 \pm 0.781 .40 \pm 0.14$ & 3.51 \\
\hline RX J0040.7+3959 & $<2.81$ & $5.54 \pm 1.01$ & $\mathrm{~T}$ \\
\hline RX J0041.1+4002 & $1.96 \pm 0.64$ & $5.57 \pm 1.010 .35 \pm 0.13$ & 3.04 \\
\hline RX J0041.5+4105 & $<1.27$ & $12.36 \pm 0.69$ & $\mathrm{~T}$ \\
\hline RX J0041.6+4101 & $1.49 \pm 0.31$ & $3.10 \pm 0.40$ & 3.20 \\
\hline RX J0041.8+4015 & $3.18 \pm 0.58$ & $7.02 \pm 1.040 .45 \pm 0.11$ & 3.24 \\
\hline RX J0041.8+4021 & $60.52 \pm 1.22$ & $90.56 \pm 2.850 .67 \pm 0.02$ & 9.68 \\
\hline RX J0041.8+4101 & $11.73 \pm 0.71$ & $6.25 \pm 0.511 .88 \pm 0.19$ & 6.28 \\
\hline RX J0041.8+4122 & $6.11 \pm 0.58$ & $2.69 \pm 0.432 .27 \pm 0.42$ & 4.75 \\
\hline RX J0042.1+4110 & $4.20 \pm 0.46$ & $6.83 \pm 0.650 .62 \pm 0.09$ & 3.28 \\
\hline RX J0042.1+4118 & $13.85 \pm 0.79$ & $35.01 \pm 1.210 .40 \pm 0.03$ & 14.68 \\
\hline RX J0042.2+4039 & $3.90 \pm 0.39$ & $8.12 \pm 0.710 .48 \pm 0.06$ & 5.24 \\
\hline RX J0042.2+4055 & $10.13 \pm 0.69$ & $6.59 \pm 0.521 .54 \pm 0.16$ & 4.10 \\
\hline RX J0042.2+4101 & $35.60 \pm 1.18$ & $29.59 \pm 1.021 .20 \pm 0.06$ & 3.85 \\
\hline RX J0042.2+4112 & $24.95 \pm 1.02$ & $33.74 \pm 0.950 .74 \pm 0.04$ & 6.33 \\
\hline RX J0042.2+4118 & $11.31 \pm 0.73$ & $36.25 \pm 1.210 .31 \pm 0.02$ & 17.64 \\
\hline RX J0042.3+4113 & $18.74 \pm 0.87$ & $66.18 \pm($ & 6.58 \\
\hline RX J0042.4+4104 & $6.95 \pm 0.56$ & $17.85 \pm$ & 0.87 \\
\hline RX J0042.4+4112 & $22.09 \pm 0.94$ & $44.15 \pm 0.760 .50 \pm$ & 18.34 \\
\hline RX J0042.5+4048 & $1.69 \pm 0.32$ & $3.91 \pm$ & 3.97 \\
\hline RX J0042.6+4052 & $121.17 \pm 1.99$ & $58.12 \pm 1.502 .08 \pm 0.06$ & 25.28 \\
\hline $42.8+4125$ & $14.75 \pm 0.84$ & $980.69 \pm 0.05$ & 5.05 \\
\hline RX J0042.9+4146 & $3.55 \pm 0.53$ & $7.64 \pm 0.720 .46 \pm 0.08$ & 4.57 \\
\hline RX J0043.1+4048 & $<2.27$ & $5.53 \pm 0.62$ & $\mathrm{~T}$ \\
\hline RX J0043.1+4112 & $3.06 \pm 0.41$ & $5.63 \pm 0.560 .54 \pm 0.09$ & 3.69 \\
\hline RX J0043.1+4118 & $5.58 \pm 0.57$ & $28.17 \pm 0.910 .20 \pm 0.02$ & 21.05 \\
\hline RX J0043.3+4120 & $6.74 \pm 0.62$ & $0.67 \pm 0.09$ & 3.02 \\
\hline RX J0043.4+4118 & $6.86 \pm 0.62$ & $11.96 \pm 0.760 .57 \pm 0.06$ & 5.20 \\
\hline RX J0043.4+4126 & $<1.50$ & $5.87 \pm 0.52$ & $\mathrm{~T}$ \\
\hline RX J0043.7+4124 & $<1.53$ & $3.72 \pm 0.50$ & $\mathrm{~T}$ \\
\hline RX J0043.7+4136 & $7.04 \pm 0.56$ & $2.16 \pm 0.363 .26 \pm 0.60$ & 7.35 \\
\hline $3.9+4122$ & $4.82 \pm 0.48$ & $2.07 \pm 0.362 .33 \pm 0.47$ & 4.56 \\
\hline RX J0044.3+4145 & $1.17 \pm 0.35$ & $3.07 \pm 0.420 .38 \pm 0.12$ & 3.51 \\
\hline RX J0044.4+4121 & $29.77 \pm 1.12$ & $25.10 \pm 1.021 .19 \pm 0.07$ & 3.08 \\
\hline RX J0044.8+4225 & $<2.74$ & $4.85 \pm 1.11$ & $\mathrm{~T}$ \\
\hline RX J0045.6+4208 & $19.18 \pm 0.92$ & $25.83 \pm 1.110 .74 \pm 0.05$ & 4.63 \\
\hline RX J0045.7+4139 & $147.96 \pm 1.91$ & $134.43 \pm 1.961 .10 \pm 0.02$ & 4.95 \\
\hline RX J0046.4+4201 & $29.58 \pm 1.14$ & $37.64 \pm 1.150 .79 \pm 0.04$ & 4.99 \\
\hline RX J0046.4+4204 & $20.61 \pm 0.99$ & $33.17 \pm 1.160 .62 \pm 0.04$ & 8.24 \\
\hline RX J0047.4+4152 & $<2.08$ & $3.35 \pm 0.54$ & \\
\hline RX J0047.8+4142 & $<2.70$ & $7.02 \pm 1.15$ & $\mathrm{~T}$ \\
\hline RX J0048.4+4157 & $46.28 \pm 1.64$ & $35.30 \pm 2.161 .31 \pm 0.09$ & 4.05 \\
\hline
\end{tabular}

data of the first and second surveys respectively, Col. (4) gives the ratio in count rate between the first and second survey, and Col. (5) gives the value of the significance parameter, following Eq. (1).

Additionally, Table 3 contains possible transients, marked with a "T" in Col. (5). For this, the sensitive flux limit was determined within the survey in which the source was not found, using the source position from the other survey (i.e. where the source was detected). If this value was below the count rate minus the $1 \sigma$ count rate error 
determined from the survey where the source was found, then this source was considered as a possible transient. To prevent false diagnoses being made, the same criteria as above for the variable source search were applied except for criterion (2) which was dropped, and criterion (3), which was substituted as just described. With this, no transients were found which could be seen only in the first survey but not in the second. This is mainly due to the exclusion of regions near the PSPC support structure within the first survey which results in a reduction in area and may have removed a few transient candidates from our (conservative) list. Additionally, the second survey with its homogeneous exposure is more sensitive in the outer region of $\mathrm{M} 31$ than the first survey. As a consequence of these two effects, we found 8 transients which were seen in the second survey but not in the first. Because of the very different conditions of both surveys (mainly the influence of the PSPC support structure in the first survey), we desist from a quantitative analysis of a transient rate and its comparison with expected theoretical values.

If we readopt criterion (2) in a slightly changed form, that the upper flux limit for transient sources also represents the error in flux, we would come up with values for the significance parameter always below our threshold of 3 except for source RX J0041.5+4105 where $S=7.67$. Here, we could quote source RX J0041.5+4105 as a strong candidate for a transient, whereas all the others must be considered as weak candidates.

Some words concerning ROSAT source RX J0040.2+4034: In Sect. 5.1, from a comparison with the Einstein source list of $\mathrm{TF}$, we have indicated this source as a possible transient. If the increase in flux between the Einstein observations and the first ROSAT survey is based on a short-time outburst of this transient source, we would expect this source to appear much fainter during the second PSPC survey or even disappear. Actually, with the criteria applied to merge both source lists as described in Sect. 3.3, the source seemed to disappear, as no correlating source could be found within the second survey. Nevertheless, a visual inspection suggested an identification of ROSAT source RX J0040.2+4034, only found within the first survey (source \#69, Table 5 in S97), with ROSAT source RX J0040.2+4033, only detected within the second survey. Both sources are listed separately in Table 6 . Under the assumption that these two sources are the same source we note a large decrease in count rate (by over a factor of 40) between the first and second surveys. This would tie in with the possible transient nature of this source. On the other hand, the fact that these two sources are separated by $57^{\prime \prime}$ and are both good quality detections argues against this treatement. We therefore list both sources as individual sources in our list.

\section{Total luminosity and diffuse emission}

From the first ROSAT PSPC survey of M 31 we had already derived quantities for the total luminosity of $\mathrm{M} 31$ and a possible gaseous component (S97). Because the first survey had to be corrected for several caveats such as the dominant influence of the PSPC support structure, the inhomogeneous exposure, and the rapid decrease of sensitivity from the centre of M 31 to the outer regions, we improved the determination of the total luminosity and diffuse component with the data from the much more homogeneous second survey. One of the big advantages of the second survey is its more or less constant exposure and therefore constant flux limit over the whole $D_{25}$-area of the galaxy. This allows an improved determination of the background around M 31 and, as a consequence, a more reliable flux determination of components within M 31 . Furthermore, it reduces systematical errors in the case of large scale analysis, as discussed in this section. The following description has some overlap with procedures already described in S97, but we decided to briefly summarise them here for completeness.

In this section we will use the term "diffuse component" to mean the sum of the emission from a truly diffuse (gaseous) emitter and from unresolved point sources. We will refer to "total emission" as the sum of the diffuse component and the emission from resolved point sources.

As already described in Sect. 3.1, all the data have been cleaned of contamination by solar scattered X-rays and particle background. The resulting photon event files remain contaminated by these components, but only to less than $1 \%$ in each pointing. This is up to ten times better than in the worst case of the first PSPC survey. For the analysis in this section, the data were binned into an image with a $30^{\prime \prime} \times 30^{\prime \prime}$ pixel size. For the determination of count rates within the $D_{25}$-area of M 31 , the merged inner regions of the PSPC with $20^{\prime}$ radius have been used, whereas for the outer area around M 31, a merge of the total photon event files has been used. The resulting images were divided by exposure maps with the same pixel size to obtain count rate images corrected for the effects of the rib structure, vignetting and dead time. These exposure maps were calculated in the following manner: the $B$-band was divided into 10 energy slices for which EXSAS provides instrument maps for the PSPC detector response. Together with the photon event files, exposure maps for each of these energy slices were created, considering also dead time effects. A weighted addition of these single exposure maps yields the final exposure maps. The pulse height spectra in the 10 energy slices of the photon event files were used as the weighting factors.

From the image of the merged inner PSPC regions we derived count rates for the bulge $(1 \mathrm{kpc}$ around the centre) and the M 31 disk region (i.e. outside the bulge up to the $D_{25}$-ellipse). "Background count rates" were taken from the image of the merged total PSPC FOV and within an area far outside and around the $D_{25}$ ellipse of M 31 explicitly the area between the ellipse with major and minor axes $0.15^{\circ}$ larger than the $D_{25}$ ellipse of M 31 and the ellipse $0.30^{\circ}$ larger. Sources within this area were cut out to a radius of three times the PSF at the source position. With this, we derived count rates for the bulge, 
disk, and "background" of $(46.86 \pm 2.5),(4.278 \pm 0.04)$, and $(3.311 \pm 0.038) \mathrm{ct} \mathrm{s}^{-1} \mathrm{deg}^{-2}$ respectively, in the broad $(0.1-2.0 \mathrm{keV})$ energy band.

Considering the bulge, a subtraction of the background count rate and a multiplication with the bulge area of $0.026 \mathrm{deg}^{2}$ yields $(1.132 \pm 0.065) \mathrm{ct} \mathrm{s}^{-1}$. Applying a power law with $\Gamma=-2.0$ for the spectral model and a galactic foreground absorption of $N_{\mathrm{H}}=6 \times 10^{20} \mathrm{~cm}^{-2}$ yields $(2.88 \pm 0.17) \times 10^{-11} \mathrm{erg} \mathrm{cm}^{-2} \mathrm{~s}^{-1}$ for the total flux of the bulge region, which corresponds to a luminosity of $\sim 1.6 \times 10^{39} \mathrm{erg} \mathrm{s}^{-1}$, assuming a distance of $690 \mathrm{kpc}$ to M 31. A summation over the count rates of all 22 bulge sources detected in the second PSPC survey data in this area initially yields $(2.78 \pm 0.02) \mathrm{ct} \mathrm{s}^{-1}$. This is much higher than the total emission derived above. The reason is the way the source detection algorithm works. In highly confused regions it tends to overestimate the count rate of each source due to overlapping of the photon extraction circles of neighbouring sources. By determining the individual extraction radii the detection algorithm has used, and the amount of overlapping area under the assumption of a gaussian PSF for the instrumentation, we can globally correct for this effect. With this, we obtain $(0.893 \pm 0.006) \mathrm{ct} \mathrm{s}^{-1}$ for the resolved emission of the bulge. A comparison with the above derived total emission uncovers an unresolved component of $(0.239 \pm 0.065) \mathrm{ct} \mathrm{s}^{-1}$. Assuming that this component completely originates from thermal emission of hot gas, and applying a spectral model for an optically-thin thermal plasma (MEKAL) with $k T=0.35 \mathrm{keV}$ (as determined from XMM-Newton observations, e.g. see Shirey et al. 2001) and a galactic foreground absorption of $N_{\mathrm{H}}=$ $6 \times 10^{20} \mathrm{~cm}^{-2}$, we derive $(3.4 \pm 0.9) \times 10^{-12} \mathrm{erg} \mathrm{cm}^{-2} \mathrm{~s}^{-1}$ for a diffuse X-ray flux. For a distance of $690 \mathrm{kpc}$ to M 31, this corresponds to a luminosity of $(2.0 \pm 0.5) \times 10^{38} \mathrm{erg} \mathrm{s}^{-1}$ and would indicate a gas mass of $(1.0 \pm 0.3) \times 10^{6} M_{\odot}$, assuming the gas fills uniformly the bulge region, a sphere with $1 \mathrm{kpc}$ radius (using the power per unit emission integral as a function of temperature for a low density plasma reported by Kato 1976). Because a luminosity function derived from the detected sources in the heavily confused bulge region would be very uncertain, we cannot trust any estimation of the emission from non-detected sources below our detection threshold by extrapolating such a luminosity function. As a consequence, the above derived luminosity (and gas mass) of the diffuse emission must be considered as an upper limit.

Considering the disk, a subtraction of the background count rate and a multiplication with the disk area of $2.6 \mathrm{deg}^{2}$ yields $(1.68 \pm 0.14) \mathrm{ct} \mathrm{s}^{-1}$. A summation of the count rates of all the sources detected in the disk within the second PSPC survey data yields $(2.06 \pm 0.31) \mathrm{ct} \mathrm{s}^{-1}$. Here no correction had to be applied, as no important source confusion exists. This value is slightly higher than the one derived from the total emission. It may indicate a possible diffuse absorption of background photons by M 31. Although both derived count rates are comparable within their $1 \sigma$ errors, this is an effect of the integral consideration of the whole disk. A division into several annular regions indicates an absorption at the $1 \sigma$ significance level in some of these regions. A more detailed report will be the subject of a future paper. In the following discussion, we neglect a possible (slight) absorption in the M 31 disk.

As already mentioned in Sect. 4.2, a fair number of the detected sources do not belong to M 31, but are foreground sources or background sources shining through the galaxy. Therefore, the derived flux of all the resolved disk sources mentioned above (or the sum of the flux in the disk area) cannot be used for a determination of the total X-ray luminosity of the disk of M 31. Following the procedure described in S97 we use the there derived $\log N-\log S$ distribution for sources truly belonging to M 31 (from a statistical point of view). We come up with $(1.26 \pm 0.20) \mathrm{ct} \mathrm{s}^{-1}$ for the resulting count rate, or a total flux of $(1.7 \pm 0.3) \times 10^{-11} \mathrm{erg} \mathrm{cm}^{-2} \mathrm{~s}^{-1}$ for the disk of M 31 (using the above spectral model). This corresponds to a total luminosity of $(1.8 \pm 0.3) \times 10^{39} \mathrm{erg} \mathrm{s}^{-1}$.

All together, applying a power law spectral model with photon index $\Gamma=-2.0$ and a galactic foreground absorption of $N_{\mathrm{H}}=6 \times 10^{20} \mathrm{~cm}^{-2}$, we obtain for the total $(0.1-2.0 \mathrm{keV})$ luminosity of M 31, $(3.4 \pm 0.3) \times$ $10^{39} \mathrm{erg} \mathrm{s}^{-1}$, approximately equally distributed between the bulge and disk.

\subsection{Comparison with earlier results}

A comparison with the results derived from the first ROSAT PSPC survey of M 31 (S97) uncovered a difference in the bulge luminosities. For the total emission as well as for the sum of the resolved flux of detected sources we determined slightly higher values from the second PSPC survey data. Although the difference in significance for the total emission is less than $1.5 \sigma$, we decided to take the new value from the second survey as the better one due to the above mentioned reasons. Because the flux of the resolved emission increased approximately by the same (small) amount we would obtain nearly the same value for a possible gaseous component in the bulge of M 31 as previously derived from the first survey data when applying the same spectral model as used in $\mathrm{S} 97$ (now $(4.4 \pm 1.2) \times 10^{-12} \mathrm{erg} \mathrm{cm}^{-2} \mathrm{~s}^{-1}$, compared to $(4.6 \pm 1.1) \times 10^{-12} \mathrm{erg} \mathrm{cm}^{-2} \mathrm{~s}^{-1}$ in S97). It shows, that the change of the here newly given value $\left((3.4 \pm 0.9) \times 10^{-12} \mathrm{erg} \mathrm{cm}^{-2} \mathrm{~s}^{-1}\right)$ is mainly due to the new spectral model used (optically-thin thermal plasma with $k T=0.35 \mathrm{keV}$ ), which we adopted from recent results of XMM-Newton observations (Shirey et al. 2001). With this, the ROSAT derived diffuse luminosity within $5^{\prime}$ of the nucleus of M 31 is comparable to the luminosity found for the same bulge area with the Chandra (Garcia et al. 2000) and the XMM-Newton observation (Shirey et al. 2001). It is commonly assumed that the hot component of the interstellar medium (ISM) is created by winds from massive young stars and supernova explosions in star-forming 
regions. The diffuse emission from the hot ISM in M 31 is less pronounced than that detected from the inner spiral arms in the neighboring Local Group galaxy M 33. For this galaxy ROSAT HRI (Shulman \& Bregman 1994) and PSPC observations (Long et al. 1996) show diffuse emission with a luminosity of about $10^{39} \mathrm{erg} \mathrm{s}^{-1}$ that traces the spiral arms within $15^{\prime}$ of the nucleus and has a temperature of $k T=0.4 \mathrm{keV}$. Galaxies with high star-forming activity may be even brighter in diffuse X-rays by factors of more than 10 (see e.g. Read et al. 1997; Vogler \& Pietsch 1999a). The low diffuse X-ray luminosity in M 31 therefore supports the view that the galaxy is in a phase of low star-forming activity.

For the determination of the disk luminosity we adopted the procedure from our previous calculations used in the first survey. Hence, we obtained the same results. Also the considerations concerning the normalized luminosity distribution of the discrete $\mathrm{X}$-ray sources in the disk of M 31 are still valid (see S97). A comparison with the luminosity distributions (normalized to bulge luminosity) of other nearby spiral galaxies like M 33, M 51, M 83, M 100, M 101, NGC 253, NGC 1566, NGC 4258, NGC 4559, NGC 4565, and NGC 4631 (see Vogler \& Pietsch 1999b) shows no significant differencies in shape and reveals the distribution of M 31 as being typical for this class of galaxy. However, we do not find super-luminous sources (SLS) above several times $10^{38} \mathrm{erg} \mathrm{s}^{-1}$, as is also the case in M 33 and NGC 253, but not for the other (star-forming) galaxies mentioned above. Although NGC 253 is a (bulge) star-forming galaxy it shows no SLSs in its disk population. Therefore it is difficult to interpret the absence of SLSs in M 31, but it perhaps tends to show that M 31 is not in a star-forming phase.

The discussion of the comparison of our results with those obtained from the Einstein observatory and reported by $\mathrm{TF}$ also changes slightly under the transition from the first to the second PSPC survey. For the total luminosity of M 31, TF found a value of $\sim 3 \times 10^{39} \mathrm{erg} \mathrm{s}^{-1}$. To compare with our values, one has to take into account the different spectral models, energy ranges, and especially the different fields of M 31 investigated. TF derived the luminosities from the Einstein data by applying a thermal bremsstrahlung spectrum in the energy band $0.2 \mathrm{keV}-$ $4.0 \mathrm{keV}$ with $k T=5 \mathrm{keV}$ and $N_{\mathrm{H}}=7 \times 10^{20} \mathrm{~cm}^{-2}$. They integrated the count rates within an ellipse of $\sim 2.5^{\circ} \times 1.0^{\circ}$ which is a bit smaller than the $D_{25}$ ellipse we used for our calculations. A conversion of our results to the spectral model and reduced area of TF yields for the total luminosity $(3.3 \pm 0.3) \times 10^{39} \mathrm{erg} \mathrm{s}^{-1}$. The $1 \sigma$ agreement with the value reported by TF, however, is somewhat coincidental: while our observations covered the whole galaxy, those of TF did not. On the other hand, TF did not correct for background sources.

Comparing the total luminosity of the bulge region, $\mathrm{TF}$ reported $\sim 1.5 \times 10^{39} \mathrm{erg} \mathrm{s}^{-1}$, which is in agreement with our value of $1.6 \times 10^{39} \mathrm{erg} \mathrm{s}^{-1}$ (in this case the effect of the different assumed spectral models is below the errors and therefore negligible). In contrast, for the disk alone we found a somewhat higher luminosity $\left((1.8 \pm 0.3) \times 10^{39} \mathrm{erg} \mathrm{s}^{-1}\right)$ than TF $\left(\sim 1.5 \times 10^{39} \mathrm{erg} \mathrm{s}^{-1}\right)$, though there is still a $1 \sigma$ agreement. Considering the fact that $\mathrm{TF}$ did not describe the errors and furthermore did not explicitly quote the values for the bulge and disk emission, but simply mentioned that "the emission is roughly equally divided between the bulge and the disk", as well as their neglecting to compensate for background/foreground sources, we desist from a more quantitative comparison, noting that the agreement is surprisingly good. Our results tend to show that TF determined the disk luminosity too low and with it, the total luminosity of M 31. With the improved capabilities of ROSAT, the complete coverage of the total galaxy, and our considerations of statistical errors, we were able to clarify the luminosities in M 31 at a more reliable level.

As already mentioned, the second survey data did not (significantly) change the results concerning a possible diffuse emission component in the bulge region (from $(2.6 \pm 0.6) \times 10^{38} \mathrm{erg} \mathrm{s}^{-1}$ to $(2.5 \pm 0.7) \times 10^{38} \mathrm{erg} \mathrm{s}^{-1}$, when using the spectral model of S97). The exhaustive discussion of the comparison with the value reported by $\mathrm{TF}\left(\sim 3.8 \times 10^{38} \mathrm{erg} \mathrm{s}^{-1}\right)$ and the reasons for the difference have already been undertaken in S97, and are still valid.

\section{Summary and conclusions}

The second pointed ROSAT PSPC survey of M 31 has extended our knowledge concerning the $\mathrm{X}$-ray nature of this spiral galaxy beyond that already derived from the first survey described in S97 (Supper et al. 1997). Merging the two point source lists of the two surveys led to a total of 560 X-ray sources in the $\sim 10.7 \mathrm{deg}^{2} \mathrm{M} 31 \mathrm{FOV}, 31$ located in the very confused bulge region. Their luminosities range from $4 \times 10^{35} \mathrm{erg} \mathrm{s}^{-1}$ to $4 \times 10^{38} \mathrm{erg} \mathrm{s}^{-1}$, assuming a distance of $690 \mathrm{kpc}$ to M 31. Of these sources, 55 have been identified with known foreground stars, 33 with globular clusters, 16 with supernova remnants, and 10 correlate with known background objects such as background galaxies. None of our M 31 sources could be assigned to known novae. A comparison with the Einstein source list reported by TF confirms 69 Einstein sources. The much improved homogeneity of the second PSPC survey compared with the first and the resulting fewer problems with the PSPC support structure, allowed better flux determinations for a couple of sources. Combined with the higher positional precision in some regions, the list of variable sources when compared with the reported Einstein source fluxes could be restricted to 11 candidates, and 7 transients were discovered. Comparisons of the Einstein source list with the two ROSAT survey source lists separately, may yield up to 10 transients. Finally, of the 60 sources reported by $\mathrm{TF}$ outside the heavily confused bulge region, we could confirm 54, or $90 \%$ of these sources. In total, 39 Einstein sources could not be confirmed, while 491 new sources were found with ROSAT.

Comparing the first and second PSPC surveys of M 31, 34 possible long term variable sources and 8 possible 
transients (with some overlap with the transients obtained from the comparison with the Einstein detected sources) are reported.

For the bulge region, we can give an upper limit to the diffuse component luminosity of $(2.0 \pm 0.5) \times 10^{38} \mathrm{erg} \mathrm{s}^{-1}$ when using an optically-thin thermal plasma (MEKAL) with $k T=0.35 \mathrm{keV}$ for the spectral model. This is a factor of $\sim 1.5$ lower than the value reported by TF (after transforming to their spectral model). If we assume this luminosity as completely originating from hot gas within the bulge region, this would indicate a gas mass upper limit of $(1.0 \pm 0.3) \times 10^{6} M_{\odot}$. For the total $(0.1-2.0 \mathrm{keV})$ luminosity of M 31, we obtain $(3.4 \pm 0.3) \times 10^{39} \mathrm{erg} \mathrm{s}^{-1}$, for the bulge alone $1.6 \times 10^{39} \mathrm{erg} \mathrm{s}^{-1}$ and for the disk $(1.8 \pm 0.3) \times 10^{39} \mathrm{erg} \mathrm{s}^{-1}$. With these improved values, we find an equal distribution of luminosity between the bulge and disk, in agreement with TF, but a higher value for the total luminosity than reported in TF.

Several results from the first PSPC survey of M 31 reported in $\mathrm{S} 97$ have not been significantly altered by the inclusion of the second survey data and remain valid. These include: 1) the integral luminosity distribution of the globular cluster sources and its comparison to that of the Milky Way, 2) the statistical estimation of the fraction of background and foreground sources among the detected $\mathrm{X}$-ray sources, and 3) the spectral analysis of the brightest sources.

Acknowledgements. We thank the MPE ROSAT group for their support. Parts of our analysis used the SASS and EXSAS data analysis software. The authors would like to thank the referee for carefully reading this paper and making useful comments and suggestions. We thank NASA and the Centre de Données astronomiques for the online access to their data bases. WHGL is grateful for support from NASA.

RS remembers J. v. Paradijs as an always gentle and helpful excellent scientist who sadly passed away well ahead of his time - a great loss for the astronomical community.

The ROSAT project has been supported by the Bundesministerium für Bildung und Forschung (BMBF/DLR) and the Max-Planck-Gesellschaft (MPG).

\section{References}

Battistini, P. L., Bònoli, F., Braccesi, A., et al. 1987, A\&AS, 67, 447

Battistini, P. L., Bònoli, F., Casavecchia, M., et al. 1993, A\&A, 272,77

Borozdin, K. N., \& Priedhorsky, W. C. 2000, ApJ, 542, L13

Braun, R., \& Walterbos, R. A. M. 1993, A\&AS, 98, 327

Capaccioli, M., Della Valle, M., D'Onofrio, M., et al. 1989, AJ, 97, 1622

Collura, A., Reale, F., \& Peres, G. 1990, ApJ, 356, 119

Crampton, D., Cowley, A. P., Hutchings, J. B., Schade, D. J., \& van Speybroeck, L. P. 1984, ApJ, 284, 663

Cruddace, R. G., Hasinger, G. R., \& Schmitt, J. H. 1988, The Application of a Maximum Likelihood Analysis to Detection of Sources in the Rosat Data base, ed. F. Murtagh, \& A. Heck, Astronomy from Large Databases, ESO Conf. and Workshop Proc., No. 28
(Garching/Germany), 177

D’Odorico, S., Dopita, M. A., \& Benevenuti, P. 1980, A\&AS, 40,67

Garcia, M. R., Murray, S. S., Primini, F. A., et al. 2000, ApJ, 537, L23

Greiner, J., Supper, R., \& Magnier, E. A. 1996, Supersoft Xray Sources in M 31., in Supersoft X-ray Sources, ed. J. Greiner (Springer Verlag), 75

Haiman, Z, Magnier, E. A., Lewin, W. H. G., et al. 1994, A\&A, 286,725

Hasinger, G., et al. 1992, GSFC OGIP Calibration Memo, CAL/ROS/92-001

Immler, S. 2000, The X-Ray Source Population of Nearby Spiral Galaxies, Ph.D. Thesis, Ludwig-MaximiliansUniversität München, Germany

Irwin, J. A., \& Bregman, J. N. 1999, ApJ, 527, 125

Jacchia, L. G. 1972, in CIRA 1972: COSPAR International Reference Atmosphere 1972, compiled by The Committee for CIRA of Cospar Working Group 4 (Akademie-Verlag, Berlin), 227

Kahabka, P. 1999, A\&A, 344, 459

Kato, T. 1976, ApJS, 30, 397

Long, K. S., van Speybroeck, L. P. 1983, X-Ray Emission from Normal Galaxies, in Accretion Driven Stellar X-Ray Sources, ed. W. H. G. Lewin, \& E. P. J. van den Heuvel (Cambridge University Press Cambridge), 117

Long, K. S., Charles, P. A., Blair, W. P., et al. 1996, ApJ, 466, $750 \mathrm{~L}$

Magnier, E. A., Lewin, W. H. G., van Paradijs, J., et al. 1992, A\&AS, 96, 379

Magnier, E. A., Lewin, W. H. G., van Paradijs, J., et al. 1994a, A\&AS, submitted

Magnier, E. A., Prins, S., van Paradijs, J., et al. 1995, A\&AS, 114,215

Primini, F. A., Forman, W., \& Jones, C. 1993, ApJ, 410, 615

Read, A. M., Ponman, T. J., \& Stickland, D. K. 1997, MNRAS, 286,626

Sharov, A. S., \& Alksnis, A. 1991, Ap\&SS, 180, 273

Sharov, A. S., \& Alksnis, A. 1992, Ap\&SS, 190, 119

Shirey, R., Soria, R., Borozdin, K., et al. 2001, A\&A, 365, L195

Shulman, E., \& Bregman, J. N. 1994, ROSAT HRI Observations of M 33, ed. E. M. \& Schlegel, P. Robert, The Soft X-ray Cosmos, AIP Conf. Proc. \#313 (AIP, New York), 345

Snowden, S. L., Plucinsky, P. P., Briel, U., Hasinger, G., \& Pfeffermann, E. 1992, ApJ, 393, 819

Snowden, S. L., \& Freyberg, M. J. 1993, ApJ, 404, 403

van Speybroeck, L. P., Epstein, A., Forman, W., et al. 1979, ApJ, 234, L45

van Speybroeck, L. P., \& Bechtold, J. 1981, X-Ray Emission from Normal Galaxies, in X-Ray Astronomy with the Einstein Satellite, ed. R. Giacconi (Reidel, Dordrecht), 153

Supper, R., Hasinger, G., Pietsch, W., et al. 1997, A\&A, 317, 328 [S97]

Trinchieri, G., \& Fabbiano, G. 1991, ApJ, 382, 82 [TF]

Trinchieri, G., Israel, G. L., Chippetti, L., et al. 1999, A\&A, 348,43

Vogler, A., \& Pietsch, W. 1999a, A\&A, 342, 101

Vogler, A., \& Pietsch, W. 1999b, A\&A, 352, 64

Zimmermann, H. U., Belloni, T., Izzo, C., Kahabka, P., \& Schwentker, O. 1993, MPE Report 244, Ed. 3 
Table 4. Log of the 94 single observations forming the second ROSAT PSPC survey of M 31. Pointing numbers ending with " -1 " or " -2 " indicate follow-up observations.

\begin{tabular}{|c|c|c|c|c|c|c|c|c|c|}
\hline \multirow[t]{2}{*}{ Pointing } & \multirow[t]{2}{*}{ Date } & & \multicolumn{3}{|c|}{ RA $(J 2000)^{1}$} & \multicolumn{3}{|c|}{$\operatorname{Dec}(\mathrm{J} 2000)^{1}$} & \multirow{2}{*}{$\begin{array}{r}\text { Exposure } \\
(\mathrm{s})\end{array}$} \\
\hline & & & $(\mathrm{h})$ & $(\mathrm{m})$ & $\overline{(s)}$ & $\left({ }^{\circ}\right)$ & $\left({ }^{\prime}\right)$ & $\left({ }^{\prime \prime}\right)$ & \\
\hline WG600296P & 25.-26. July & 1992 & 0 & 37 & 43.2 & 40 & 23 & 24 & 2512 \\
\hline WG600297P & 25.-25. July & 1992 & 0 & 38 & 9.6 & 40 & 29 & 24 & 2536 \\
\hline WG600316P & 21.-21. July & 1992 & 0 & 38 & 26.3 & 40 & 17 & 24 & 2672 \\
\hline WG600298P & 05.-05. Aug. & 1992 & 0 & 38 & 33.5 & 40 & 36 & 00 & 2640 \\
\hline WG600317P & 20.-20. July & 1992 & 0 & 38 & 52.7 & 40 & 23 & 24 & 2616 \\
\hline WG600299P & 05.-05. Aug. & 1992 & 0 & 39 & 0.0 & 40 & 42 & 0 & 1576 \\
\hline WG600299P-1 & 01.-11. Jan. & 1993 & 0 & 39 & 0.0 & 40 & 42 & 0 & 1448 \\
\hline WG600336P & 29.-30. July & 1992 & 0 & 39 & 12.0 & 40 & 11 & 24 & 2752 \\
\hline WG600318P & 06.-06. Aug. & 1992 & 0 & 39 & 16.7 & 40 & 30 & 0 & 1416 \\
\hline WG600318P-1 & 31.-31. Dec. & 1992 & 0 & 39 & 16.7 & 40 & 30 & 0 & 1312 \\
\hline WG600300P & 05.-05. Aug. & 1992 & 0 & 39 & 24.0 & 40 & 48 & 36 & 2456 \\
\hline WG600337P & 30.-30. Dec. & 1992 & 0 & 39 & 36.0 & 40 & 17 & 24 & 2096 \\
\hline WG600319P & 06.-06. Aug. & 1992 & 0 & 39 & 43.2 & 40 & 36 & 00 & 1816 \\
\hline WG600301P & 22.-22. July & 1992 & 0 & 39 & 48.0 & 40 & 54 & 36 & 2688 \\
\hline WG600356P & 03.-04. Aug. & 1992 & 0 & 39 & 55.2 & 40 & 5 & 24 & 1976 \\
\hline WG600338P & 03.-03. Aug. & 1992 & 0 & 40 & 2.4 & 40 & 24 & 0 & 2168 \\
\hline WG600320P-1 & 01.-01. July & 1993 & 0 & 40 & 7.2 & 40 & 42 & 36 & 2744 \\
\hline WG600302P & 30.-31. Dec. & 1992 & 0 & 40 & 14.3 & 41 & 1 & 12 & 2568 \\
\hline WG600357P & 30.-30. Dec. & 1992 & 0 & 40 & 21.6 & 40 & 11 & 24 & 2720 \\
\hline WG600339P & 25.-25. July & 1992 & 0 & 40 & 26.3 & 40 & 30 & 0 & 2840 \\
\hline WG600321P & 29.-30. July & 1992 & 0 & 40 & 33.5 & 40 & 48 & 36 & 2848 \\
\hline WG600303P & 27.-28. July & 1992 & 0 & 40 & 38.4 & 41 & 7 & 12 & 2416 \\
\hline WG600358P & 26.-26. July & 1992 & 0 & 40 & 45.5 & 40 & 18 & 00 & 2864 \\
\hline WG600340P & 06.-06. Aug. & 1992 & 0 & 40 & 52.7 & 40 & 36 & 36 & 2560 \\
\hline WG600322P & 06.-06. Aug. & 1992 & 0 & 40 & 57.5 & 40 & 55 & 12 & 2584 \\
\hline WG600304P & 31.-31. Dec. & 1992 & 0 & 41 & 4.8 & 41 & 13 & 48 & 1872 \\
\hline WG600359P & 06.-07. Aug. & 1992 & 0 & 41 & 12.0 & 40 & 24 & 0 & 2600 \\
\hline WG600341P & 23.-23. July & 1992 & 0 & 41 & 16.7 & 40 & 42 & 36 & 2712 \\
\hline WG600323P & 05.-06. Aug. & 1992 & 0 & 41 & 24.0 & 41 & 1 & 12 & 2560 \\
\hline WG600305P & 06.-07. Aug. & 1992 & 0 & 41 & 28.7 & 41 & 19 & 48 & 2216 \\
\hline WG600360P & 23.-24. July & 1992 & 0 & 41 & 36.0 & 40 & 30 & 36 & 2864 \\
\hline WG600342P & 05.-05. Aug. & 1992 & 0 & 41 & 43.2 & 40 & 49 & 12 & 3416 \\
\hline WG600324P & 07.-07. Jan. & 1993 & 0 & 41 & 48.0 & 41 & 7 & 48 & 2960 \\
\hline WG600306P & 07.-08. Aug. & 1992 & 0 & 41 & 55.2 & 41 & 26 & 24 & 2424 \\
\hline WG600361P & 05.-06. Aug. & 1992 & 0 & 42 & 0.0 & 40 & 36 & 36 & 2744 \\
\hline WG600343P & 04.-04. Jan. & 1993 & 0 & 42 & 7.1 & 40 & 55 & 12 & 2744 \\
\hline WG600325P & 02.-02. Jan. & 1993 & 0 & 42 & 14.3 & 41 & 13 & 48 & 2848 \\
\hline WG600307P & 01.-01. Jan. & 1993 & 0 & 42 & 19.2 & 41 & 32 & 24 & 720 \\
\hline WG600307P-1 & 05.-09. July & 1993 & 0 & 42 & 19.2 & 41 & 32 & 24 & 2032 \\
\hline WG600362P & 06.-06. Aug. & 1992 & 0 & 42 & 26.3 & 40 & 43 & 12 & 2448 \\
\hline WG600344P & 07.-07. Aug. & 1992 & 0 & 42 & 31.2 & 41 & 1 & 48 & 1744 \\
\hline WG600326P & 07.-07. Aug. & 1992 & 0 & 42 & 38.4 & 41 & 20 & 24 & 1704 \\
\hline WG600326P-2 & 18.-18. July & 1993 & 0 & 42 & 38.4 & 41 & 20 & 24 & 696 \\
\hline WG600308P & 07.-07. Aug. & 1992 & 0 & 42 & 45.5 & 41 & 39 & 0 & 1512 \\
\hline WG600308P-1 & 03.-11. Jan. & 1993 & 0 & 42 & 45.5 & 41 & 39 & 0 & 1768 \\
\hline WG600363P & 03.-03. Jan. & 1993 & 0 & 42 & 50.4 & 40 & 49 & 12 & 1944 \\
\hline WG600345P & 01.-01. Jan. & 1993 & 0 & 42 & 57.5 & 41 & 7 & 48 & 1592 \\
\hline WG600345P-1 & 22.-22. July & 1993 & 0 & 42 & 57.6 & 41 & 7 & 48 & 1712 \\
\hline
\end{tabular}


Table 4. continued.

\begin{tabular}{|c|c|c|c|c|c|c|c|c|c|}
\hline \multirow[t]{2}{*}{ Pointing } & \multirow[t]{2}{*}{ Date } & & \multicolumn{3}{|c|}{$\mathrm{RA}(\mathrm{J} 2000)^{1}$} & \multicolumn{3}{|c|}{$\operatorname{Dec}(\mathrm{J} 2000)^{1}$} & \multirow{2}{*}{$\begin{array}{r}\text { Exposure } \\
(\mathrm{s}) \\
\end{array}$} \\
\hline & & & (h) & $(\mathrm{m})$ & $(\mathrm{s})$ & $\left({ }^{\circ}\right)$ & $\left(^{\prime}\right)$ & $\left({ }^{\prime \prime}\right)$ & \\
\hline WG600327P & 07.-07. Aug. & 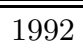 & 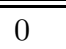 & 43 & 4.8 & 41 & 26 & 24 & 2112 \\
\hline WG600309P & 07.-08. Aug. & 1992 & 0 & 43 & 9.6 & 41 & 45 & 0 & 2576 \\
\hline WG600364P & 07.-07. Aug. & 1992 & 0 & 43 & 16.7 & 40 & 55 & 48 & 416 \\
\hline WG600364P-2 & 04.-04. July & 1993 & 0 & 43 & 16.8 & 40 & 55 & 48 & 3296 \\
\hline WG600346P & 06.-06. Aug. & 1992 & 0 & 43 & 21.6 & 41 & 14 & 24 & 1968 \\
\hline WG600328P & 02.-02. Jan. & 1993 & 0 & 43 & 28.7 & 41 & 33 & 00 & 2320 \\
\hline WG600310P & 05.-05. Jan. & 1993 & 0 & 43 & 36.0 & 41 & 51 & 36 & 2720 \\
\hline WG600365P & 02.-02. Jan. & 1993 & 0 & 43 & 40.7 & 41 & 1 & 48 & 3144 \\
\hline WG600347P & 03.-03. Jan. & 1993 & 0 & 43 & 48.0 & 41 & 20 & 24 & 2936 \\
\hline WG600329P & 08.-08. Aug. & 1992 & 0 & 43 & 52.7 & 41 & 39 & 0 & 1760 \\
\hline WG600311P & 08.-08. Aug. & 1992 & 0 & 44 & 0.0 & 41 & 58 & 12 & 1352 \\
\hline WG600311P-1 & 01.-01. Jan. & 1993 & 0 & 44 & 0.0 & 41 & 58 & 12 & 1240 \\
\hline WG600366P & 07.-07. Aug. & 1992 & 0 & 44 & 7.1 & 41 & 8 & 24 & 1776 \\
\hline WG600348P & 31.-31. Dec. & 1992 & 0 & 44 & 12.0 & 41 & 27 & 0 & 1696 \\
\hline WG600348P-1 & 22.-22. July & 1993 & 0 & 44 & 12.0 & 41 & 27 & 0 & 1800 \\
\hline WG600330P & 08.-08. Aug. & 1992 & 0 & 44 & 19.2 & 41 & 45 & 36 & 1624 \\
\hline WG600330P-1 & 14.-14. Jan. & 1993 & 0 & 44 & 19.2 & 41 & 45 & 36 & 944 \\
\hline WG600312P & 08.-08. Aug. & 1992 & 0 & 44 & 24.0 & 42 & 4 & 12 & 2416 \\
\hline WG600367P & 04.-04. Jan. & 1993 & 0 & 44 & 31.2 & 41 & 14 & 24 & 2624 \\
\hline WG600349P & 06.-06. Jan. & 1993 & 0 & 44 & 38.4 & 41 & 33 & 00 & 2824 \\
\hline WG600331P & 04.-04. Jan. & 1993 & 0 & 44 & 43.2 & 41 & 52 & 12 & 2872 \\
\hline WG600313P & 09.-09. Jan. & 1993 & 0 & 44 & 50.4 & 42 & 10 & 48 & 2672 \\
\hline WG600368P & 03.-03. Jan. & 1993 & 0 & 44 & 57.5 & 41 & 21 & 00 & 2912 \\
\hline WG600350P & 05.-05. Jan. & 1993 & 0 & 45 & 2.4 & 41 & 39 & 36 & 2680 \\
\hline WG600332P & 11.-11. Jan. & 1993 & 0 & 45 & 9.6 & 41 & 58 & 12 & 2944 \\
\hline WG600314P & 10.-10. Jan. & 1993 & 0 & 45 & 14.3 & 42 & 16 & 48 & 2704 \\
\hline WG600369P & 09.-09. Jan. & 1993 & 0 & 45 & 21.6 & 41 & 27 & 0 & 2520 \\
\hline WG600351P & 02.-02. Jan. & 1993 & 0 & 45 & 28.7 & 41 & 46 & 12 & 2824 \\
\hline WG600333P & 11.-11. Jan. & 1993 & 0 & 45 & 33.5 & 42 & 4 & 48 & 3040 \\
\hline WG600315P & 06.-10. Jan. & 1993 & 0 & 45 & 40.7 & 42 & 23 & 24 & 3024 \\
\hline WG600370P & 06.-06. Jan. & 1993 & 0 & 45 & 48.0 & 41 & 33 & 36 & 2864 \\
\hline WG600352P & 10.-10. Jan. & 1993 & 0 & 45 & 52.7 & 41 & 52 & 12 & 2632 \\
\hline WG600334P & 09.-09. Aug. & 1992 & 0 & 46 & 0.0 & 42 & 10 & 48 & 720 \\
\hline WG600334P-1 & 07.-07. Jan. & 1993 & 0 & 46 & 0.0 & 42 & 10 & 48 & 2168 \\
\hline WG600371P & 08.-09. Aug. & 1992 & 0 & 46 & 12.0 & 41 & 40 & 12 & 2080 \\
\hline WG600353P & 11.-11. Jan. & 1993 & 0 & 46 & 19.2 & 41 & 58 & 48 & 3008 \\
\hline WG600335P & 09.-09. Aug. & 1992 & 0 & 46 & 24.0 & 42 & 17 & 24 & 728 \\
\hline WG600335P-1 & 10.-10. Jan. & 1993 & 0 & 46 & 24.0 & 42 & 17 & 24 & 2368 \\
\hline WG600372P & 03.-03. July & 1993 & 0 & 46 & 38.4 & 41 & 46 & 12 & 3088 \\
\hline WG600354P & 08.-08. Aug. & 1992 & 0 & 46 & 43.2 & 42 & 4 & 48 & 2368 \\
\hline WG600373P & 11.-12. Jan. & 1993 & 0 & 47 & 2.4 & 41 & 52 & 48 & 3144 \\
\hline WG600355P & 09.-09. Aug. & 1992 & 0 & 47 & 9.6 & 42 & 11 & 24 & 1920 \\
\hline WG600374P & 09.-09. Aug. & 1992 & 0 & 47 & 26.3 & 41 & 58 & 48 & 680 \\
\hline WG600374P-1 & 04.-04. Jan. & 1993 & 0 & 47 & 26.3 & 41 & 58 & 48 & 2008 \\
\hline WG600375P & 09.-09. Aug. & 1992 & 0 & 47 & 52.7 & 42 & 5 & 24 & 1080 \\
\hline WG600375P-1 & 15.-15. Jan. & 1993 & 0 & 47 & 52.7 & 42 & 5 & 24 & 2112 \\
\hline
\end{tabular}

\footnotetext{
${ }^{1}$ The coordinates give the centre of the FOV (nominal pointing direction).
} 
Table 5. List of all X-ray sources in M 31 detected in the second ROSAT PSPC survey $(S I I)$. The meaning of the different columns is described in Sect. 3.3. The listed count rate errors are only statistical. The systematic errors are expected to be less than $\sim 15 \%$. For sources not detected in a considered energy band $1 \sigma$ upper limits have been calculated indicated by a "<"-symbol in front of the upper limit value. A conversion of count rates into fluxes depends on the assumed spectral shape. For M 31-sources a power law with $\Gamma=-2.0$ and $N_{\mathrm{H}}=9 \times 10^{20} \mathrm{~cm}^{-2}$ may be used, leading to the conversion factor $1 \mathrm{cts} \mathrm{ksec}{ }^{-1}=3.00 \times 10^{-14} \mathrm{erg} \mathrm{cm}^{-2} \mathrm{~s}^{-1}$ in the $0.1-2.0 \mathrm{keV}$ band (B-band). For foreground stars the application of this conversion factor leads to an over-estimate of the fluxes.

\begin{tabular}{|c|c|c|c|c|c|c|c|c|c|c|c|c|c|c|}
\hline $\begin{array}{l}S I I \\
\text { No. } \\
(1)\end{array}$ & $\begin{array}{l}{ }^{\mathrm{I}} \\
(\mathrm{h}) \\
(2)\end{array}$ & $\begin{array}{l}\text { A } \\
(\mathrm{m}) \\
(3)\end{array}$ & $\begin{array}{l}(\mathrm{J} 20 \\
(\mathrm{s}) \\
(4)\end{array}$ & $\begin{array}{l}00) \\
\left(\begin{array}{l}0 \\
(5)\end{array}\right. \\
(5)\end{array}$ & $\begin{aligned} & \mathrm{D} \\
&\left({ }^{\prime}\right) \\
&(6)\end{aligned}$ & $\begin{array}{l}\mathrm{c} \\
\left({ }^{\prime \prime}\right) \\
(7)\end{array}$ & $\begin{array}{r}\sigma_{\text {Pos }} \\
\left({ }^{\prime \prime}\right) \\
(8)\end{array}$ & $\begin{array}{l}\text { Cl. } \\
\text { (9) }\end{array}$ & $\begin{array}{c}\text { Maxlik } \\
(\mathrm{LH}) \\
(10)\end{array}$ & $\begin{array}{c}\text { Rate }(B) \\
\left(\mathrm{ct} \cdot \mathrm{ks}^{-1}\right) \\
(11)\end{array}$ & $\begin{array}{c}\text { Rate }(S) \\
\left(\mathrm{ct} \cdot \mathrm{ks}^{-1}\right) \\
(12)\end{array}$ & $\begin{array}{c}\text { Rate }(H) \\
\left(\mathrm{ct} \cdot \mathrm{ks}^{-1}\right) \\
(13)\end{array}$ & $\begin{array}{c}\operatorname{Rate}\left(H_{1}\right) \\
\left(\mathrm{ct} \cdot \mathrm{ks}^{-1}\right) \\
(14)\end{array}$ & $\begin{array}{c}\operatorname{Rate}\left(H_{2}\right) \\
\left(\mathrm{ct} \cdot \mathrm{ks}^{-1}\right) \\
(15)\end{array}$ \\
\hline 1 & 0 & 36 & 21.7 & 40 & 53 & 37 & 13 & 4 & 69.7 & $<0.61$ & $<0.20$ & $5.73 \pm 0.65$ & $<1.70$ & $4.22 \pm 0.53$ \\
\hline 2 & 0 & 36 & 43.0 & 41 & 9 & 2 & 19 & 4 & 27.1 & $<0.43$ & $<0.16$ & $3.18 \pm 0.71$ & $<0.87$ & $3.56 \pm 0.61$ \\
\hline 3 & 0 & 36 & 49.6 & 40 & 55 & 12 & 18 & 4 & 11.8 & $<0.13$ & $<0.14$ & $1.72 \pm 0.42$ & $<0.99$ & $<1.20$ \\
\hline 4 & 0 & 36 & 49.8 & 40 & 8 & 52 & 12 & 1 & 45.0 & $10.62 \pm 1.82$ & $<3.50$ & $7.46 \pm 1.43$ & $<3.59$ & $3.74 \pm 1.04$ \\
\hline 5 & 0 & 36 & 57.4 & 40 & 26 & 10 & 12 & 1 & 12.2 & $<2.13$ & $<0.62$ & $1.34 \pm 0.43$ & $<0.81$ & $<1.30$ \\
\hline 6 & 0 & 37 & 11.4 & 40 & 22 & 10 & 11 & 1 & 10.5 & $<1.65$ & $<0.70$ & $<1.13$ & $<0.63$ & $<0.55$ \\
\hline 7 & 0 & 37 & 18.7 & 40 & 29 & 47 & 8 & 1 & 82.4 & $3.43 \pm 0.65$ & $<0.30$ & $3.90 \pm 0.60$ & $2.02 \pm 0.43$ & $1.79 \pm 0.41$ \\
\hline 8 & 0 & 37 & 22.5 & 40 & 43 & 49 & 9 & 1 & 86.3 & $10.19 \pm 1.34$ & $5.09 \pm 0.98$ & $4.93 \pm 0.90$ & $2.97 \pm 0.69$ & $1.82 \pm 0.56$ \\
\hline 9 & 0 & 37 & 26.2 & 40 & 13 & 7 & 10 & 1 & 20.6 & $2.16 \pm 0.60$ & $<0.79$ & $1.63 \pm 0.43$ & $<0.54$ & $1.22 \pm 0.36$ \\
\hline 10 & 0 & 37 & 34.6 & 40 & 9 & 55 & 7 & 1 & 238.8 & $11.48 \pm 1.16$ & $<1.25$ & $9.49 \pm 0.97$ & $3.63 \pm 0.61$ & $5.63 \pm 0.75$ \\
\hline 11 & 0 & 37 & 38.5 & 39 & 38 & 42 & 16 & 4 & 81.2 & $<6.05$ & $<0.62$ & $10.39 \pm 1.17$ & $<3.21$ & $8.48 \pm 0.96$ \\
\hline 12 & 0 & 37 & 41.5 & 40 & 25 & 45 & 10 & 1 & 15.9 & $<1.62$ & $<0.59$ & $0.78 \pm 0.25$ & $<0.36$ & $0.51 \pm 0.19$ \\
\hline 13 & 0 & 37 & 42.6 & 40 & 37 & 50 & 10 & 1 & 15.9 & $<1.86$ & $<0.73$ & $0.90 \pm 0.28$ & $<0.45$ & $0.58 \pm 0.22$ \\
\hline 14 & 0 & 37 & 43.3 & 40 & 23 & 44 & 8 & 1 & 52.3 & $2.97 \pm 0.54$ & $<1.30$ & $2.12 \pm 0.39$ & $1.64 \pm 0.34$ & $<0.62$ \\
\hline 15 & 0 & 37 & 43.5 & 40 & 4 & 55 & 11 & 1 & 44.0 & $19.11 \pm 2.03$ & $<2.32$ & $14.48 \pm 1.49$ & $4.25 \pm 0.89$ & $8.80 \pm 1.15$ \\
\hline 16 & 0 & 38 & 0.5 & 40 & 26 & 34 & 6 & 1 & 578.9 & $16.59 \pm 0.99$ & $9.23 \pm 0.78$ & $7.05 \pm 0.61$ & $3.24 \pm 0.41$ & $3.86 \pm 0.45$ \\
\hline 17 & 0 & 38 & 20.1 & 40 & 40 & 29 & 10 & 1 & 17.2 & $<1.39$ & $<0.50$ & $0.75 \pm 0.23$ & $<0.22$ & $0.60 \pm 0.20$ \\
\hline 18 & 0 & 38 & 21.3 & 40 & 56 & 40 & 9 & 1 & 52.1 & $7.23 \pm 1.16$ & $3.23 \pm 0.92$ & $3.99 \pm 0.80$ & $1.42 \pm 0.50$ & $2.49 \pm 0.63$ \\
\hline 19 & 0 & 38 & 21.5 & 40 & 18 & 32 & 12 & 1 & 12.8 & $<0.92$ & $<0.33$ & $0.54 \pm 0.19$ & $<0.22$ & $0.52 \pm 0.18$ \\
\hline 20 & 0 & 38 & 23.5 & 40 & 12 & 59 & 7 & 1 & 327.8 & $9.03 \pm 0.78$ & $<1.67$ & $7.21 \pm 0.64$ & $3.08 \pm 0.42$ & $4.17 \pm 0.49$ \\
\hline 21 & 0 & 38 & 27.7 & 41 & 36 & 55 & 23 & 4 & 14.6 & $<0.17$ & $<0.17$ & $<2.86$ & $<0.46$ & $<0.68$ \\
\hline 22 & 0 & 38 & 34.5 & 41 & 28 & 51 & 8 & 4 & 2093.6 & $55.28 \pm 0.96$ & $<0.40$ & $63.69 \pm 1.42$ & $21.82 \pm 0.91$ & $35.02 \pm 1.17$ \\
\hline 23 & 0 & 38 & 34.8 & 40 & 10 & 34 & 10 & 1 & 22.9 & $<2.03$ & $<0.55$ & $1.32 \pm 0.32$ & $<0.51$ & $1.07 \pm 0.27$ \\
\hline 24 & 0 & 38 & 38.6 & 40 & 26 & 21 & 7 & 1 & 144.6 & $3.87 \pm 0.46$ & $<0.84$ & $2.85 \pm 0.35$ & $1.77 \pm 0.27$ & $1.06 \pm 0.22$ \\
\hline 25 & 0 & 38 & 38.6 & 40 & 56 & 54 & 11 & 1 & 26.0 & $2.59 \pm 0.71$ & $<0.48$ & $2.57 \pm 0.57$ & $<0.82$ & $2.25 \pm 0.53$ \\
\hline 26 & 0 & 38 & 39.0 & 40 & 43 & 25 & 10 & 1 & 11.7 & $<0.80$ & $<0.25$ & $<0.77$ & $<0.18$ & $0.44 \pm 0.17$ \\
\hline 27 & 0 & 38 & 40.0 & 40 & 20 & 5 & 11 & 1 & 19.4 & $1.52 \pm 0.36$ & $1.63 \pm 0.36$ & $<0.25$ & $<0.18$ & $<0.11$ \\
\hline 28 & 0 & 38 & 40.8 & 40 & 44 & 57 & 9 & 1 & 20.3 & $1.46 \pm 0.39$ & $<0.98$ & $0.78 \pm 0.24$ & $<0.18$ & $0.69 \pm 0.21$ \\
\hline 29 & 0 & 38 & 48.2 & 40 & 8 & 3 & 10 & 1 & 23.9 & $1.50 \pm 0.44$ & $<0.60$ & $1.39 \pm 0.33$ & $<1.20$ & $0.64 \pm 0.21$ \\
\hline 30 & 0 & 38 & 48.6 & 40 & 38 & 12 & 8 & 1 & 39.5 & $1.28 \pm 0.31$ & $<0.26$ & $1.18 \pm 0.24$ & $0.43 \pm 0.15$ & $0.73 \pm 0.19$ \\
\hline 31 & 0 & 38 & 50.1 & 40 & 44 & 18 & 8 & 1 & 111.0 & $3.28 \pm 0.49$ & $<0.45$ & $3.25 \pm 0.43$ & $<0.87$ & $2.70 \pm 0.38$ \\
\hline 32 & 0 & 38 & 56.0 & 39 & 55 & 51 & 10 & 1 & 26.2 & $2.52 \pm 0.74$ & $<0.66$ & $2.07 \pm 0.59$ & $<0.73$ & $1.57 \pm 0.50$ \\
\hline 33 & 0 & 38 & 56.3 & 40 & 34 & 52 & 9 & 1 & 43.3 & $1.69 \pm 0.34$ & $<0.50$ & $1.35 \pm 0.25$ & $<0.42$ & $1.03 \pm 0.21$ \\
\hline 34 & 0 & 39 & 3.6 & 40 & 47 & 43 & 9 & 1 & 10.9 & $<1.03$ & $<0.37$ & $0.59 \pm 0.20$ & $<0.30$ & $0.39 \pm 0.15$ \\
\hline 35 & 0 & 39 & 9.1 & 40 & 6 & 23 & 12 & 1 & 12.8 & $<0.85$ & $<0.36$ & $<0.87$ & $<0.19$ & $0.58 \pm 0.21$ \\
\hline 36 & 0 & 39 & 11.6 & 40 & 35 & 34 & 10 & 1 & 16.8 & $<1.11$ & $<0.30$ & $0.66 \pm 0.18$ & $0.51 \pm 0.16$ & $<0.23$ \\
\hline 37 & 0 & 39 & 12.8 & 40 & 13 & 8 & 10 & 1 & 14.9 & $<1.36$ & $<0.63$ & $0.56 \pm 0.19$ & $<0.20$ & $0.46 \pm 0.16$ \\
\hline 38 & 0 & 39 & 15.4 & 40 & 8 & 21 & 9 & 1 & 44.8 & $2.04 \pm 0.46$ & $<0.37$ & $2.10 \pm 0.38$ & $0.92 \pm 0.25$ & $1.24 \pm 0.28$ \\
\hline 39 & 0 & 39 & 16.5 & 40 & 1 & 6 & 9 & 1 & 25.0 & $2.21 \pm 0.61$ & $<2.02$ & $1.69 \pm 0.44$ & $<0.96$ & $1.53 \pm 0.42$ \\
\hline 40 & 0 & 39 & 16.6 & 40 & 51 & 17 & 8 & 1 & 35.4 & $1.64 \pm 0.38$ & $<0.42$ & $1.33 \pm 0.28$ & $<0.78$ & $0.84 \pm 0.21$ \\
\hline 41 & 0 & 39 & 17.9 & 41 & 3 & 0 & 10 & 1 & 33.8 & $1.62 \pm 0.47$ & $<0.26$ & $2.17 \pm 0.45$ & $<1.00$ & $1.40 \pm 0.36$ \\
\hline 42 & 0 & 39 & 25.0 & 41 & 5 & 20 & 11 & 1 & 12.3 & $<1.80$ & $<0.54$ & $0.98 \pm 0.32$ & $<0.47$ & $0.66 \pm 0.26$ \\
\hline 43 & 0 & 39 & 25.1 & 40 & 50 & 30 & 9 & 1 & 25.1 & $1.88 \pm 0.38$ & $<1.33$ & $0.81 \pm 0.21$ & $0.45 \pm 0.15$ & $<0.51$ \\
\hline 44 & 0 & 39 & 29.2 & 40 & 35 & 55 & 10 & 1 & 11.5 & $<1.09$ & $<0.43$ & $0.50 \pm 0.16$ & $<0.27$ & $<0.61$ \\
\hline 45 & 0 & 39 & 34.6 & 41 & 9 & 34 & 8 & 1 & 93.4 & $5.04 \pm 0.81$ & $<0.53$ & $4.87 \pm 0.72$ & $1.09 \pm 0.36$ & $3.75 \pm 0.63$ \\
\hline 46 & 0 & 39 & 38.0 & 40 & 48 & 3 & 9 & 1 & 18.7 & $1.02 \pm 0.30$ & $<1.18$ & $0.78 \pm 0.20$ & $<0.38$ & $0.54 \pm 0.16$ \\
\hline 47 & 0 & 39 & 38.3 & 40 & 11 & 6 & 9 & 1 & 26.2 & $1.86 \pm 0.41$ & $<0.93$ & $1.04 \pm 0.25$ & $0.65 \pm 0.20$ & $0.45 \pm 0.16$ \\
\hline 48 & 0 & 39 & 39.9 & 40 & 35 & 37 & 9 & 1 & 31.6 & $1.18 \pm 0.28$ & $<0.33$ & $1.00 \pm 0.21$ & $<0.50$ & $0.66 \pm 0.16$ \\
\hline 49 & 0 & 39 & 42.6 & 40 & 39 & 48 & 9 & 1 & 24.5 & $1.03 \pm 0.26$ & $<0.34$ & $0.79 \pm 0.18$ & $0.62 \pm 0.16$ & $<0.31$ \\
\hline 50 & 0 & 39 & 43.0 & 41 & 16 & 3 & 20 & 1 & 15.0 & $<11.52$ & $<0.87$ & $4.67 \pm 1.13$ & $<2.19$ & $3.11 \pm 0.87$ \\
\hline 51 & 0 & 39 & 47.5 & 40 & 30 & 17 & 9 & 1 & 28.4 & $1.89 \pm 0.34$ & $1.68 \pm 0.32$ & $<0.34$ & $<0.28$ & $<0.12$ \\
\hline 52 & 0 & 39 & 48.8 & 41 & 10 & 8 & 9 & 1 & 22.6 & $<2.03$ & $<0.35$ & $1.51 \pm 0.39$ & $<0.88$ & $0.88 \pm 0.29$ \\
\hline 53 & 0 & 39 & 53.8 & 40 & 9 & 1 & 7 & 1 & 85.1 & $3.03 \pm 0.49$ & $<0.58$ & $2.60 \pm 0.40$ & $1.17 \pm 0.28$ & $1.47 \pm 0.30$ \\
\hline 54 & 0 & 39 & 56.1 & 40 & 41 & 6 & 7 & 1 & 100.5 & $2.44 \pm 0.34$ & $<0.36$ & $1.91 \pm 0.26$ & $1.15 \pm 0.20$ & $0.76 \pm 0.17$ \\
\hline 55 & 0 & 39 & 56.4 & 41 & 11 & 39 & 10 & 1 & 14.0 & $<1.55$ & $<0.33$ & $1.15 \pm 0.36$ & $<1.27$ & $<0.53$ \\
\hline 56 & 0 & 39 & 57.5 & 40 & 27 & 31 & 8 & 1 & 57.6 & $2.08 \pm 0.35$ & $<0.44$ & $1.61 \pm 0.26$ & $0.84 \pm 0.20$ & $0.80 \pm 0.18$ \\
\hline 57 & 0 & 39 & 57.6 & 40 & 16 & 32 & 9 & 1 & 20.8 & $1.23 \pm 0.32$ & $<1.64$ & $0.74 \pm 0.19$ & $<0.38$ & $0.49 \pm 0.15$ \\
\hline 58 & 0 & 39 & 58.1 & 39 & 29 & 33 & 37 & 4 & 10.3 & $<0.70$ & $<0.55$ & $<3.01$ & $<1.01$ & $<3.21$ \\
\hline 59 & 0 & 39 & 59.2 & 40 & 32 & 8 & 8 & 1 & 104.0 & $2.97 \pm 0.39$ & $<0.68$ & $2.85 \pm 0.34$ & $0.74 \pm 0.18$ & $2.00 \pm 0.27$ \\
\hline 60 & 0 & 39 & 59.5 & 41 & 1 & 3 & 11 & 1 & 20.2 & $<2.13$ & $<0.27$ & $2.20 \pm 0.40$ & $1.35 \pm 0.31$ & $<1.13$ \\
\hline
\end{tabular}


Table 5. continued.

\begin{tabular}{|c|c|c|c|c|c|c|c|c|c|c|c|c|c|c|}
\hline $\begin{array}{l}S I I \\
\text { No. } \\
(1)\end{array}$ & $\begin{array}{l}\text { I } \\
\text { (h) } \\
(2)\end{array}$ & $\begin{array}{l}\text { A } \\
(\mathrm{m}) \\
(3)\end{array}$ & $\begin{array}{l}(\mathrm{J} 2 \mathrm{C} \\
(\mathrm{s}) \\
(4)\end{array}$ & $\begin{array}{l}00) \\
\left({ }^{\circ}\right) \\
(5)\end{array}$ & $\begin{array}{r}\text { D } \\
\left({ }^{\prime}\right) \\
(6)\end{array}$ & $\begin{array}{l}\text { ec } \\
\left({ }^{\prime \prime}\right) \\
(7)\end{array}$ & $\begin{array}{r}\sigma_{\text {Pos }} \\
\left({ }^{\prime \prime}\right) \\
(8)\end{array}$ & $\begin{array}{l}\mathrm{Cl} . \\
(9)\end{array}$ & $\begin{array}{c}\text { Maxlik } \\
(\mathrm{LH}) \\
(10)\end{array}$ & $\begin{array}{c}\text { Rate }(B) \\
\left(\mathrm{ct} \cdot \mathrm{ks}^{-1}\right) \\
(11)\end{array}$ & $\begin{array}{c}\text { Rate }(S) \\
\left(\mathrm{ct} \cdot \mathrm{ks}^{-1}\right) \\
(12)\end{array}$ & $\begin{array}{c}\text { Rate }(H) \\
\left(\mathrm{ct} \cdot \mathrm{ks}^{-1}\right) \\
(13)\end{array}$ & $\begin{array}{c}\text { Rate }\left(H_{1}\right) \\
\left(\mathrm{ct} \cdot \mathrm{ks}^{-1}\right) \\
(14)\end{array}$ & $\begin{array}{c}\text { Rate }\left(H_{2}\right) \\
\left(\mathrm{ct} \cdot \mathrm{ks}^{-1}\right) \\
(15)\end{array}$ \\
\hline 61 & 0 & 40 & 7.6 & 40 & 6 & 28 & 14 & 1 & 11.5 & $<2.23$ & $<1.24$ & $<1.27$ & $<0.18$ & $<1.34$ \\
\hline 62 & 0 & 40 & 7.6 & 40 & 53 & 3 & 12 & 1 & 10.2 & $<0.64$ & $<0.20$ & $0.70 \pm 0.21$ & $<0.47$ & $<0.50$ \\
\hline 63 & 0 & 40 & 11.3 & 40 & 59 & 30 & 9 & 1 & 16.7 & $<1.32$ & $<0.37$ & $0.78 \pm 0.22$ & $<0.40$ & $0.51 \pm 0.18$ \\
\hline 64 & 0 & 40 & 13.5 & 40 & 50 & 10 & 5 & 1 & 16287. & $118.14 \pm 1.78$ & $1.72 \pm 0.36$ & $124.12 \pm 1.94$ & $23.39 \pm 0.87$ & $103.45 \pm 1.86$ \\
\hline 65 & 0 & 40 & 13.9 & 40 & 33 & 52 & 8 & 1 & 59.8 & $1.84 \pm 0.32$ & $<0.26$ & $1.59 \pm 0.25$ & $0.81 \pm 0.19$ & $0.90 \pm 0.18$ \\
\hline 66 & 0 & 40 & 14.8 & 40 & 15 & 17 & 11 & 1 & 11.0 & $<1.06$ & $<0.36$ & $0.63 \pm 0.21$ & $<0.61$ & $<0.44$ \\
\hline 67 & 0 & 40 & 17.7 & 39 & 53 & 54 & 8 & 1 & 99.8 & $9.65 \pm 1.45$ & $<2.27$ & $7.97 \pm 1.26$ & $4.25 \pm 0.91$ & $3.67 \pm 0.87$ \\
\hline 68 & 0 & 40 & 19.9 & 40 & 44 & 5 & 5 & 1 & 6379.9 & $52.34 \pm 1.27$ & $1.39 \pm 0.28$ & $48.99 \pm 1.21$ & $14.45 \pm 0.65$ & $34.80 \pm 1.02$ \\
\hline 69 & 0 & 40 & 21.5 & 40 & 3 & 20 & 13 & 1 & 10.6 & $<1.87$ & $<0.38$ & $<1.90$ & $<0.28$ & $1.07 \pm 0.35$ \\
\hline 70 & 0 & 40 & 22.6 & 40 & 36 & 17 & 10 & 1 & 19.2 & $<1.01$ & $<0.24$ & $0.71 \pm 0.18$ & $<0.29$ & $0.54 \pm 0.14$ \\
\hline 71 & 0 & 40 & 22.9 & 40 & 53 & 15 & 9 & 1 & 26.9 & $1.61 \pm 0.32$ & $<0.73$ & $0.97 \pm 0.21$ & $0.59 \pm 0.17$ & $<0.51$ \\
\hline 72 & 0 & 40 & 23.7 & 40 & 29 & 53 & 7 & 1 & 187.0 & $4.21 \pm 0.45$ & $<0.56$ & $3.44 \pm 0.36$ & $0.93 \pm 0.20$ & $2.47 \pm 0.30$ \\
\hline 73 & 0 & 40 & 25.3 & 40 & 34 & 30 & 10 & 1 & 11.2 & $<0.82$ & $<0.35$ & $<0.60$ & $<0.19$ & $0.35 \pm 0.12$ \\
\hline 74 & 0 & 40 & 25.8 & 41 & 29 & 12 & 12 & 1 & 35.6 & $8.58 \pm 1.51$ & $<1.31$ & $7.79 \pm 1.28$ & $<1.64$ & $6.94 \pm 1.18$ \\
\hline 75 & 0 & 40 & 32.1 & 40 & 1 & 12 & 11 & 1 & 16.0 & $2.12 \pm 0.63$ & $<1.23$ & $1.49 \pm 0.46$ & $<1.14$ & $<0.99$ \\
\hline 76 & 0 & 40 & 32.4 & 40 & 33 & 28 & 16 & 1 & 12.4 & $<1.08$ & $<0.42$ & $<0.73$ & $<0.16$ & $<0.94$ \\
\hline 77 & 0 & 40 & 35.6 & 39 & 57 & 18 & 12 & 1 & 10.4 & $2.55 \pm 0.80$ & $<3.27$ & $<0.99$ & $<0.44$ & $<0.60$ \\
\hline 78 & 0 & 40 & 38.1 & 41 & 19 & 39 & 14 & 1 & 11.1 & $<3.20$ & $<2.72$ & $<1.70$ & $<0.77$ & $<1.05$ \\
\hline 79 & 0 & 40 & 41.0 & 40 & 22 & 29 & 11 & 1 & 12.8 & $<1.26$ & $<0.49$ & $0.68 \pm 0.21$ & $<0.45$ & $0.44 \pm 0.16$ \\
\hline 80 & 0 & 40 & 41.8 & 41 & 8 & 8 & 19 & 1 & 10.8 & $<1.64$ & $<0.45$ & $<2.27$ & $<0.30$ & $<1.40$ \\
\hline 81 & 0 & 40 & 42.3 & 39 & 59 & 7 & 8 & 1 & 50.8 & $5.54 \pm 1.01$ & $<1.89$ & $3.97 \pm 0.77$ & $2.55 \pm 0.63$ & $1.54 \pm 0.47$ \\
\hline 82 & 0 & 40 & 42.3 & 40 & 32 & 41 & 12 & 1 & 10.4 & $<0.97$ & $<0.39$ & $<0.75$ & $<0.24$ & $0.54 \pm 0.16$ \\
\hline 83 & 0 & 40 & 44.3 & 39 & 37 & 1 & 12 & 4 & 135.1 & $11.01 \pm 1.57$ & $<0.85$ & $15.23 \pm 1.32$ & $6.17 \pm 0.88$ & $8.30 \pm 0.97$ \\
\hline 84 & 0 & 40 & 46.2 & 40 & 51 & 38 & 9 & 1 & 26.5 & $1.16 \pm 0.27$ & $<0.34$ & $0.98 \pm 0.21$ & $<0.44$ & $0.68 \pm 0.17$ \\
\hline 85 & 0 & 40 & 48.0 & 41 & 53 & 2 & 15 & 4 & 35.2 & $<0.81$ & $<0.11$ & $3.59 \pm 0.53$ & $1.21 \pm 0.31$ & $3.60 \pm 0.49$ \\
\hline 86 & 0 & 40 & 48.7 & 40 & 30 & 44 & 8 & 1 & 37.4 & $1.55 \pm 0.33$ & $<0.61$ & $1.00 \pm 0.22$ & $<0.28$ & $0.81 \pm 0.18$ \\
\hline 87 & 0 & 40 & 49.4 & 40 & 11 & 32 & 7 & 1 & 267.7 & $14.64 \pm 1.10$ & $9.49 \pm 0.90$ & $4.31 \pm 0.57$ & $1.98 \pm 0.39$ & $2.26 \pm 0.42$ \\
\hline 88 & 0 & 40 & 51.6 & 40 & 21 & 12 & 9 & 1 & 15.5 & $<1.04$ & $<0.23$ & $0.75 \pm 0.22$ & $<0.58$ & $<0.53$ \\
\hline 89 & 0 & 40 & 53.3 & 41 & 32 & 46 & 11 & 1 & 20.0 & $2.26 \pm 0.67$ & $<0.90$ & $2.03 \pm 0.54$ & $<1.20$ & $1.32 \pm 0.44$ \\
\hline 90 & 0 & 40 & 58.6 & 40 & 3 & 45 & 11 & 1 & 10.6 & $<2.23$ & $<0.67$ & $1.08 \pm 0.39$ & $<0.75$ & $<0.82$ \\
\hline 91 & 0 & 41 & 4.8 & 41 & 23 & 58 & 10 & 1 & 24.4 & $2.86 \pm 0.60$ & $<1.87$ & $2.12 \pm 0.48$ & $1.19 \pm 0.33$ & $<1.32$ \\
\hline 92 & 0 & 41 & 5.4 & 40 & 27 & 17 & 8 & 1 & 44.0 & $1.79 \pm 0.38$ & $<0.36$ & $1.43 \pm 0.28$ & $0.50 \pm 0.17$ & $0.90 \pm 0.22$ \\
\hline 93 & 0 & 41 & 6.9 & 40 & 2 & 51 & 10 & 1 & 45.4 & $5.57 \pm 1.01$ & $<1.49$ & $4.08 \pm 0.80$ & $1.42 \pm 0.47$ & $3.04 \pm 0.70$ \\
\hline 94 & 0 & 41 & 7.3 & 40 & 50 & 57 & 9 & 1 & 20.8 & $1.11 \pm 0.27$ & $<0.44$ & $0.72 \pm 0.18$ & $<0.33$ & $0.47 \pm 0.13$ \\
\hline 95 & 0 & 41 & 11.8 & 40 & 54 & 25 & 7 & 1 & 70.2 & $1.94 \pm 0.32$ & $<0.37$ & $1.60 \pm 0.25$ & $<0.45$ & $1.30 \pm 0.21$ \\
\hline 96 & 0 & 41 & 14.7 & 40 & 49 & 1 & 12 & 1 & 10.7 & $1.00 \pm 0.27$ & $<1.12$ & $<0.27$ & $<0.11$ & $<0.22$ \\
\hline 97 & 0 & 41 & 15.8 & 41 & 1 & 7 & 8 & 1 & 46.7 & $1.81 \pm 0.33$ & $<0.40$ & $1.37 \pm 0.24$ & $<0.65$ & $0.92 \pm 0.19$ \\
\hline 98 & 0 & 41 & 15.9 & 40 & 16 & 48 & 9 & 1 & 23.4 & $1.73 \pm 0.46$ & $<0.60$ & $1.26 \pm 0.32$ & $<0.78$ & $0.71 \pm 0.24$ \\
\hline 99 & 0 & 41 & 18.3 & 40 & 51 & 59 & 7 & 1 & 212.8 & $4.93 \pm 0.44$ & $<0.82$ & $3.88 \pm 0.36$ & $1.81 \pm 0.24$ & $2.14 \pm 0.27$ \\
\hline 100 & 0 & 41 & 24.8 & 41 & 36 & 51 & 11 & 1 & 12.4 & $<1.51$ & $<0.49$ & $1.07 \pm 0.34$ & $0.80 \pm 0.28$ & $<0.37$ \\
\hline 101 & 0 & 41 & 25.7 & 40 & 58 & 48 & 6 & 1 & 328.3 & $6.34 \pm 0.50$ & $<0.68$ & $5.04 \pm 0.41$ & $1.58 \pm 0.24$ & $3.52 \pm 0.34$ \\
\hline 102 & 0 & 41 & 26.0 & 40 & 53 & 30 & 7 & 1 & 87.6 & $2.78 \pm 0.36$ & $<0.70$ & $2.00 \pm 0.27$ & $0.97 \pm 0.19$ & $1.03 \pm 0.19$ \\
\hline 103 & 0 & 41 & 28.5 & 40 & 25 & 51 & 9 & 1 & 17.6 & $<1.37$ & $<0.36$ & $0.91 \pm 0.26$ & $<0.44$ & $0.58 \pm 0.20$ \\
\hline 104 & 0 & 41 & 31.3 & 41 & 5 & 55 & 6 & 1 & 901.2 & $12.36 \pm 0.69$ & $<0.19$ & $11.92 \pm 0.64$ & $2.84 \pm 0.32$ & $9.27 \pm 0.56$ \\
\hline 105 & 0 & 41 & 32.6 & 41 & 17 & 48 & 9 & 1 & 22.2 & $1.40 \pm 0.34$ & $<0.54$ & $0.99 \pm 0.24$ & $<0.41$ & $0.71 \pm 0.20$ \\
\hline 106 & 0 & 41 & 34.6 & 40 & 28 & 56 & 10 & 1 & 14.3 & $<1.50$ & $<0.70$ & $0.71 \pm 0.22$ & $<0.21$ & $0.57 \pm 0.19$ \\
\hline 107 & 0 & 41 & 35.9 & 41 & 40 & 6 & 11 & 1 & 14.3 & $<1.95$ & $<0.51$ & $1.03 \pm 0.35$ & $0.91 \pm 0.30$ & $<0.28$ \\
\hline 108 & 0 & 41 & 37.6 & 41 & 1 & 13 & 8 & 1 & 85.8 & $3.10 \pm 0.40$ & $<1.31$ & $2.30 \pm 0.30$ & $0.71 \pm 0.18$ & $1.56 \pm 0.24$ \\
\hline 109 & 0 & 41 & 41.1 & 40 & 31 & 22 & 10 & 1 & 28.4 & $1.75 \pm 0.42$ & $<0.54$ & $1.46 \pm 0.30$ & $0.64 \pm 0.20$ & $0.72 \pm 0.21$ \\
\hline 110 & 0 & 41 & 41.3 & 41 & 0 & 10 & 5 & 1 & 16.5 & $<2.12$ & $<0.33$ & $1.76 \pm 0.36$ & $<0.16$ & $0.56 \pm 0.16$ \\
\hline 111 & 0 & 41 & 41.4 & 41 & 3 & 32 & 8 & 1 & 46.6 & $1.84 \pm 0.33$ & $<0.36$ & $1.45 \pm 0.25$ & $0.66 \pm 0.17$ & $0.80 \pm 0.19$ \\
\hline 112 & 0 & 41 & 43.1 & 41 & 4 & 59 & 8 & 1 & 71.0 & $2.90 \pm 0.39$ & $<0.81$ & $2.06 \pm 0.29$ & $1.23 \pm 0.22$ & $0.82 \pm 0.19$ \\
\hline 113 & 0 & 41 & 43.2 & 41 & 34 & 22 & 6 & 1 & 3463.9 & $53.70 \pm 1.83$ & $<1.12$ & $51.53 \pm 1.75$ & $14.74 \pm 0.94$ & $37.07 \pm 1.49$ \\
\hline 114 & 0 & 41 & 44.1 & 41 & 21 & 19 & 10 & 1 & 26.2 & $1.11 \pm 0.33$ & $<0.62$ & $1.60 \pm 0.34$ & $<0.14$ & $0.92 \pm 0.23$ \\
\hline 115 & 0 & 41 & 44.7 & 40 & 22 & 0 & 5 & 1 & 52.1 & $19.66 \pm 1.52$ & $<3.94$ & $5.15 \pm 0.70$ & $2.37 \pm 0.48$ & $2.58 \pm 0.49$ \\
\hline 116 & 0 & 41 & 45.2 & 41 & 26 & 25 & 8 & 1 & 49.2 & $2.37 \pm 0.46$ & $<0.55$ & $1.91 \pm 0.35$ & $0.77 \pm 0.23$ & $1.01 \pm 0.25$ \\
\hline 117 & 0 & 41 & 45.5 & 40 & 34 & 31 & 9 & 1 & 20.6 & $<1.27$ & $<0.30$ & $0.88 \pm 0.22$ & $<0.64$ & $0.42 \pm 0.15$ \\
\hline 118 & 0 & 41 & 45.7 & 40 & 36 & 24 & 10 & 1 & 10.6 & $<0.98$ & $<0.92$ & $<0.56$ & $<0.12$ & $0.33 \pm 0.13$ \\
\hline 119 & 0 & 41 & 47.2 & 41 & 31 & 55 & 9 & 1 & 53.5 & $3.08 \pm 0.56$ & $<0.54$ & $3.00 \pm 0.46$ & $0.95 \pm 0.27$ & $1.62 \pm 0.34$ \\
\hline 120 & 0 & 41 & 48.7 & 40 & 28 & 14 & 10 & 1 & 13.0 & $<1.08$ & $<0.42$ & $0.73 \pm 0.25$ & $<0.27$ & $0.61 \pm 0.21$ \\
\hline 121 & 0 & 41 & 48.9 & 41 & 22 & 33 & 7 & 1 & 94.1 & $2.69 \pm 0.43$ & $<0.30$ & $2.57 \pm 0.37$ & $<0.70$ & $2.03 \pm 0.32$ \\
\hline 122 & 0 & 41 & 49.6 & 41 & 1 & 8 & 6 & 1 & 299.0 & $6.25 \pm 0.51$ & $<0.58$ & $5.22 \pm 0.43$ & $1.69 \pm 0.24$ & $3.62 \pm 0.35$ \\
\hline 123 & 0 & 41 & 49.9 & 40 & 15 & 26 & 8 & 1 & 62.0 & $7.02 \pm 1.04$ & $2.80 \pm 0.76$ & $3.60 \pm 0.68$ & $<2.33$ & $2.20 \pm 0.51$ \\
\hline 124 & 0 & 41 & 50.6 & 41 & 13 & 34 & 9 & 1 & 18.3 & $<1.25$ & $<0.20$ & $0.83 \pm 0.22$ & $<0.26$ & $0.71 \pm 0.19$ \\
\hline 125 & 0 & 41 & 51.3 & 41 & 51 & 53 & 11 & 1 & 14.3 & $3.28 \pm 0.91$ & $<1.80$ & $1.94 \pm 0.66$ & $<1.43$ & $<1.17$ \\
\hline 126 & 0 & 41 & 51.9 & 41 & 14 & 49 & 8 & 1 & 46.2 & $1.85 \pm 0.36$ & $<0.40$ & $1.84 \pm 0.31$ & $0.63 \pm 0.18$ & $1.21 \pm 0.25$ \\
\hline
\end{tabular}


Table 5. continued.

$\star$ Bulge sources

\begin{tabular}{|c|c|c|c|c|c|c|c|c|c|c|c|c|c|c|}
\hline $\begin{array}{l}S I I \\
\text { No. } \\
(1)\end{array}$ & $\begin{array}{l}\text { I } \\
\text { (h) } \\
\text { (2) }\end{array}$ & $\begin{array}{l}\text { A } \\
(\mathrm{m}) \\
(3)\end{array}$ & $\begin{array}{l}(\mathrm{J} 200 \\
(\mathrm{s}) \\
(4)\end{array}$ & $\begin{array}{l}00) \\
\left(\begin{array}{l}0 \\
(5) \\
(5)\end{array}\right.\end{array}$ & $\begin{array}{l}\text { De } \\
\left({ }^{\prime}\right) \\
(6)\end{array}$ & $\begin{array}{l}\text { ec } \\
\left({ }^{\prime \prime}\right) \\
(7)\end{array}$ & $\begin{array}{r}\sigma_{\mathrm{Pos}} \\
\left({ }^{\prime \prime}\right) \\
(8)\end{array}$ & $\begin{array}{l}\text { Cl. } \\
(9)\end{array}$ & $\begin{array}{c}\text { Maxlik } \\
(\mathrm{LH}) \\
(10)\end{array}$ & $\begin{array}{l}\text { Rate }(B) \\
\left(\mathrm{ct} \cdot \mathrm{ks}^{-1}\right) \\
\quad(11)\end{array}$ & $\begin{array}{c}\text { Rate }(S) \\
\left(\mathrm{ct} \cdot \mathrm{ks}^{-1}\right) \\
(12)\end{array}$ & $\begin{array}{c}\text { Rate }(H) \\
\left(\mathrm{ct} \cdot \mathrm{ks}^{-1}\right) \\
(13)\end{array}$ & $\begin{array}{c}\text { Rate }\left(H_{1}\right) \\
\left(\mathrm{ct} \cdot \mathrm{ks}^{-1}\right) \\
(14)\end{array}$ & $\begin{array}{c}\text { Rate }\left(H_{2}\right) \\
\left(\mathrm{ct} \cdot \mathrm{ks}^{-1}\right) \\
(15)\end{array}$ \\
\hline 127 & 0 & 41 & 52.6 & 40 & 21 & 24 & 6 & 1 & 3614.5 & $90.56 \pm 2.85$ & $10.27 \pm 1.06$ & $76.44 \pm 2.59$ & $36.93 \pm 1.80$ & $41.22 \pm 1.91$ \\
\hline 128 & 0 & 41 & 54.7 & 41 & 33 & 38 & 8 & 1 & 45.1 & $2.10 \pm 0.44$ & $<0.48$ & $1.76 \pm 0.34$ & $0.78 \pm 0.23$ & $0.91 \pm 0.24$ \\
\hline 129 & 0 & 41 & 55.6 & 40 & 46 & 49 & 15 & 1 & 15.1 & $<2.96$ & $<0.17$ & $2.82 \pm 0.42$ & $<0.92$ & $1.22 \pm 0.25$ \\
\hline 130 & 0 & 42 & 0.7 & 40 & 41 & 24 & 7 & 1 & 142.7 & $6.47 \pm 0.61$ & $5.62 \pm 0.58$ & $1.44 \pm 0.27$ & $1.09 \pm 0.23$ & $<0.49$ \\
\hline 131 & 0 & 42 & 2.6 & 40 & 31 & 18 & 10 & 1 & 16.0 & $<1.40$ & $<0.49$ & $0.77 \pm 0.24$ & $<0.52$ & $<0.57$ \\
\hline 132 & 0 & 42 & 2.7 & 40 & 46 & 15 & 8 & 1 & 42.1 & $1.50 \pm 0.32$ & $<0.44$ & $1.06 \pm 0.22$ & $<0.83$ & $0.91 \pm 0.19$ \\
\hline 133 & 0 & 42 & 3.0 & 40 & 24 & 16 & 10 & 1 & 45.9 & $3.24 \pm 0.68$ & $<0.62$ & $3.79 \pm 0.64$ & $1.56 \pm 0.41$ & $2.06 \pm 0.47$ \\
\hline 134 & 0 & 42 & 3.2 & 40 & 28 & 56 & 8 & 1 & 46.0 & $2.19 \pm 0.47$ & $<0.37$ & $1.84 \pm 0.37$ & $0.77 \pm 0.24$ & $1.03 \pm 0.28$ \\
\hline 135 & 0 & 42 & 3.3 & 40 & 33 & 8 & 10 & 1 & 12.3 & $<1.15$ & $<0.39$ & $0.67 \pm 0.22$ & $<0.66$ & $<0.32$ \\
\hline 136 & 0 & 42 & 6.3 & 41 & 2 & 47 & 8 & 1 & 37.4 & $2.17 \pm 0.37$ & $<1.76$ & $1.39 \pm 0.25$ & $<0.69$ & $0.93 \pm 0.20$ \\
\hline 137 & 0 & 42 & 7.1 & 41 & 0 & 19 & 8 & 1 & 66.3 & $2.37 \pm 0.37$ & $<0.43$ & $2.16 \pm 0.31$ & $<0.46$ & $2.01 \pm 0.28$ \\
\hline 138 & 0 & 42 & 7.8 & 41 & 4 & 39 & 8 & 1 & 63.1 & $3.20 \pm 0.43$ & $<2.79$ & $2.13 \pm 0.31$ & $0.88 \pm 0.20$ & $1.19 \pm 0.23$ \\
\hline 139 & 0 & 42 & 9.0 & 41 & 18 & 19 & 5 & 1 & 970.6 & $35.01 \pm 1.21$ & $<0.32$ & $27.80 \pm 1.01$ & $5.76 \pm 0.48$ & $21.32 \pm 0.88$ \\
\hline 140 & 0 & 42 & 9.3 & 41 & 20 & 58 & 10 & 1 & 13.5 & $1.33 \pm 0.36$ & $<0.50$ & $0.99 \pm 0.26$ & $<0.48$ & $<0.84$ \\
\hline 141 & 0 & 42 & 9.6 & 40 & 16 & 47 & 9 & 1 & 36.0 & $4.96 \pm 1.07$ & $<1.17$ & $3.77 \pm 0.83$ & $1.66 \pm 0.55$ & $2.06 \pm 0.60$ \\
\hline 142 & 0 & 42 & 10.9 & 41 & 10 & 46 & 9 & 1 & 80.0 & $6.83 \pm 0.65$ & $<1.58$ & $3.65 \pm 0.42$ & $1.32 \pm 0.27$ & $2.33 \pm 0.33$ \\
\hline 143 & 0 & 42 & 12.0 & 41 & 15 & 17 & 5 & 1 & 34.7 & $<3.37$ & $<0.41$ & $4.53 \pm 0.50$ & $<1.60$ & $2.82 \pm 0.38$ \\
\hline 144 & 0 & 42 & 12.4 & 41 & 18 & 31 & 5 & 1 & 1492.6 & $36.25 \pm 1.21$ & $<0.38$ & $33.07 \pm 1.10$ & $8.65 \pm 0.60$ & $29.48 \pm 1.04$ \\
\hline 145 & 0 & 42 & 13.5 & 40 & 39 & 25 & 6 & 1 & 329.3 & $8.12 \pm 0.71$ & $<1.30$ & $6.62 \pm 0.59$ & $2.20 \pm 0.34$ & $4.45 \pm 0.48$ \\
\hline 146 & 0 & 42 & 15.2 & 40 & 19 & 45 & 6 & 1 & 3894.8 & $150.77 \pm 4.58$ & $14.56 \pm 1.57$ & $125.26 \pm 4.09$ & $43.66 \pm 2.43$ & $80.55 \pm 3.27$ \\
\hline 147 & 0 & 42 & 15.6 & 41 & 1 & 16 & 6 & 1 & 2651.4 & $29.59 \pm 1.02$ & $<0.83$ & $27.56 \pm 0.95$ & $3.63 \pm 0.36$ & $23.98 \pm 0.88$ \\
\hline 148 & 0 & 42 & 15.6 & 41 & 20 & 31 & 8 & 1 & 47.9 & $2.06 \pm 0.37$ & $<0.27$ & $1.79 \pm 0.30$ & $0.56 \pm 0.17$ & $1.18 \pm 0.24$ \\
\hline 149 & 0 & 42 & 16.0 & 41 & 31 & 19 & 11 & 1 & 11.1 & $<0.96$ & $<0.26$ & $0.81 \pm 0.24$ & $<0.52$ & $<0.59$ \\
\hline 150 & 0 & 42 & 16.2 & 40 & 55 & 54 & 6 & 1 & 362.7 & $6.59 \pm 0.52$ & $<0.44$ & $5.83 \pm 0.45$ & $1.53 \pm 0.24$ & $4.38 \pm 0.39$ \\
\hline 151 & 0 & 42 & 16.3 & 40 & 48 & 18 & 11 & 1 & 13.9 & $<1.22$ & $<0.43$ & $0.84 \pm 0.22$ & $0.57 \pm 0.17$ & $<0.27$ \\
\hline 152 & 0 & 42 & 16.6 & 41 & 29 & 1 & 12 & 1 & 13.8 & $<0.83$ & $<0.65$ & $<1.45$ & $<0.21$ & $1.17 \pm 0.27$ \\
\hline 153 & 0 & 42 & 17.7 & 41 & 12 & 29 & 5 & 1 & 2282.0 & $33.74 \pm 0.95$ & $3.22 \pm 0.51$ & $35.36 \pm 1.11$ & $13.32 \pm 0.68$ & $21.68 \pm 0.87$ \\
\hline 154 & 0 & 42 & 19.3 & 41 & 14 & 0 & 5 & 1 & 812.8 & $66.18 \pm 0.96$ & $2.59 \pm 0.50$ & $22.41 \pm 0.80$ & $9.39 \pm 0.60$ & $18.04 \pm 0.82$ \\
\hline 155 & 0 & 42 & 20.2 & 41 & 47 & 46 & 12 & 1 & 10.1 & $<1.12$ & $<0.39$ & $0.79 \pm 0.30$ & $<0.47$ & $<0.72$ \\
\hline 156 & 0 & 42 & 20.3 & 41 & 26 & 41 & 9 & 1 & 27.9 & $1.69 \pm 0.37$ & $<0.40$ & $1.37 \pm 0.28$ & $<0.63$ & $0.91 \pm 0.23$ \\
\hline 157 & 0 & 42 & 22.2 & 40 & 59 & 25 & 6 & 1 & 589.7 & $9.73 \pm 0.62$ & $<0.57$ & $8.72 \pm 0.55$ & $2.89 \pm 0.32$ & $5.86 \pm 0.45$ \\
\hline 158 & 0 & 42 & 22.6 & 40 & 44 & 18 & 9 & 1 & 35.1 & $1.26 \pm 0.32$ & $<0.48$ & $0.96 \pm 0.23$ & $<0.19$ & $0.90 \pm 0.21$ \\
\hline$\star 159$ & 0 & 42 & 22.8 & 41 & 15 & 43 & 5 & 1 & 2830.2 & $71.08 \pm 0.81$ & $1.68 \pm 0.38$ & $44.28 \pm 1.06$ & $16.12 \pm 0.74$ & $33.13 \pm 1.05$ \\
\hline$\star 160$ & 0 & 42 & 22.9 & 40 & 19 & 42 & 7 & 1 & 815.9 & $94.42 \pm 4.08$ & $6.63 \pm 1.45$ & $48.60 \pm 2.78$ & $16.05 \pm 1.63$ & $30.90 \pm 2.20$ \\
\hline 161 & 0 & 42 & 22.9 & 41 & 13 & 34 & 5 & 1 & 651.3 & $93.27 \pm 1.12$ & $3.55 \pm 0.53$ & $75.40 \pm 1.45$ & $59.85 \pm 1.46$ & $52.47 \pm 1.39$ \\
\hline 162 & 0 & 42 & 23.1 & 41 & 7 & 33 & 9 & 1 & 21.1 & $1.65 \pm 0.35$ & $<0.71$ & $0.92 \pm 0.23$ & $<1.29$ & $0.59 \pm 0.17$ \\
\hline 163 & 0 & 42 & 24.3 & 40 & 57 & 17 & 9 & 1 & 20.2 & $<1.22$ & $<0.23$ & $0.84 \pm 0.20$ & $<0.40$ & $0.58 \pm 0.16$ \\
\hline 164 & 0 & 42 & 24.8 & 39 & 47 & 38 & 26 & 4 & 10.2 & $<0.78$ & $<0.32$ & $<3.91$ & $<0.85$ & $2.58 \pm 0.67$ \\
\hline 165 & 0 & 42 & 25.0 & 40 & 28 & 30 & 14 & 1 & 12.3 & $<2.00$ & $<0.35$ & $<2.31$ & $<0.33$ & $<2.18$ \\
\hline 166 & 0 & 42 & 26.4 & 41 & 25 & 54 & 7 & 1 & 128.8 & $4.21 \pm 0.50$ & $<0.25$ & $3.95 \pm 0.43$ & $0.85 \pm 0.21$ & $3.02 \pm 0.37$ \\
\hline 167 & 0 & 42 & 26.9 & 41 & 45 & 30 & 10 & 1 & 15.3 & $<1.04$ & $<0.22$ & $1.06 \pm 0.31$ & $<0.56$ & $0.66 \pm 0.23$ \\
\hline$\star 168$ & 0 & 42 & 27.6 & 41 & 19 & 21 & 5 & 1 & 131.9 & $9.20 \pm 0.62$ & $<0.40$ & $8.01 \pm 0.59$ & $2.25 \pm 0.34$ & $5.37 \pm 0.48$ \\
\hline 169 & 0 & 42 & 28.4 & 41 & 12 & 22 & 5 & 1 & 1533.1 & $44.15 \pm 0.76$ & $4.08 \pm 0.54$ & $26.47 \pm 0.92$ & $9.74 \pm 0.59$ & $16.72 \pm 0.76$ \\
\hline 170 & 0 & 42 & 29.1 & 41 & 4 & 35 & 6 & 1 & 1118.4 & $17.85 \pm 0.83$ & $<1.17$ & $15.22 \pm 0.74$ & $5.04 \pm 0.43$ & $10.24 \pm 0.60$ \\
\hline 171 & 0 & 42 & 29.2 & 41 & 8 & 26 & 8 & 1 & 43.0 & $2.41 \pm 0.39$ & $<2.06$ & $1.59 \pm 0.28$ & $0.64 \pm 0.18$ & $0.98 \pm 0.21$ \\
\hline 172 & 0 & 42 & 29.6 & 41 & 29 & 3 & 8 & 1 & 51.7 & $1.52 \pm 0.35$ & $<0.39$ & $1.63 \pm 0.28$ & $0.54 \pm 0.17$ & $1.15 \pm 0.23$ \\
\hline$\star 173$ & 0 & 42 & 29.7 & 41 & 14 & 30 & 5 & 1 & 317.8 & $154.67 \pm 1.01$ & $7.28 \pm 0.68$ & $97.14 \pm 1.08$ & $35.21 \pm 1.10$ & $42.28 \pm 1.04$ \\
\hline$\star 174$ & 0 & 42 & 31.4 & 41 & 16 & 17 & 5 & 1 & 897.3 & $51.43 \pm 0.57$ & $8.92 \pm 0.71$ & $104.70 \pm 0.95$ & $37.20 \pm 1.02$ & $20.30 \pm 0.67$ \\
\hline$\star 175$ & 0 & 42 & 32.1 & 41 & 19 & 35 & 5 & 1 & 263.9 & $8.48 \pm 0.56$ & $<0.51$ & $10.64 \pm 0.66$ & $3.54 \pm 0.39$ & $7.42 \pm 0.56$ \\
\hline 176 & 0 & 42 & 32.2 & 41 & 55 & 45 & 9 & 1 & 38.1 & $3.64 \pm 0.77$ & $<1.11$ & $2.61 \pm 0.56$ & $1.61 \pm 0.44$ & $0.88 \pm 0.34$ \\
\hline$\star 177$ & 0 & 42 & 32.3 & 41 & 13 & 16 & 5 & 1 & 611.1 & $121.82 \pm 0.99$ & $7.95 \pm 0.67$ & $53.14 \pm 1.22$ & $23.41 \pm 0.94$ & $35.42 \pm 1.14$ \\
\hline 178 & 0 & 42 & 33.5 & 41 & 21 & 49 & 10 & 1 & 18.0 & $1.30 \pm 0.34$ & $<0.24$ & $1.16 \pm 0.26$ & $0.85 \pm 0.21$ & $<0.41$ \\
\hline 179 & 0 & 42 & 34.4 & 41 & 32 & 53 & 6 & 1 & 287.1 & $6.30 \pm 0.58$ & $<0.45$ & $5.68 \pm 0.51$ & $0.91 \pm 0.21$ & $4.93 \pm 0.47$ \\
\hline 180 & 0 & 42 & 34.5 & 40 & 48 & 40 & 7 & 1 & 171.8 & $3.91 \pm 0.46$ & $<0.33$ & $3.54 \pm 0.39$ & $1.34 \pm 0.24$ & $2.28 \pm 0.31$ \\
\hline 181 & 0 & 42 & 35.0 & 40 & 40 & 35 & 11 & 1 & 12.9 & $<1.26$ & $<0.44$ & $0.82 \pm 0.25$ & $<0.41$ & $0.60 \pm 0.21$ \\
\hline$\star 182$ & 0 & 42 & 36.7 & 41 & 14 & 1 & 5 & 1 & 611.8 & $171.71 \pm 0.99$ & $10.17 \pm 0.76$ & $93.39 \pm 1.05$ & $29.82 \pm 0.93$ & $33.16 \pm 0.92$ \\
\hline 183 & 0 & 42 & 38.8 & 41 & 10 & 16 & 11 & 1 & 18.4 & $6.38 \pm 0.66$ & $<2.67$ & $2.07 \pm 0.40$ & $1.59 \pm 0.32$ & $<0.61$ \\
\hline$\star 184$ & 0 & 42 & 39.1 & 41 & 16 & 3 & 5 & 1 & 4245.6 & $361.41 \pm 1.29$ & $26.98 \pm 1.03$ & $242.68 \pm 1.24$ & $74.53 \pm 1.25$ & $138.36 \pm 1.21$ \\
\hline 185 & 0 & 42 & 39.4 & 41 & 59 & 52 & 13 & 1 & 19.2 & $3.04 \pm 0.84$ & $<1.04$ & $3.74 \pm 0.81$ & $1.38 \pm 0.48$ & $<1.14$ \\
\hline 186 & 0 & 42 & 39.5 & 40 & 43 & 20 & 8 & 1 & 75.2 & $3.96 \pm 0.53$ & $1.36 \pm 0.36$ & $2.43 \pm 0.37$ & $1.44 \pm 0.28$ & $0.97 \pm 0.24$ \\
\hline$\star 187$ & 0 & 42 & 40.4 & 41 & 13 & 29 & 5 & 1 & 701.9 & $112.89 \pm 0.87$ & $7.23 \pm 0.65$ & $61.78 \pm 0.87$ & $26.80 \pm 1.00$ & $32.18 \pm 1.09$ \\
\hline 188 & 0 & 42 & 41.8 & 40 & 51 & 53 & 5 & 1 & 5446.2 & $58.12 \pm 1.50$ & $4.21 \pm 0.47$ & $51.83 \pm 1.38$ & $18.10 \pm 0.81$ & $33.92 \pm 1.12$ \\
\hline 189 & 0 & 42 & 41.8 & 42 & 3 & 16 & 13 & 1 & 13.1 & $<3.45$ & $<1.00$ & $2.01 \pm 0.66$ & $<1.23$ & $<1.79$ \\
\hline$\star 190$ & 0 & 42 & 41.9 & 41 & 18 & 26 & 5 & 1 & 215.8 & $81.41 \pm 0.78$ & $4.20 \pm 0.5$ & $47.10 \pm 0.80$ & $19.54 \pm 0.86$ & $22.07 \pm 0.93$ \\
\hline 191 & 0 & 42 & 42.7 & 41 & 28 & 21 & 12 & 1 & 17.6 & $<0.56$ & $<0.31$ & $<1.29$ & $<0.19$ & $1.23 \pm 0.26$ \\
\hline 192 & 0 & 42 & 43.5 & 40 & 8 & 34 & 24 & 4 & 10.4 & $<0.84$ & $<0.12$ & $<1.82$ & $<1.18$ & $1.33 \pm 0.33$ \\
\hline 193 & 0 & 42 & 44.1 & 41 & 21 & 28 & 11 & 1 & 31.1 & $5.61 \pm 0.64$ & $<0.59$ & $3.46 \pm 0.46$ & $<2.41$ & $1.55 \pm 0.30$ \\
\hline$\star 194$ & 0 & 42 & 44.6 & 41 & 11 & 43 & 6 & 1 & 1077.8 & $17.36 \pm 0.69$ & $3.62 \pm 0.53$ & $18.33 \pm 0.80$ & $8.14 \pm 0.54$ & $9.74 \pm 0.58$ \\
\hline
\end{tabular}


Table 5. continued.

\begin{tabular}{|c|c|c|c|c|c|c|c|c|c|c|c|c|c|c|}
\hline $\begin{array}{l}S I I \\
\text { No. } \\
(1)\end{array}$ & $\begin{array}{l}{ }^{\mathrm{I}} \\
(\mathrm{h}) \\
(2)\end{array}$ & $\begin{array}{l}\text { A } \\
(\mathrm{m}) \\
(3)\end{array}$ & $\begin{array}{l}(\mathrm{J} 20 \\
(\mathrm{s}) \\
(4)\end{array}$ & $\begin{array}{l}\text { 0) } \\
\left({ }^{\circ}\right) \\
(5)\end{array}$ & $\begin{array}{r}\left({ }^{\prime}\right) \\
(6)\end{array}$ & $\begin{array}{l}\mathrm{c} \\
\left({ }^{\prime \prime}\right) \\
(7)\end{array}$ & $\begin{array}{r}\sigma_{\text {Pos }} \\
\left({ }^{\prime \prime}\right) \\
(8)\end{array}$ & $\begin{array}{l}\mathrm{Cl} . \\
(9)\end{array}$ & $\begin{array}{c}\text { Maxlik } \\
(\mathrm{LH}) \\
(10)\end{array}$ & $\begin{array}{c}\text { Rate }(B) \\
\left(\mathrm{ct} \cdot \mathrm{ks}^{-1}\right) \\
(11)\end{array}$ & $\begin{array}{c}\text { Rate }(S) \\
\left(\mathrm{ct} \cdot \mathrm{ks}^{-1}\right) \\
(12)\end{array}$ & $\begin{array}{c}\text { Rate }(H) \\
\left(\mathrm{ct} \cdot \mathrm{ks}^{-1}\right) \\
(13)\end{array}$ & $\begin{array}{c}\text { Rate }\left(H_{1}\right) \\
\left(\mathrm{ct} \cdot \mathrm{ks}^{-1}\right) \\
(14)\end{array}$ & $\begin{array}{c}\operatorname{Rate}\left(H_{2}\right) \\
\left(\mathrm{ct} \cdot \mathrm{ks}^{-1}\right) \\
(15)\end{array}$ \\
\hline$\star 195$ & 0 & 42 & 45.5 & 41 & 16 & 28 & 5 & 1 & 2468.3 & $332.40 \pm 1.24$ & $39.91 \pm 1.24$ & $278.71 \pm 1.33$ & $55.02 \pm 1.07$ & $141.34 \pm 1.23$ \\
\hline 196 & 0 & 42 & 47.2 & 40 & 58 & 32 & 10 & 1 & 12.2 & $<1.38$ & $<0.65$ & $0.60 \pm 0.18$ & $<0.47$ & $<0.40$ \\
\hline$\star 197$ & 0 & 42 & 47.3 & 41 & 15 & 24 & 5 & 1 & 1843.7 & $423.34 \pm 1.43$ & $48.24 \pm 1.36$ & $198.84 \pm 1.13$ & $68.29 \pm 1.21$ & $94.76 \pm 1.02$ \\
\hline 198 & 0 & 42 & 48.6 & 41 & 25 & 26 & 6 & 1 & 776.3 & $21.27 \pm 0.98$ & $<0.60$ & $15.13 \pm 0.76$ & $3.68 \pm 0.40$ & $11.12 \pm 0.64$ \\
\hline 199 & 0 & 42 & 51.6 & 41 & 31 & 9 & 5 & 1 & 6523.9 & $66.34 \pm 1.60$ & $<0.76$ & $63.28 \pm 1.52$ & $14.96 \pm 0.74$ & $48.51 \pm 1.34$ \\
\hline$\star 200$ & 0 & 42 & 52.2 & 41 & 18 & 57 & 5 & 1 & 1868.8 & $71.46 \pm 0.82$ & $3.50 \pm 0.53$ & $59.15 \pm 1.37$ & $26.19 \pm 1.00$ & $22.63 \pm 0.88$ \\
\hline 201 & 0 & 42 & 52.7 & 40 & 53 & 27 & 10 & 1 & 11.6 & $<0.92$ & $<0.29$ & $0.58 \pm 0.18$ & $<0.35$ & $<0.51$ \\
\hline 202 & 0 & 42 & 53.2 & 40 & 13 & 20 & 11 & 4 & 135.7 & $<1.81$ & $<0.09$ & $7.40 \pm 0.58$ & $4.00 \pm 0.42$ & $5.53 \pm 0.47$ \\
\hline$\star 203$ & 0 & 42 & 53.3 & 41 & 15 & 49 & 5 & 1 & 1250.4 & $253.68 \pm 1.13$ & $30.40 \pm 1.09$ & $136.85 \pm 1.07$ & $45.88 \pm 1.01$ & $45.55 \pm 1.03$ \\
\hline 204 & 0 & 42 & 53.6 & 40 & 48 & 18 & 10 & 1 & 10.4 & $<0.95$ & $<0.35$ & $<0.73$ & $<0.39$ & $0.36 \pm 0.14$ \\
\hline 205 & 0 & 42 & 53.7 & 41 & 49 & 32 & 5 & 1 & 13.2 & $<2.38$ & $<0.92$ & $1.59 \pm 0.40$ & $<1.31$ & $<0.65$ \\
\hline 206 & 0 & 42 & 54.4 & 41 & 25 & 55 & 6 & 1 & 606.8 & $11.39 \pm 0.72$ & $<0.36$ & $12.22 \pm 0.69$ & $3.96 \pm 0.40$ & $8.20 \pm 0.56$ \\
\hline$\star 207$ & 0 & 42 & 54.5 & 41 & 13 & 36 & 5 & 1 & 98.0 & $65.09 \pm 0.82$ & $4.70 \pm 0.57$ & $8.44 \pm 0.53$ & $6.73 \pm 0.52$ & $1.46 \pm 0.27$ \\
\hline$\star 208$ & 0 & 42 & 56.1 & 41 & 17 & 1 & 5 & 1 & 298.8 & $31.59 \pm 0.48$ & $15.27 \pm 0.86$ & $92.18 \pm 1.06$ & $35.71 \pm 1.03$ & $40.83 \pm 1.09$ \\
\hline 209 & 0 & 42 & 57.4 & 41 & 11 & 5 & 6 & 1 & 857.1 & $16.97 \pm 0.81$ & $1.10 \pm 0.31$ & $13.15 \pm 0.67$ & $4.96 \pm 0.42$ & $8.10 \pm 0.53$ \\
\hline 210 & 0 & 42 & 57.8 & 41 & 46 & 6 & 7 & 1 & 212.7 & $7.64 \pm 0.72$ & $2.11 \pm 0.47$ & $5.17 \pm 0.55$ & $3.04 \pm 0.42$ & $2.16 \pm 0.36$ \\
\hline$\star 211$ & 0 & 42 & 58.8 & 41 & 19 & 11 & 5 & 1 & 618.2 & $79.32 \pm 1.06$ & $4.33 \pm 0.59$ & $20.47 \pm 0.86$ & $17.73 \pm 0.87$ & $10.68 \pm 0.64$ \\
\hline 212 & 0 & 43 & 1.0 & 41 & 30 & 17 & 7 & 1 & 249.9 & $6.87 \pm 0.58$ & $<0.44$ & $6.06 \pm 0.50$ & $1.50 \pm 0.26$ & $4.33 \pm 0.42$ \\
\hline$\star 213$ & 0 & 43 & 1.5 & 41 & 13 & 55 & 5 & 1 & 138.3 & $57.23 \pm 0.95$ & $<2.66$ & $9.58 \pm 0.58$ & $4.96 \pm 0.45$ & $4.49 \pm 0.44$ \\
\hline$\star 214$ & 0 & 43 & 1.6 & 41 & 15 & 32 & 5 & 1 & 1555.2 & $133.44 \pm 1.01$ & $8.41 \pm 0.69$ & $35.88 \pm 0.89$ & $32.31 \pm 1.08$ & $27.70 \pm 0.96$ \\
\hline 215 & 0 & 43 & 1.9 & 40 & 44 & 58 & 9 & 1 & 57.5 & $2.99 \pm 0.54$ & $<0.48$ & $2.83 \pm 0.43$ & $0.94 \pm 0.25$ & $1.74 \pm 0.33$ \\
\hline 216 & 0 & 43 & 2.9 & 41 & 21 & 20 & 7 & 1 & 232.3 & $9.74 \pm 0.61$ & $<1.16$ & $6.94 \pm 0.54$ & $3.36 \pm 0.37$ & $3.26 \pm 0.37$ \\
\hline$\star 217$ & 0 & 43 & 3.0 & 41 & 18 & 4 & 5 & 1 & 843.9 & $77.56 \pm 0.90$ & $7.31 \pm 0.65$ & $36.56 \pm 1.08$ & $14.76 \pm 0.78$ & $24.42 \pm 0.99$ \\
\hline 218 & 0 & 43 & 7.7 & 41 & 45 & 51 & 8 & 1 & 28.9 & $1.55 \pm 0.38$ & $<0.57$ & $1.12 \pm 0.26$ & $<0.38$ & $0.85 \pm 0.22$ \\
\hline 219 & 0 & 43 & 7.8 & 41 & 12 & 58 & 5 & 1 & 60.8 & $5.63 \pm 0.56$ & $<0.52$ & $2.17 \pm 0.31$ & $1.70 \pm 0.28$ & $0.81 \pm 0.20$ \\
\hline 220 & 0 & 43 & 8.8 & 41 & 52 & 8 & 9 & 1 & 22.5 & $1.40 \pm 0.42$ & $<0.43$ & $1.10 \pm 0.29$ & $<0.62$ & $0.92 \pm 0.28$ \\
\hline 221 & 0 & 43 & 9.3 & 40 & 59 & 28 & 8 & 1 & 27.5 & $1.50 \pm 0.33$ & $<1.04$ & $0.90 \pm 0.21$ & $<0.32$ & $0.68 \pm 0.17$ \\
\hline 222 & 0 & 43 & 9.5 & 41 & 19 & 0 & 5 & 1 & 1217.7 & $28.17 \pm 0.91$ & $2.91 \pm 0.54$ & $24.21 \pm 0.94$ & $12.81 \pm 0.74$ & $15.33 \pm 0.75$ \\
\hline 223 & 0 & 43 & 10.4 & 41 & 14 & 49 & 5 & 1 & 2311.9 & $31.25 \pm 0.89$ & $2.87 \pm 0.44$ & $32.60 \pm 1.05$ & $12.33 \pm 0.65$ & $19.83 \pm 0.82$ \\
\hline 224 & 0 & 43 & 10.7 & 41 & 55 & 41 & 10 & 1 & 18.4 & $<1.68$ & $<0.47$ & $1.03 \pm 0.30$ & $<0.33$ & $0.82 \pm 0.26$ \\
\hline 225 & 0 & 43 & 11.8 & 40 & 48 & 35 & 7 & 1 & 169.0 & $5.53 \pm 0.62$ & $<1.03$ & $4.31 \pm 0.50$ & $1.70 \pm 0.31$ & $2.62 \pm 0.39$ \\
\hline 226 & 0 & 43 & 13.4 & 41 & 17 & 15 & 5 & 1 & 73.1 & $11.08 \pm 0.69$ & $3.55 \pm 0.53$ & $6.00 \pm 0.55$ & $3.04 \pm 0.39$ & $2.60 \pm 0.37$ \\
\hline 227 & 0 & 43 & 13.9 & 41 & 7 & 21 & 6 & 1 & 634.7 & $11.88 \pm 0.70$ & $<1.01$ & $10.31 \pm 0.62$ & $2.91 \pm 0.34$ & $7.44 \pm 0.53$ \\
\hline 228 & 0 & 43 & 15.4 & 40 & 54 & 6 & 11 & 1 & 10.8 & $<1.02$ & $<0.42$ & $0.53 \pm 0.18$ & $<0.34$ & $<0.44$ \\
\hline 229 & 0 & 43 & 15.5 & 41 & 3 & 43 & 10 & 1 & 17.0 & $1.24 \pm 0.32$ & $<0.73$ & $0.74 \pm 0.20$ & $<0.40$ & $0.49 \pm 0.16$ \\
\hline 230 & 0 & 43 & 15.6 & 41 & 23 & 41 & 9 & 1 & 75.6 & $6.34 \pm 0.63$ & $4.29 \pm 0.49$ & $<2.94$ & $<1.97$ & $<0.64$ \\
\hline 231 & 0 & 43 & 17.2 & 41 & 12 & 28 & 12 & 1 & 12.7 & $1.53 \pm 0.38$ & $<0.34$ & $<1.43$ & $0.87 \pm 0.22$ & $<0.28$ \\
\hline 232 & 0 & 43 & 17.2 & 41 & 27 & 44 & 6 & 1 & 1165.6 & $17.30 \pm 0.81$ & $<0.88$ & $15.37 \pm 0.73$ & $5.61 \pm 0.44$ & $9.78 \pm 0.59$ \\
\hline 233 & 0 & 43 & 18.0 & 41 & 59 & 8 & 7 & 1 & 185.4 & $7.12 \pm 0.84$ & $<0.45$ & $6.65 \pm 0.74$ & $2.12 \pm 0.43$ & $4.53 \pm 0.61$ \\
\hline 234 & 0 & 43 & 19.4 & 40 & 48 & 38 & 10 & 1 & 21.6 & $<1.85$ & $<0.52$ & $1.39 \pm 0.33$ & $<0.53$ & $0.90 \pm 0.25$ \\
\hline 235 & 0 & 43 & 20.2 & 41 & 20 & 25 & 10 & 1 & 42.6 & $10.07 \pm 0.91$ & $<1.26$ & $4.29 \pm 0.54$ & $1.79 \pm 0.30$ & $<0.35$ \\
\hline 236 & 0 & 43 & 21.0 & 41 & 17 & 49 & 5 & 1 & 114.2 & $16.60 \pm 0.94$ & $2.17 \pm 0.42$ & $12.21 \pm 0.81$ & $2.95 \pm 0.37$ & $3.50 \pm 0.44$ \\
\hline 237 & 0 & 43 & 23.0 & 41 & 31 & 47 & 8 & 1 & 35.7 & $1.68 \pm 0.33$ & $<0.52$ & $1.22 \pm 0.24$ & $<0.43$ & $0.98 \pm 0.21$ \\
\hline 238 & 0 & 43 & 23.8 & 41 & 14 & 19 & 10 & 1 & 10.5 & $<1.26$ & $<0.26$ & $0.72 \pm 0.21$ & $<0.75$ & $<0.42$ \\
\hline 239 & 0 & 43 & 25.9 & 41 & 53 & 12 & 14 & 1 & 10.3 & $<1.47$ & $<0.51$ & $<1.45$ & $<0.26$ & $1.07 \pm 0.30$ \\
\hline 240 & 0 & 43 & 26.6 & 41 & 26 & 14 & 7 & 1 & 218.4 & $5.87 \pm 0.52$ & $<0.39$ & $4.78 \pm 0.43$ & $1.26 \pm 0.23$ & $3.53 \pm 0.37$ \\
\hline 241 & 0 & 43 & 26.7 & 41 & 18 & 27 & 7 & 1 & 271.8 & $11.96 \pm 0.76$ & $<1.71$ & $7.20 \pm 0.54$ & $5.13 \pm 0.44$ & $1.90 \pm 0.30$ \\
\hline 242 & 0 & 43 & 28.4 & 41 & 7 & 48 & 6 & 1 & 281.2 & $5.97 \pm 0.52$ & $<0.32$ & $5.60 \pm 0.47$ & $1.25 \pm 0.24$ & $4.42 \pm 0.41$ \\
\hline 243 & 0 & 43 & 28.4 & 42 & 22 & 12 & 14 & 4 & 24.5 & $<0.21$ & $<0.15$ & $2.46 \pm 0.44$ & $<1.29$ & $1.88 \pm 0.36$ \\
\hline 244 & 0 & 43 & 31.7 & 41 & 10 & 37 & 7 & 1 & 78.0 & $2.44 \pm 0.36$ & $<0.43$ & $1.88 \pm 0.28$ & $<0.31$ & $1.71 \pm 0.26$ \\
\hline 245 & 0 & 43 & 32.3 & 42 & 4 & 58 & 12 & 1 & 10.0 & $<1.91$ & $<0.77$ & $<1.59$ & $<0.37$ & $0.91 \pm 0.35$ \\
\hline 246 & 0 & 43 & 32.9 & 41 & 16 & 14 & 12 & 1 & 39.9 & $10.76 \pm 1.01$ & $3.58 \pm 0.50$ & $<2.20$ & $<0.71$ & $<0.43$ \\
\hline 247 & 0 & 43 & 33.9 & 41 & 13 & 25 & 6 & 1 & 1258.7 & $21.31 \pm 0.90$ & $<1.69$ & $17.98 \pm 0.79$ & $6.52 \pm 0.48$ & $11.20 \pm 0.62$ \\
\hline 248 & 0 & 43 & 34.4 & 40 & 56 & 30 & 7 & 1 & 219.6 & $6.06 \pm 0.59$ & $<0.91$ & $4.62 \pm 0.47$ & $2.40 \pm 0.34$ & $2.21 \pm 0.32$ \\
\hline 249 & 0 & 43 & 36.7 & 41 & 14 & 42 & 6 & 1 & 1048.8 & $19.14 \pm 0.87$ & $4.72 \pm 0.71$ & $15.26 \pm 0.74$ & $4.94 \pm 0.42$ & $10.03 \pm 0.60$ \\
\hline 250 & 0 & 43 & 38.7 & 41 & 26 & 52 & 8 & 1 & 78.0 & $3.53 \pm 0.44$ & $<0.61$ & $2.57 \pm 0.33$ & $1.68 \pm 0.26$ & $0.80 \pm 0.20$ \\
\hline 251 & 0 & 43 & 38.7 & 42 & 1 & 26 & 10 & 1 & 21.8 & $1.81 \pm 0.53$ & $<0.58$ & $1.63 \pm 0.40$ & $<0.69$ & $1.21 \pm 0.33$ \\
\hline 252 & 0 & 43 & 40.3 & 40 & 54 & 36 & 9 & 1 & 55.3 & $4.08 \pm 0.59$ & $<2.45$ & $2.38 \pm 0.38$ & $<1.20$ & $1.42 \pm 0.28$ \\
\hline 253 & 0 & 43 & 41.6 & 41 & 53 & 13 & 11 & 1 & 15.5 & $<1.41$ & $<0.42$ & $1.09 \pm 0.29$ & $0.72 \pm 0.22$ & $<0.42$ \\
\hline 254 & 0 & 43 & 43.0 & 41 & 28 & 52 & 8 & 1 & 48.0 & $2.54 \pm 0.39$ & $<0.58$ & $1.92 \pm 0.30$ & $<0.72$ & $1.40 \pm 0.25$ \\
\hline 255 & 0 & 43 & 43.1 & 41 & 12 & 27 & 8 & 1 & 33.4 & $1.82 \pm 0.35$ & $<0.27$ & $1.40 \pm 0.26$ & $0.69 \pm 0.18$ & $0.68 \pm 0.19$ \\
\hline 256 & 0 & 43 & 44.1 & 41 & 24 & 8 & 9 & 1 & 60.0 & $3.72 \pm 0.50$ & $<0.36$ & $2.55 \pm 0.35$ & $1.43 \pm 0.30$ & $1.35 \pm 0.25$ \\
\hline 257 & 0 & 43 & 44.9 & 41 & 37 & 0 & 8 & 1 & 49.7 & $2.16 \pm 0.36$ & $<0.46$ & $1.52 \pm 0.26$ & $0.50 \pm 0.16$ & $1.00 \pm 0.21$ \\
\hline 258 & 0 & 43 & 48.6 & 41 & 27 & 46 & 48 & 1 & 10.0 & $<1.98$ & $<0.47$ & $<0.97$ & $<0.27$ & $0.51 \pm 0.17$ \\
\hline 259 & 0 & 43 & 49.7 & 40 & 49 & 49 & 12 & 1 & 15.8 & $<1.74$ & $<0.43$ & $1.37 \pm 0.37$ & $<0.70$ & $<1.03$ \\
\hline 260 & 0 & 43 & 52.5 & 40 & 16 & 29 & 20 & 4 & 10.4 & $<0.13$ & $<0.13$ & $<1.95$ & $<0.59$ & $<1.37$ \\
\hline 261 & 0 & 43 & 53.1 & 41 & 11 & 53 & 11 & 1 & 10.3 & $<1.20$ & $<0.56$ & $<0.72$ & $0.56 \pm 0.18$ & $<0.13$ \\
\hline 262 & 0 & 43 & 53.2 & 41 & 16 & 54 & 6 & 1 & 1565.6 & $23.50 \pm 0.96$ & $1.32 \pm 0.32$ & $20.69 \pm 0.88$ & $7.17 \pm 0.51$ & $13.57 \pm 0.71$ \\
\hline 263 & 0 & 43 & 53.7 & 41 & 6 & 13 & 9 & 1 & 25.6 & $1.36 \pm 0.34$ & $<0.43$ & $1.15 \pm 0.26$ & $0.55 \pm 0.17$ & $0.57 \pm 0.18$ \\
\hline
\end{tabular}


Table 5. continued.

\begin{tabular}{|c|c|c|c|c|c|c|c|c|c|c|c|c|c|c|}
\hline $\begin{array}{l}S I I \\
\text { No. } \\
(1)\end{array}$ & $\begin{array}{l}\text { I } \\
(\mathrm{h}) \\
(2)\end{array}$ & $\begin{array}{l}\text { A } \\
(\mathrm{m}) \\
(3)\end{array}$ & $\begin{array}{l}(\mathrm{J} 2 \mathrm{C} \\
(\mathrm{s}) \\
(4)\end{array}$ & $\begin{array}{l}00) \\
\left(\begin{array}{l}0 \\
(5)\end{array}\right. \\
(5)\end{array}$ & $\begin{array}{c}\left({ }^{\prime}\right) \\
(6)\end{array}$ & $\begin{array}{l}\mathrm{C} \\
\left({ }^{\prime \prime}\right) \\
(7)\end{array}$ & $\begin{array}{r}\sigma_{\text {Pos }} \\
\left({ }^{\prime \prime}\right) \\
(8)\end{array}$ & $\begin{array}{l}\text { Cl. } \\
\text { (9) }\end{array}$ & $\begin{array}{c}\text { Maxlik } \\
(\mathrm{LH}) \\
(10)\end{array}$ & $\begin{array}{c}\text { Rate }(B) \\
\left(\mathrm{ct} \cdot \mathrm{ks}^{-1}\right) \\
(11)\end{array}$ & $\begin{array}{c}\text { Rate }(S) \\
\left(\mathrm{ct} \cdot \mathrm{ks}^{-1}\right) \\
(12)\end{array}$ & $\begin{array}{c}\text { Rate }(H) \\
\left(\mathrm{ct} \cdot \mathrm{ks}^{-1}\right) \\
(13)\end{array}$ & $\begin{array}{c}\text { Rate }\left(H_{1}\right) \\
\left(\mathrm{ct} \cdot \mathrm{ks}^{-1}\right) \\
(14)\end{array}$ & $\begin{array}{c}\text { Rate }\left(H_{2}\right) \\
\left(\mathrm{ct} \cdot \mathrm{ks}^{-1}\right) \\
(15)\end{array}$ \\
\hline 264 & 0 & 43 & 54.6 & 40 & 45 & 38 & 9 & 1 & 53.9 & $3.17 \pm 0.70$ & $<0.61$ & $3.36 \pm 0.62$ & $0.91 \pm 0.34$ & $2.40 \pm 0.52$ \\
\hline 265 & 0 & 43 & 54.8 & 41 & 52 & 53 & 9 & 1 & 24.3 & $1.40 \pm 0.37$ & $<0.54$ & $1.03 \pm 0.25$ & $0.63 \pm 0.19$ & $<0.55$ \\
\hline 266 & 0 & 43 & 55.6 & 41 & 22 & 2 & 8 & 1 & 32.6 & $2.07 \pm 0.36$ & $<0.74$ & $1.19 \pm 0.25$ & $<0.40$ & $0.90 \pm 0.21$ \\
\hline 267 & 0 & 43 & 57.4 & 41 & 27 & 25 & 9 & 1 & 22.2 & $1.42 \pm 0.31$ & $<0.71$ & $0.95 \pm 0.22$ & $<0.41$ & $0.64 \pm 0.17$ \\
\hline 268 & 0 & 43 & 57.6 & 41 & 30 & 53 & 8 & 1 & 31.5 & $1.41 \pm 0.30$ & $<0.42$ & $1.03 \pm 0.21$ & $<0.42$ & $0.73 \pm 0.17$ \\
\hline 269 & 0 & 43 & 57.8 & 41 & 13 & 45 & 11 & 1 & 10.4 & $<0.79$ & $<0.21$ & $<0.72$ & $0.46 \pm 0.15$ & $<0.22$ \\
\hline 270 & 0 & 43 & 58.8 & 41 & 57 & 17 & 8 & 1 & 28.3 & $<1.35$ & $<0.28$ & $0.97 \pm 0.26$ & $<0.20$ & $0.88 \pm 0.23$ \\
\hline 271 & 0 & 44 & 0.9 & 40 & 56 & 13 & 10 & 1 & 52.8 & $2.73 \pm 0.55$ & $<0.82$ & $2.81 \pm 0.46$ & $0.77 \pm 0.24$ & $1.82 \pm 0.34$ \\
\hline 272 & 0 & 44 & 4.3 & 41 & 49 & 5 & 12 & 1 & 10.2 & $<1.33$ & $<0.43$ & $0.82 \pm 0.24$ & $<0.68$ & $<0.46$ \\
\hline 273 & 0 & 44 & 4.5 & 41 & 21 & 18 & 9 & 1 & 16.7 & $<1.15$ & $<0.46$ & $<0.71$ & $<0.11$ & $0.61 \pm 0.17$ \\
\hline 274 & 0 & 44 & 5.8 & 41 & 52 & 1 & 10 & 1 & 17.2 & $<1.41$ & $<1.10$ & $0.67 \pm 0.21$ & $<0.14$ & $0.67 \pm 0.19$ \\
\hline 275 & 0 & 44 & 7.8 & 41 & 56 & 7 & 9 & 1 & 20.6 & $<1.41$ & $<0.52$ & $0.92 \pm 0.25$ & $<0.47$ & $0.73 \pm 0.21$ \\
\hline 276 & 0 & 44 & 10.2 & 42 & 5 & 5 & 10 & 1 & 15.4 & $1.74 \pm 0.48$ & $<0.81$ & $1.10 \pm 0.32$ & $<0.57$ & $0.76 \pm 0.26$ \\
\hline 277 & 0 & 44 & 12.3 & 41 & 31 & 39 & 11 & 1 & 12.3 & $<0.67$ & $<0.13$ & $0.62 \pm 0.18$ & $<0.20$ & $0.53 \pm 0.16$ \\
\hline 278 & 0 & 44 & 13.7 & 42 & 8 & 44 & 11 & 1 & 10.1 & $<1.46$ & $<0.66$ & $<1.08$ & $<0.82$ & $<0.91$ \\
\hline 279 & 0 & 44 & 13.8 & 41 & 57 & 51 & 9 & 1 & 14.3 & $<0.99$ & $<0.56$ & $<0.60$ & $<0.10$ & $0.54 \pm 0.18$ \\
\hline 280 & 0 & 44 & 13.8 & 42 & 14 & 4 & 11 & 1 & 17.4 & $<3.01$ & $<1.14$ & $1.48 \pm 0.47$ & $<0.53$ & $1.35 \pm 0.41$ \\
\hline 281 & 0 & 44 & 14.9 & 42 & 6 & 34 & 12 & 1 & 12.0 & $<1.88$ & $<0.62$ & $1.25 \pm 0.36$ & $<0.51$ & $1.00 \pm 0.31$ \\
\hline 282 & 0 & 44 & 15.3 & 40 & 26 & 50 & 20 & 4 & 23.5 & $<0.48$ & $<0.10$ & $2.30 \pm 0.41$ & $1.28 \pm 0.30$ & $1.18 \pm 0.31$ \\
\hline 283 & 0 & 44 & 15.9 & 41 & 26 & 22 & 10 & 1 & 14.3 & $1.22 \pm 0.30$ & $<0.75$ & $0.60 \pm 0.18$ & $<0.37$ & $<0.48$ \\
\hline 284 & 0 & 44 & 16.4 & 41 & 11 & 14 & 10 & 1 & 12.1 & $<0.90$ & $<0.36$ & $<0.68$ & $<0.09$ & $0.46 \pm 0.16$ \\
\hline 285 & 0 & 44 & 20.2 & 41 & 34 & 12 & 10 & 1 & 10.2 & $0.98 \pm 0.28$ & $<0.74$ & $<0.63$ & $<0.22$ & $<0.44$ \\
\hline 286 & 0 & 44 & 22.3 & 41 & 45 & 7 & 8 & 1 & 86.4 & $3.07 \pm 0.42$ & $<0.60$ & $2.40 \pm 0.32$ & $0.73 \pm 0.18$ & $1.65 \pm 0.26$ \\
\hline 287 & 0 & 44 & 23.7 & 42 & 0 & 15 & 9 & 1 & 12.5 & $<1.35$ & $<0.69$ & $0.67 \pm 0.21$ & $<0.41$ & $<0.55$ \\
\hline 288 & 0 & 44 & 25.0 & 41 & 31 & 55 & 9 & 1 & 31.7 & $1.54 \pm 0.32$ & $<0.40$ & $1.24 \pm 0.24$ & $<0.62$ & $0.74 \pm 0.18$ \\
\hline 289 & 0 & 44 & 25.6 & 41 & 36 & 30 & 8 & 1 & 42.1 & $1.86 \pm 0.34$ & $<0.48$ & $1.33 \pm 0.24$ & $0.64 \pm 0.17$ & $0.70 \pm 0.17$ \\
\hline 290 & 0 & 44 & 29.2 & 41 & 21 & 34 & 6 & 1 & 1795.5 & $25.10 \pm 1.02$ & $1.10 \pm 0.31$ & $22.54 \pm 0.94$ & $6.86 \pm 0.52$ & $15.71 \pm 0.78$ \\
\hline 291 & 0 & 44 & 29.5 & 41 & 15 & 31 & 14 & 1 & 11.2 & $<1.92$ & $<0.41$ & $1.08 \pm 0.29$ & $<0.98$ & $<0.43$ \\
\hline 292 & 0 & 44 & 31.8 & 42 & 7 & 31 & 10 & 1 & 10.2 & $<1.47$ & $<0.81$ & $<0.78$ & $<0.23$ & $0.49 \pm 0.18$ \\
\hline 293 & 0 & 44 & 35.7 & 42 & 10 & 47 & 12 & 1 & 10.1 & $<1.52$ & $<0.62$ & $<1.18$ & $<0.46$ & $<0.86$ \\
\hline 294 & 0 & 44 & 36.7 & 41 & 45 & 8 & 10 & 1 & 13.6 & $<0.79$ & $<0.36$ & $<0.82$ & $<0.15$ & $0.51 \pm 0.15$ \\
\hline 295 & 0 & 44 & 42.0 & 41 & 13 & 42 & 10 & 1 & 11.2 & $<1.05$ & $<0.60$ & $<0.64$ & $<0.14$ & $0.51 \pm 0.18$ \\
\hline 296 & 0 & 44 & 45.5 & 42 & 43 & 2 & 15 & 4 & 38.9 & $<2.03$ & $<0.38$ & $6.19 \pm 0.91$ & $<3.03$ & $3.96 \pm 0.67$ \\
\hline 297 & 0 & 44 & 47.5 & 41 & 22 & 52 & 8 & 1 & 36.7 & $1.51 \pm 0.33$ & $<0.35$ & $1.24 \pm 0.25$ & $0.48 \pm 0.16$ & $0.74 \pm 0.19$ \\
\hline 298 & 0 & 44 & 48.2 & 42 & 29 & 54 & 12 & 1 & 11.2 & $<5.17$ & $<1.95$ & $<4.17$ & $2.83 \pm 1.01$ & $<0.67$ \\
\hline 299 & 0 & 44 & 50.2 & 41 & 28 & 59 & 9 & 1 & 33.4 & $1.53 \pm 0.32$ & $<0.33$ & $1.23 \pm 0.24$ & $0.47 \pm 0.15$ & $0.70 \pm 0.18$ \\
\hline 300 & 0 & 44 & 52.0 & 41 & 17 & 11 & 10 & 1 & 13.5 & $<1.01$ & $<0.30$ & $0.60 \pm 0.19$ & $<0.29$ & $0.41 \pm 0.15$ \\
\hline 301 & 0 & 44 & 52.0 & 41 & 27 & 17 & 7 & 1 & 116.2 & $2.94 \pm 0.38$ & $<0.36$ & $2.24 \pm 0.30$ & $0.41 \pm 0.14$ & $1.79 \pm 0.26$ \\
\hline 302 & 0 & 44 & 52.0 & 42 & 25 & 10 & 9 & 1 & 24.3 & $4.85 \pm 1.11$ & $<1.41$ & $3.04 \pm 0.77$ & $<1.72$ & $1.91 \pm 0.60$ \\
\hline 303 & 0 & 44 & 55.1 & 41 & 34 & 38 & 10 & 1 & 16.5 & $<1.12$ & $<0.42$ & $0.54 \pm 0.17$ & $<0.14$ & $0.55 \pm 0.16$ \\
\hline 304 & 0 & 44 & 55.9 & 41 & 59 & 34 & 7 & 1 & 102.4 & $4.71 \pm 0.51$ & $2.15 \pm 0.42$ & $2.46 \pm 0.33$ & $1.28 \pm 0.24$ & $1.16 \pm 0.22$ \\
\hline 305 & 0 & 44 & 56.5 & 40 & 59 & 11 & 8 & 1 & 58.1 & $3.12 \pm 0.65$ & $<0.56$ & $2.78 \pm 0.53$ & $1.11 \pm 0.34$ & $1.64 \pm 0.41$ \\
\hline 306 & 0 & 44 & 57.4 & 41 & 23 & 38 & 7 & 1 & 62.2 & $1.80 \pm 0.34$ & $<0.22$ & $1.66 \pm 0.28$ & $0.48 \pm 0.16$ & $1.18 \pm 0.23$ \\
\hline 307 & 0 & 45 & 0.4 & 41 & 14 & 38 & 10 & 1 & 11.9 & $<1.50$ & $<0.58$ & $0.63 \pm 0.21$ & $<0.25$ & $0.46 \pm 0.17$ \\
\hline 308 & 0 & 45 & 0.8 & 41 & 26 & 48 & 10 & 1 & 11.2 & $<0.87$ & $<0.25$ & $0.52 \pm 0.17$ & $<0.37$ & $<0.38$ \\
\hline 309 & 0 & 45 & 8.5 & 42 & 15 & 42 & 7 & 1 & 176.6 & $6.62 \pm 0.74$ & $<0.79$ & $5.57 \pm 0.61$ & $2.39 \pm 0.42$ & $3.19 \pm 0.46$ \\
\hline 310 & 0 & 45 & 9.2 & 42 & 2 & 31 & 9 & 1 & 22.5 & $1.23 \pm 0.31$ & $<0.49$ & $0.84 \pm 0.20$ & $0.58 \pm 0.17$ & $<0.43$ \\
\hline 311 & 0 & 45 & 10.1 & 41 & 0 & 15 & 12 & 1 & 12.9 & $2.17 \pm 0.62$ & $<1.76$ & $1.05 \pm 0.37$ & $<0.53$ & $0.67 \pm 0.28$ \\
\hline 312 & 0 & 45 & 10.4 & 41 & 45 & 53 & 8 & 1 & 29.1 & $1.59 \pm 0.34$ & $<1.17$ & $1.14 \pm 0.24$ & $<0.79$ & $0.78 \pm 0.18$ \\
\hline 313 & 0 & 45 & 12.3 & 42 & 20 & 26 & 9 & 1 & 16.8 & $<1.83$ & $<0.44$ & $1.18 \pm 0.36$ & $<0.73$ & $0.64 \pm 0.25$ \\
\hline 314 & 0 & 45 & 13.2 & 41 & 36 & 11 & 8 & 1 & 33.7 & $1.49 \pm 0.31$ & $<0.71$ & $1.03 \pm 0.21$ & $0.66 \pm 0.17$ & $0.35 \pm 0.13$ \\
\hline 315 & 0 & 45 & 17.1 & 42 & 17 & 10 & 10 & 1 & 11.0 & $<1.86$ & $<0.98$ & $<0.91$ & $<0.21$ & $0.47 \pm 0.19$ \\
\hline 316 & 0 & 45 & 26.5 & 41 & 32 & 45 & 8 & 1 & 50.7 & $1.81 \pm 0.35$ & $<0.22$ & $1.77 \pm 0.29$ & $0.45 \pm 0.15$ & $1.41 \pm 0.25$ \\
\hline 317 & 0 & 45 & 27.4 & 41 & 39 & 0 & 10 & 1 & 18.6 & $1.57 \pm 0.35$ & $<1.15$ & $0.82 \pm 0.21$ & $<0.32$ & $0.66 \pm 0.18$ \\
\hline 318 & 0 & 45 & 27.4 & 42 & 10 & 56 & 9 & 1 & 27.5 & $<1.69$ & $<0.26$ & $1.18 \pm 0.27$ & $<0.58$ & $0.88 \pm 0.22$ \\
\hline 319 & 0 & 45 & 27.8 & 41 & 29 & 38 & 7 & 1 & 126.7 & $3.68 \pm 0.46$ & $<0.49$ & $2.93 \pm 0.37$ & $0.87 \pm 0.21$ & $2.05 \pm 0.31$ \\
\hline 320 & 0 & 45 & 28.0 & 41 & 20 & 37 & 8 & 1 & 39.3 & $1.57 \pm 0.36$ & $<0.33$ & $1.32 \pm 0.28$ & $<0.39$ & $1.03 \pm 0.24$ \\
\hline 321 & 0 & 45 & 28.3 & 41 & 46 & 4 & 10 & 1 & 11.2 & $<1.21$ & $<1.47$ & $<0.48$ & $<0.11$ & $0.36 \pm 0.13$ \\
\hline 322 & 0 & 45 & 28.7 & 41 & 54 & 6 & 6 & 1 & 2703.3 & $29.63 \pm 0.98$ & $2.87 \pm 0.37$ & $23.80 \pm 0.85$ & $18.78 \pm 0.75$ & $4.91 \pm 0.39$ \\
\hline 323 & 0 & 45 & 30.4 & 42 & 1 & 45 & 11 & 1 & 10.0 & $<0.76$ & $<0.38$ & $<0.50$ & $<0.12$ & $0.37 \pm 0.13$ \\
\hline 324 & 0 & 45 & 31.8 & 42 & 7 & 6 & 8 & 1 & 147.7 & $7.75 \pm 0.69$ & $7.41 \pm 0.66$ & $<0.45$ & $<0.32$ & $<0.18$ \\
\hline 325 & 0 & 45 & 32.6 & 42 & 27 & 48 & 11 & 1 & 13.3 & $<3.12$ & $<3.44$ & $1.30 \pm 0.50$ & $<0.34$ & $1.16 \pm 0.45$ \\
\hline 326 & 0 & 45 & 34.1 & 42 & 41 & 44 & 19 & 4 & 11.2 & $<0.62$ & $<0.28$ & $2.35 \pm 0.63$ & $<0.98$ & $1.69 \pm 0.47$ \\
\hline 327 & 0 & 45 & 34.5 & 42 & 17 & 55 & 9 & 1 & 22.9 & $<1.36$ & $<0.37$ & $0.90 \pm 0.28$ & $<0.20$ & $0.90 \pm 0.26$ \\
\hline 328 & 0 & 45 & 38.1 & 42 & 31 & 53 & 12 & 1 & 10.5 & $4.59 \pm 1.40$ & $<3.50$ & $<3.01$ & $<2.07$ & $<1.30$ \\
\hline 329 & 0 & 45 & 38.2 & 41 & 19 & 30 & 10 & 1 & 21.6 & $<1.57$ & $<0.38$ & $1.12 \pm 0.29$ & $<0.59$ & $0.69 \pm 0.22$ \\
\hline 330 & 0 & 45 & 38.3 & 42 & 12 & 39 & 10 & 1 & 11.8 & $1.43 \pm 0.40$ & $<0.98$ & $0.64 \pm 0.21$ & $<0.25$ & $0.50 \pm 0.18$ \\
\hline 331 & 0 & 45 & 40.2 & 42 & 8 & 5 & 6 & 1 & 1122.7 & $25.83 \pm 1.11$ & $11.23 \pm 0.79$ & $13.77 \pm 0.77$ & $6.70 \pm 0.54$ & $7.01 \pm 0.55$ \\
\hline
\end{tabular}


Table 5. continued.

\begin{tabular}{|c|c|c|c|c|c|c|c|c|c|c|c|c|c|c|}
\hline $\begin{array}{l}S I I \\
\text { No. } \\
(1)\end{array}$ & $\begin{array}{l}{ }^{\mathrm{F}} \\
(\mathrm{h}) \\
(2)\end{array}$ & $\begin{array}{l}\text { A } \\
(\mathrm{m}) \\
(3)\end{array}$ & $\begin{array}{l}(\mathrm{J} 20 \\
(\mathrm{s}) \\
(4)\end{array}$ & $\begin{array}{l}00) \\
\left({ }^{\circ}\right) \\
(5)\end{array}$ & $\begin{array}{l}\left({ }^{\prime}\right) \\
(6)\end{array}$ & $\begin{array}{l}\mathrm{c} \\
\left({ }^{\prime \prime}\right) \\
(7)\end{array}$ & $\begin{array}{r}\sigma_{\text {Pos }} \\
\left({ }^{\prime \prime}\right) \\
(8)\end{array}$ & $\begin{array}{l}\mathrm{Cl} . \\
\text { (9) }\end{array}$ & $\begin{array}{c}\text { Maxlik } \\
(\mathrm{LH}) \\
(10)\end{array}$ & $\begin{array}{c}\text { Rate }(B) \\
\left(\mathrm{ct} \cdot \mathrm{ks}^{-1}\right) \\
(11)\end{array}$ & $\begin{array}{c}\text { Rate }(S) \\
\left(\mathrm{ct} \cdot \mathrm{ks}^{-1}\right) \\
(12)\end{array}$ & $\begin{array}{c}\text { Rate }(H) \\
\left(\mathrm{ct} \cdot \mathrm{ks}^{-1}\right) \\
(13)\end{array}$ & $\begin{array}{c}\operatorname{Rate}\left(H_{1}\right) \\
\left(\mathrm{ct} \cdot \mathrm{ks}^{-1}\right) \\
(14)\end{array}$ & $\begin{array}{c}\text { Rate }\left(H_{2}\right) \\
\left(\mathrm{ct} \cdot \mathrm{ks}^{-1}\right) \\
(15)\end{array}$ \\
\hline 332 & 0 & 45 & 41.1 & 41 & 27 & 49 & 8 & 1 & 34.3 & $1.58 \pm 0.36$ & $<1.21$ & $1.13 \pm 0.25$ & $<0.62$ & $0.67 \pm 0.19$ \\
\hline 333 & 0 & 45 & 42.1 & 41 & 23 & 52 & 9 & 1 & 19.5 & $<1.13$ & $<0.24$ & $0.86 \pm 0.24$ & $<0.66$ & $<0.56$ \\
\hline 334 & 0 & 45 & 42.6 & 42 & 23 & 28 & 10 & 1 & 13.1 & $2.01 \pm 0.60$ & $<1.43$ & $0.93 \pm 0.34$ & $<0.32$ & $0.74 \pm 0.28$ \\
\hline 335 & 0 & 45 & 42.9 & 41 & 20 & 25 & 8 & 1 & 51.6 & $2.18 \pm 0.44$ & $<0.50$ & $1.78 \pm 0.34$ & $0.92 \pm 0.24$ & $0.83 \pm 0.23$ \\
\hline 336 & 0 & 45 & 45.4 & 41 & 39 & 37 & 5 & 1 & 17930 . & $134.43 \pm 1.96$ & $4.71 \pm 0.47$ & $133.47 \pm 2.10$ & $38.77 \pm 1.15$ & $95.71 \pm 1.82$ \\
\hline 337 & 0 & 45 & 55.8 & 41 & 48 & 35 & 10 & 1 & 13.9 & $<0.93$ & $<0.30$ & $0.55 \pm 0.17$ & $<0.23$ & $0.43 \pm 0.14$ \\
\hline 338 & 0 & 45 & 55.9 & 41 & 56 & 33 & 10 & 1 & 22.5 & $1.55 \pm 0.32$ & $1.50 \pm 0.30$ & $<0.21$ & $<0.25$ & $<0.08$ \\
\hline 339 & 0 & 45 & 56.0 & 42 & 12 & 32 & 9 & 1 & 20.2 & $1.39 \pm 0.39$ & $<2.41$ & $0.91 \pm 0.25$ & $<0.28$ & $0.72 \pm 0.21$ \\
\hline 340 & 0 & 45 & 57.6 & 42 & 3 & 10 & 8 & 1 & 65.3 & $3.03 \pm 0.42$ & $<1.50$ & $1.89 \pm 0.28$ & $0.83 \pm 0.19$ & $1.06 \pm 0.21$ \\
\hline 341 & 0 & 45 & 57.6 & 42 & 26 & 42 & 11 & 1 & 12.6 & $<2.94$ & $<1.05$ & $1.43 \pm 0.49$ & $<0.97$ & $0.98 \pm 0.37$ \\
\hline 342 & 0 & 46 & 0.2 & 41 & 33 & 10 & 10 & 1 & 17.5 & $1.06 \pm 0.31$ & $<0.37$ & $0.79 \pm 0.22$ & $<0.46$ & $0.47 \pm 0.16$ \\
\hline 343 & 0 & 46 & 5.9 & 41 & 51 & 37 & 11 & 1 & 10.2 & $<1.34$ & $<0.64$ & $<0.69$ & $<0.14$ & $0.43 \pm 0.14$ \\
\hline 344 & 0 & 46 & 10.9 & 42 & 3 & 53 & 9 & 1 & 40.7 & $1.67 \pm 0.35$ & $<0.37$ & $1.28 \pm 0.25$ & $<0.54$ & $0.88 \pm 0.19$ \\
\hline 345 & 0 & 46 & 11.6 & 41 & 58 & 58 & 10 & 1 & 15.2 & $<1.21$ & $<0.42$ & $0.53 \pm 0.16$ & $<0.15$ & $0.49 \pm 0.14$ \\
\hline 346 & 0 & 46 & 12.0 & 42 & 8 & 27 & 7 & 1 & 80.3 & $2.73 \pm 0.43$ & $<0.50$ & $2.29 \pm 0.34$ & $0.56 \pm 0.18$ & $1.73 \pm 0.29$ \\
\hline 347 & 0 & 46 & 13.3 & 41 & 50 & 37 & 10 & 1 & 11.6 & $<0.99$ & $<0.46$ & $<0.60$ & $<0.54$ & $0.38 \pm 0.13$ \\
\hline 348 & 0 & 46 & 14.8 & 42 & 21 & 34 & 12 & 1 & 11.2 & $<1.24$ & $<0.54$ & $<1.55$ & $<0.13$ & $0.82 \pm 0.29$ \\
\hline 349 & 0 & 46 & 15.7 & 41 & 24 & 9 & 13 & 1 & 11.6 & $<1.29$ & $<0.46$ & $1.00 \pm 0.32$ & $<0.73$ & $<0.55$ \\
\hline 350 & 0 & 46 & 19.9 & 42 & 14 & 36 & 9 & 1 & 13.2 & $<0.89$ & $<0.24$ & $0.70 \pm 0.22$ & $<0.47$ & $<0.53$ \\
\hline 351 & 0 & 46 & 24.2 & 42 & 4 & 38 & 6 & 1 & 1690.2 & $33.17 \pm 1.16$ & $<0.71$ & $29.36 \pm 1.02$ & $6.85 \pm 0.50$ & $22.29 \pm 0.88$ \\
\hline 352 & 0 & 46 & 24.3 & 42 & 9 & 53 & 9 & 1 & 29.4 & $1.79 \pm 0.42$ & $<1.12$ & $2.45 \pm 0.46$ & $<0.48$ & $1.16 \pm 0.27$ \\
\hline 353 & 0 & 46 & 25.6 & 41 & 16 & 30 & 13 & 1 & 16.0 & $2.42 \pm 0.73$ & $<1.76$ & $1.83 \pm 0.54$ & $<1.30$ & $<1.23$ \\
\hline 354 & 0 & 46 & 26.7 & 42 & 1 & 51 & 6 & 1 & 3781.8 & $37.64 \pm 1.15$ & $<1.28$ & $34.53 \pm 1.06$ & $10.53 \pm 0.59$ & $24.08 \pm 0.88$ \\
\hline 355 & 0 & 46 & 31.5 & 42 & 44 & 2 & 16 & 4 & 10.2 & $<1.40$ & $<0.42$ & $1.99 \pm 0.57$ & $<0.74$ & $<1.99$ \\
\hline 356 & 0 & 46 & 34.1 & 42 & 7 & 29 & 10 & 1 & 15.3 & $<1.44$ & $<0.47$ & $0.70 \pm 0.20$ & $<1.24$ & $0.52 \pm 0.17$ \\
\hline 357 & 0 & 46 & 36.6 & 41 & 29 & 0 & 11 & 1 & 12.7 & $<1.45$ & $<0.52$ & $0.80 \pm 0.27$ & $<0.57$ & $<0.60$ \\
\hline 358 & 0 & 46 & 38.0 & 41 & 46 & 17 & 11 & 1 & 11.5 & $<0.92$ & $<0.42$ & $<0.61$ & $<0.12$ & $0.40 \pm 0.14$ \\
\hline 359 & 0 & 46 & 39.6 & 42 & 25 & 16 & 9 & 1 & 18.7 & $<2.49$ & $<0.71$ & $1.63 \pm 0.49$ & $<0.76$ & $1.10 \pm 0.39$ \\
\hline 360 & 0 & 46 & 44.8 & 42 & 30 & 38 & 10 & 1 & 16.7 & $<5.19$ & $<1.39$ & $2.76 \pm 0.84$ & $<2.21$ & $<1.66$ \\
\hline 361 & 0 & 46 & 46.9 & 41 & 49 & 8 & 10 & 1 & 12.6 & $<1.14$ & $<0.47$ & $0.59 \pm 0.19$ & $<0.58$ & $<0.40$ \\
\hline 362 & 0 & 46 & 47.3 & 42 & 8 & 56 & 9 & 1 & 28.9 & $1.60 \pm 0.38$ & $<0.57$ & $1.16 \pm 0.26$ & $<0.43$ & $0.95 \pm 0.23$ \\
\hline 363 & 0 & 46 & 55.1 & 42 & 20 & 47 & 6 & 1 & 730.9 & $23.18 \pm 1.50$ & $<0.77$ & $20.88 \pm 1.33$ & $7.71 \pm 0.81$ & $13.02 \pm 1.05$ \\
\hline 364 & 0 & 46 & 56.8 & 41 & 52 & 33 & 9 & 1 & 33.3 & $0.98 \pm 0.29$ & $<0.27$ & $0.83 \pm 0.21$ & $<0.12$ & $0.87 \pm 0.20$ \\
\hline 365 & 0 & 47 & 0.8 & 41 & 41 & 7 & 10 & 1 & 19.0 & $<1.47$ & $<0.40$ & $0.98 \pm 0.27$ & $0.65 \pm 0.22$ & $<0.46$ \\
\hline 366 & 0 & 47 & 1.0 & 41 & 51 & 24 & 9 & 1 & 23.7 & $1.21 \pm 0.33$ & $<0.48$ & $0.88 \pm 0.22$ & $<0.29$ & $0.70 \pm 0.19$ \\
\hline 367 & 0 & 47 & 3.4 & 41 & 58 & 8 & 11 & 1 & 10.7 & $1.21 \pm 0.34$ & $<1.25$ & $<0.52$ & $<0.46$ & $<0.13$ \\
\hline 368 & 0 & 47 & 3.4 & 42 & 4 & 55 & 8 & 1 & 43.7 & $1.89 \pm 0.37$ & $<0.53$ & $1.39 \pm 0.27$ & $<0.58$ & $1.05 \pm 0.23$ \\
\hline 369 & 0 & 47 & 13.8 & 42 & 2 & 13 & 8 & 1 & 68.9 & $3.67 \pm 0.50$ & $1.47 \pm 0.37$ & $1.93 \pm 0.32$ & $0.83 \pm 0.22$ & $1.10 \pm 0.24$ \\
\hline 370 & 0 & 47 & 14.7 & 42 & 21 & 1 & 11 & 1 & 45.9 & $5.48 \pm 1.11$ & $<0.54$ & $6.54 \pm 0.94$ & $1.81 \pm 0.51$ & $4.09 \pm 0.75$ \\
\hline 371 & 0 & 47 & 15.4 & 41 & 40 & 41 & 9 & 1 & 40.0 & $1.81 \pm 0.45$ & $<0.36$ & $1.94 \pm 0.39$ & $0.70 \pm 0.25$ & $1.18 \pm 0.30$ \\
\hline 372 & 0 & 47 & 16.2 & 41 & 35 & 46 & 12 & 1 & 16.9 & $<1.90$ & $<0.53$ & $1.42 \pm 0.38$ & $<0.78$ & $0.77 \pm 0.27$ \\
\hline 373 & 0 & 47 & 20.1 & 41 & 48 & 37 & 10 & 1 & 18.0 & $1.93 \pm 0.46$ & $<3.26$ & $0.85 \pm 0.25$ & $<0.45$ & $0.60 \pm 0.21$ \\
\hline 374 & 0 & 47 & 25.1 & 42 & 21 & 45 & 10 & 1 & 66.2 & $7.65 \pm 1.29$ & $<0.97$ & $7.22 \pm 1.07$ & $2.49 \pm 0.63$ & $4.10 \pm 0.83$ \\
\hline 375 & 0 & 47 & 26.9 & 41 & 52 & 53 & 9 & 1 & 52.7 & $3.35 \pm 0.54$ & $<0.88$ & $2.49 \pm 0.40$ & $1.00 \pm 0.26$ & $1.48 \pm 0.30$ \\
\hline 376 & 0 & 47 & 27.8 & 41 & 24 & 40 & 13 & 1 & 12.8 & $<5.97$ & $<1.65$ & $3.64 \pm 1.34$ & $<1.07$ & $3.01 \pm 1.23$ \\
\hline 377 & 0 & 47 & 30.6 & 41 & 49 & 24 & 8 & 1 & 81.3 & $4.39 \pm 0.63$ & $<4.52$ & $2.97 \pm 0.46$ & $0.95 \pm 0.27$ & $2.11 \pm 0.38$ \\
\hline 378 & 0 & 47 & 30.9 & 41 & 40 & 42 & 11 & 1 & 10.3 & $<2.17$ & $<1.21$ & $<0.84$ & $<0.52$ & $<0.38$ \\
\hline 379 & 0 & 47 & 31.4 & 41 & 35 & 24 & 12 & 1 & 12.0 & $<1.73$ & $<0.45$ & $1.17 \pm 0.40$ & $<1.00$ & $<0.68$ \\
\hline 380 & 0 & 47 & 40.4 & 42 & 22 & 32 & 5 & 1 & 17.4 & $5.20 \pm 1.48$ & $<1.49$ & $5.80 \pm 1.33$ & $<2.37$ & $<2.68$ \\
\hline 381 & 0 & 47 & 42.8 & 42 & 3 & 2 & 10 & 1 & 12.0 & $<1.20$ & $<0.52$ & $<0.78$ & $<0.15$ & $0.48 \pm 0.18$ \\
\hline 382 & 0 & 47 & 43.2 & 42 & 1 & 19 & 9 & 1 & 24.6 & $1.48 \pm 0.41$ & $<0.54$ & $1.19 \pm 0.30$ & $0.60 \pm 0.21$ & $0.67 \pm 0.23$ \\
\hline 383 & 0 & 47 & 43.8 & 42 & 24 & 16 & 5 & 1 & 17.5 & $<5.17$ & $<1.31$ & $4.65 \pm 1.31$ & $<1.35$ & $<3.45$ \\
\hline 384 & 0 & 47 & 44.5 & 42 & 22 & 37 & 5 & 1 & 15.8 & $<6.19$ & $<1.70$ & $7.36 \pm 1.60$ & $<3.10$ & $<2.63$ \\
\hline 385 & 0 & 47 & 45.0 & 42 & 11 & 3 & 11 & 1 & 10.9 & $<2.25$ & $<1.47$ & $1.22 \pm 0.40$ & $<0.74$ & $0.75 \pm 0.29$ \\
\hline 386 & 0 & 47 & 47.9 & 42 & 19 & 33 & 8 & 1 & 145.6 & $12.77 \pm 1.63$ & $<1.19$ & $12.11 \pm 1.45$ & $2.96 \pm 0.74$ & $8.89 \pm 1.25$ \\
\hline 387 & 0 & 47 & 49.4 & 41 & 53 & 26 & 10 & 1 & 16.9 & $<1.13$ & $<0.31$ & $0.89 \pm 0.28$ & $<0.24$ & $0.78 \pm 0.25$ \\
\hline 388 & 0 & 47 & 49.9 & 41 & 42 & 9 & 11 & 1 & 41.4 & $7.02 \pm 1.15$ & $<1.63$ & $13.74 \pm 1.37$ & $4.06 \pm 0.78$ & $7.97 \pm 1.05$ \\
\hline 389 & 0 & 47 & 51.7 & 42 & 7 & 24 & 9 & 1 & 19.9 & $2.46 \pm 0.59$ & $<3.90$ & $1.23 \pm 0.36$ & $0.80 \pm 0.28$ & $<0.69$ \\
\hline 390 & 0 & 47 & 53.9 & 41 & 35 & 38 & 16 & 1 & 11.8 & $<3.40$ & $3.07 \pm 0.94$ & $<0.45$ & $<0.27$ & $<0.36$ \\
\hline 391 & 0 & 48 & 0.3 & 41 & 40 & 15 & 7 & 1 & 442.6 & $27.75 \pm 2.07$ & $<3.14$ & $25.92 \pm 1.97$ & $10.22 \pm 1.22$ & $16.23 \pm 1.58$ \\
\hline 392 & 0 & 48 & 4.2 & 41 & 56 & 48 & 10 & 1 & 13.1 & $2.11 \pm 0.58$ & $<1.74$ & $<0.85$ & $<0.56$ & $<0.37$ \\
\hline 393 & 0 & 48 & 23.6 & 42 & 10 & 43 & 11 & 1 & 14.8 & $3.61 \pm 1.02$ & $<3.18$ & $1.91 \pm 0.64$ & $<0.45$ & $1.71 \pm 0.58$ \\
\hline 394 & 0 & 48 & 24.6 & 41 & 57 & 18 & 6 & 1 & 614.1 & $35.30 \pm 2.16$ & $21.89 \pm 1.77$ & $14.28 \pm 1.31$ & $8.11 \pm 0.99$ & $6.24 \pm 0.87$ \\
\hline 395 & 0 & 48 & 58.4 & 42 & 23 & 47 & 9 & 4 & 250.2 & $9.21 \pm 0.99$ & $<0.52$ & $15.95 \pm 1.07$ & $6.21 \pm 0.69$ & $9.84 \pm 0.81$ \\
\hline 396 & 0 & 49 & 30.6 & 41 & 58 & 23 & 13 & 1 & 22.1 & $6.87 \pm 2.29$ & $<1.91$ & $6.12 \pm 1.85$ & $<2.66$ & $<5.62$ \\
\hline
\end{tabular}


Table 6. Total list of all ROSAT PSPC X-ray sources in M 31 merged from the source lists of both surveys. The different symbols in front of the RXJ numbers in Col. (1) are explained at the top of the table. The meaning of the different columns is described in Sect. 3.3. The listed count rate errors are purely statistical. The systematic errors are expected to be less than $\sim 15 \%$. Count rates for bulge sources (marked with $\star$ ) may be uncertain due to confusion. For sources not detected in a considered energy band, $1 \sigma$ upper limits have been calculated indicated by a "<"-symbol in front of the upper limit value. A conversion of count rates into fluxes depends on the assumed spectral shape. For M 31-sources a power law with photon index $\Gamma=-2.0$ and $N_{\mathrm{H}}=9 \times 10^{20} \mathrm{~cm}^{-2}$ may be used, leading to a conversion factor $1 \mathrm{ct} \mathrm{ks}^{-1}=3.00 \times 10^{-14}$ erg $\mathrm{cm}^{-2} \mathrm{~s}^{-1}$ in the $0.1-2.0 \mathrm{keV}$ band ( $B$-band). For foreground stars, the application of this conversion factor leads to an overestimation of the flux.

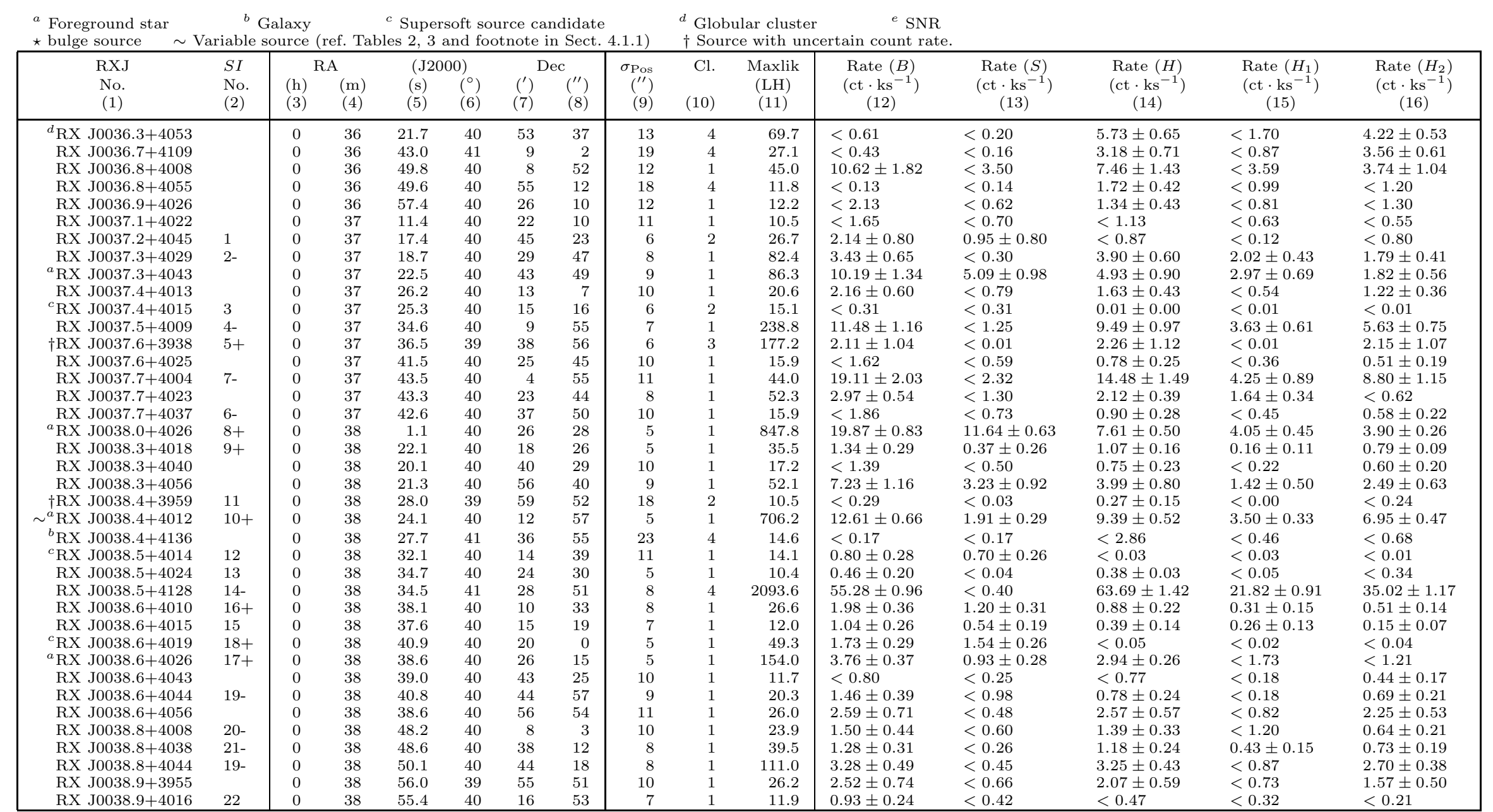




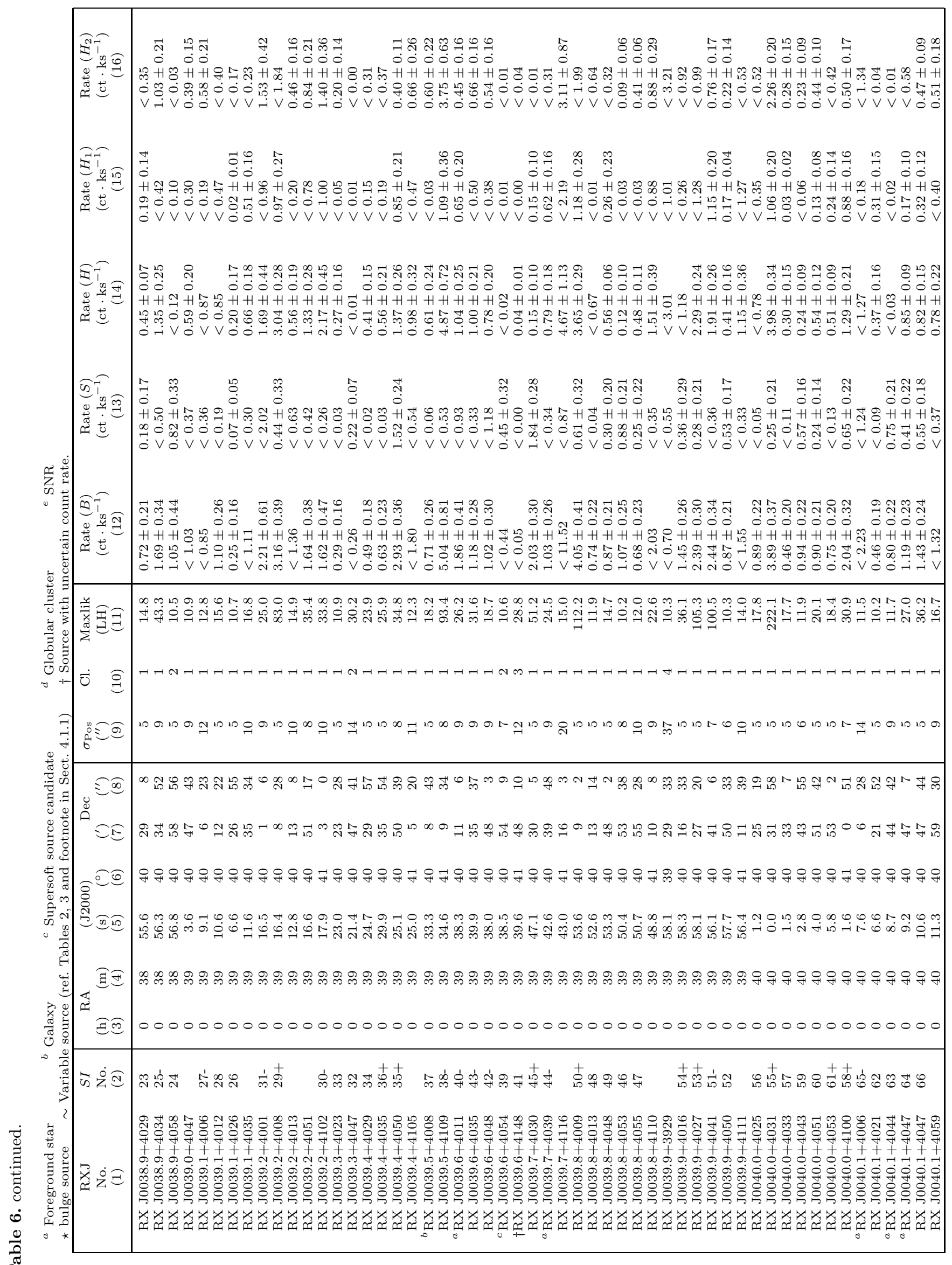




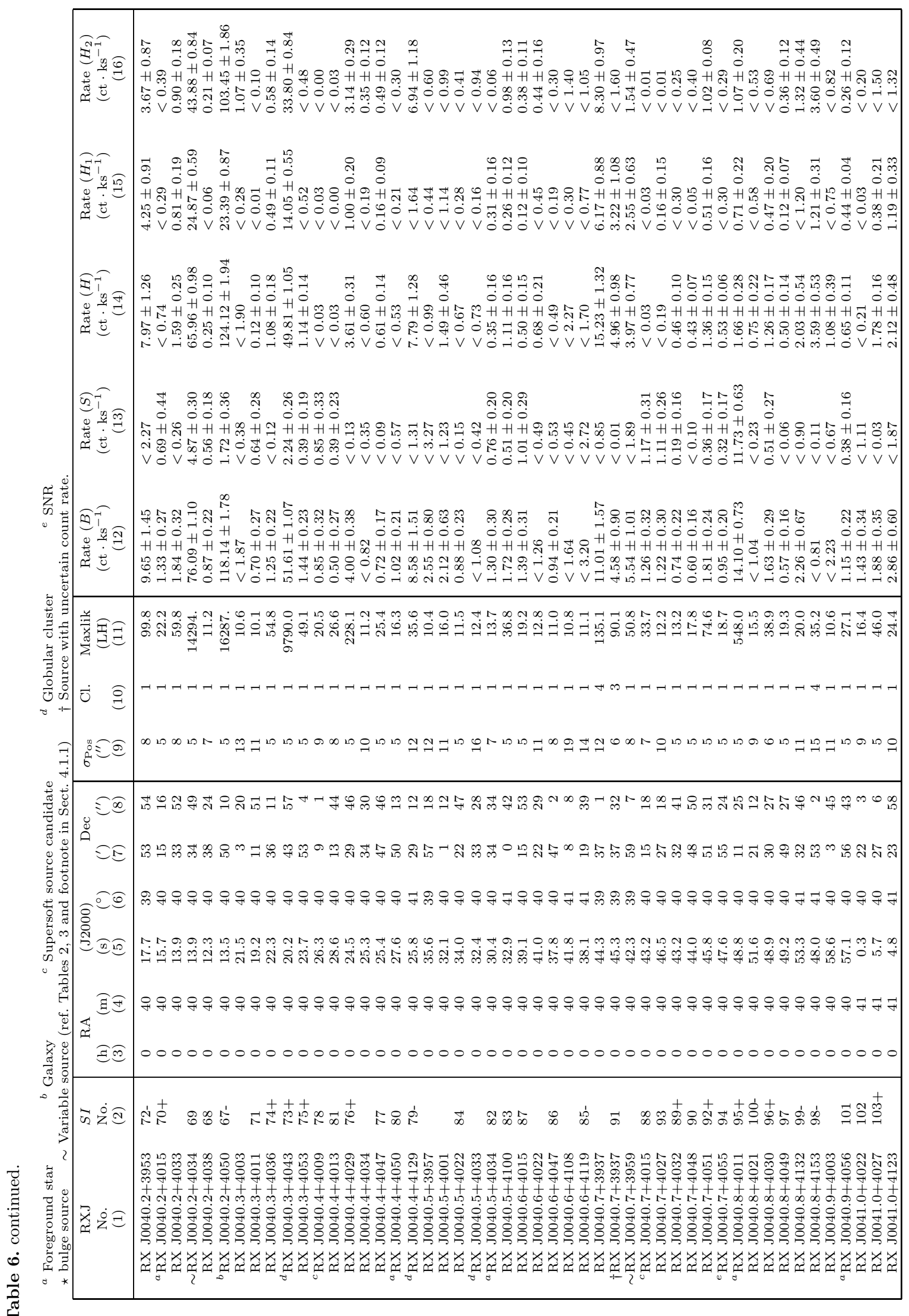




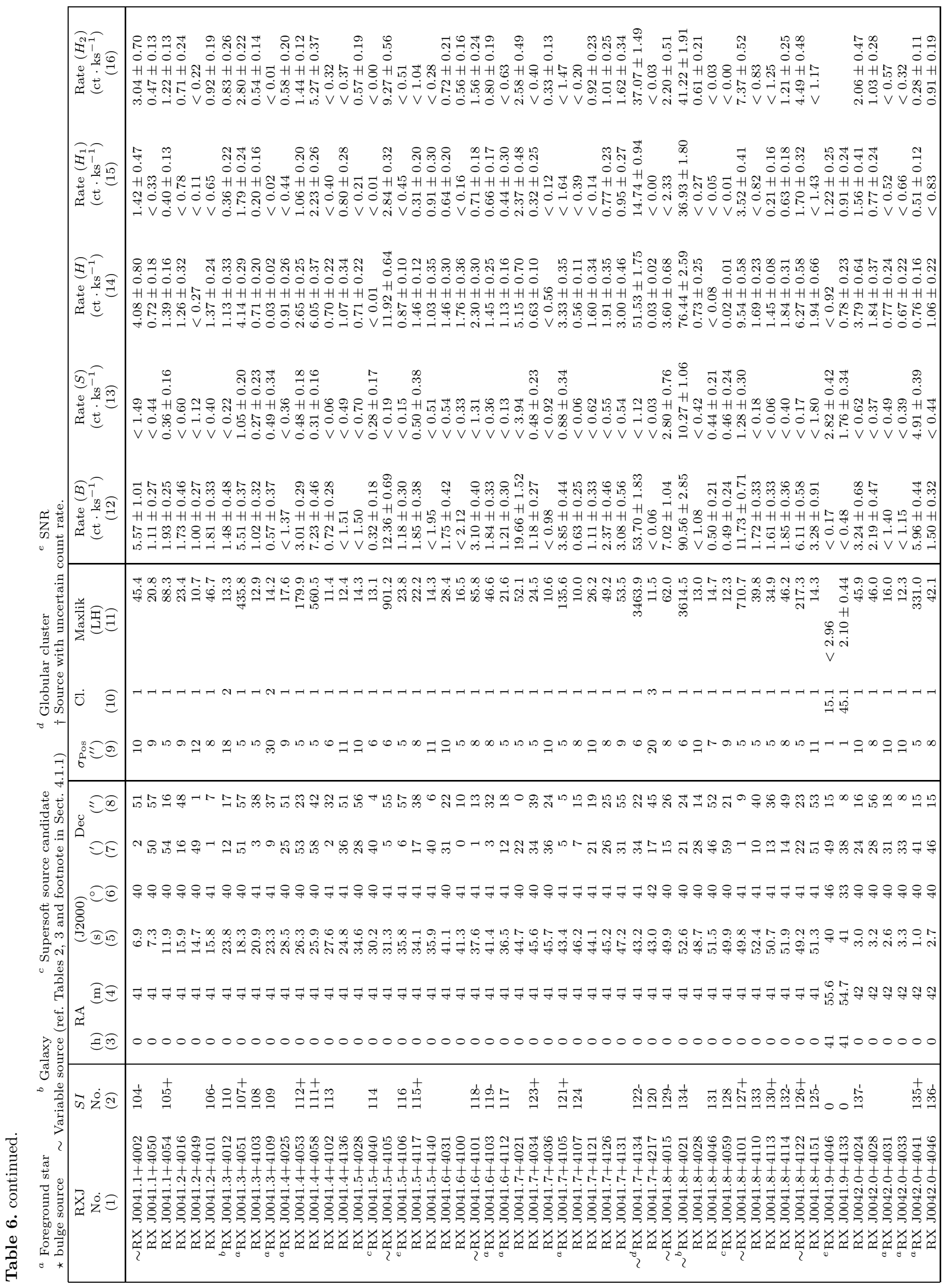




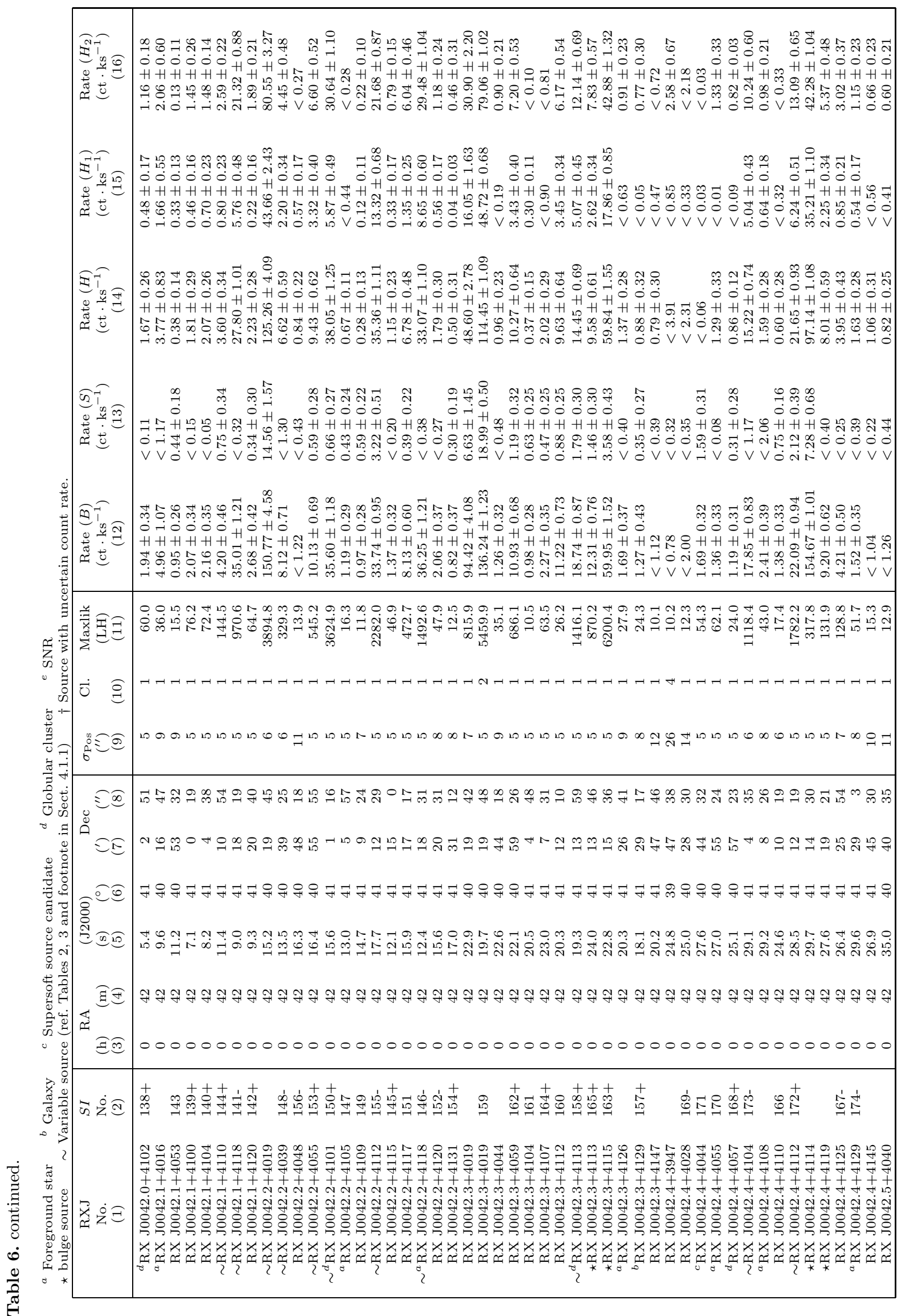




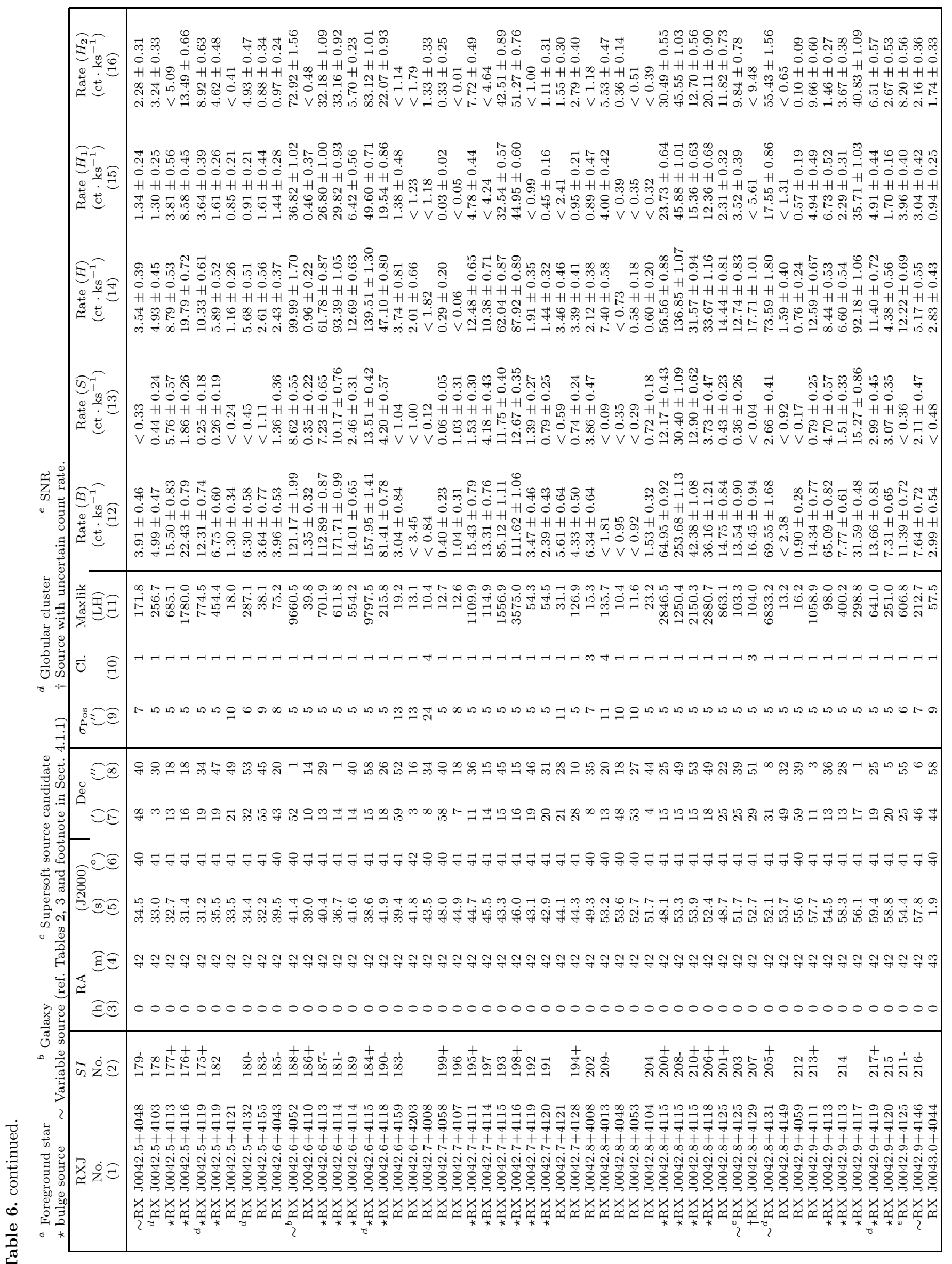




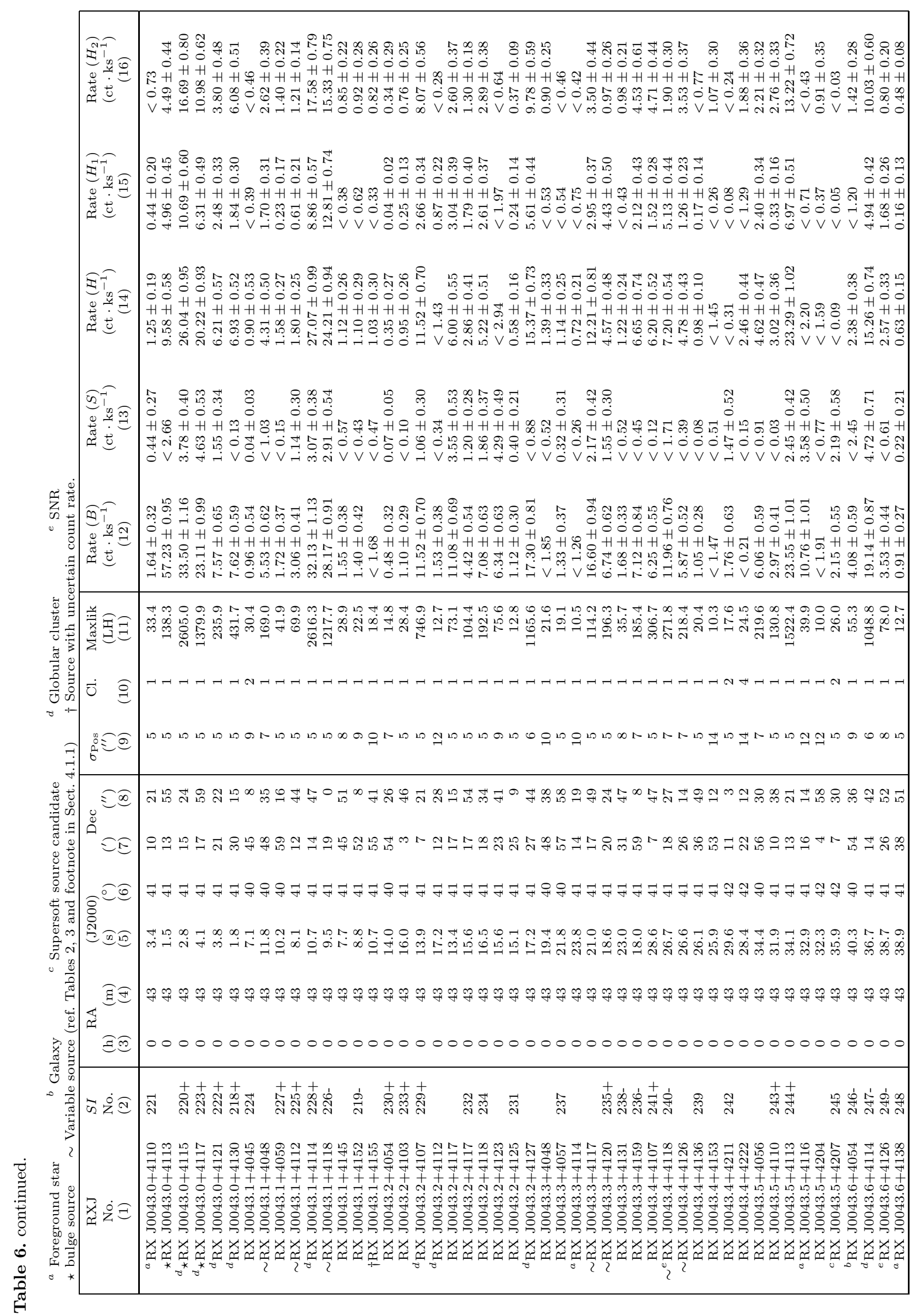




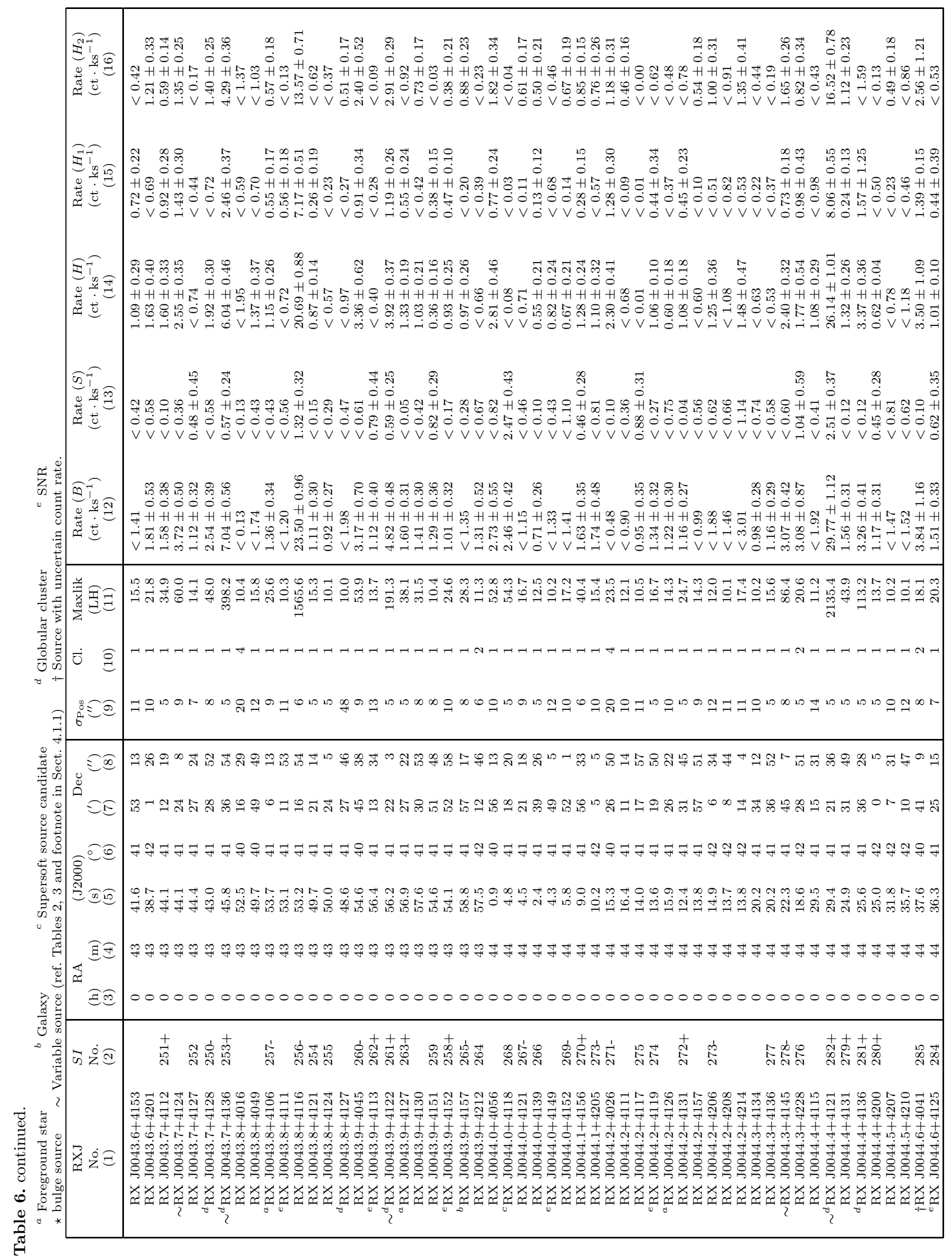




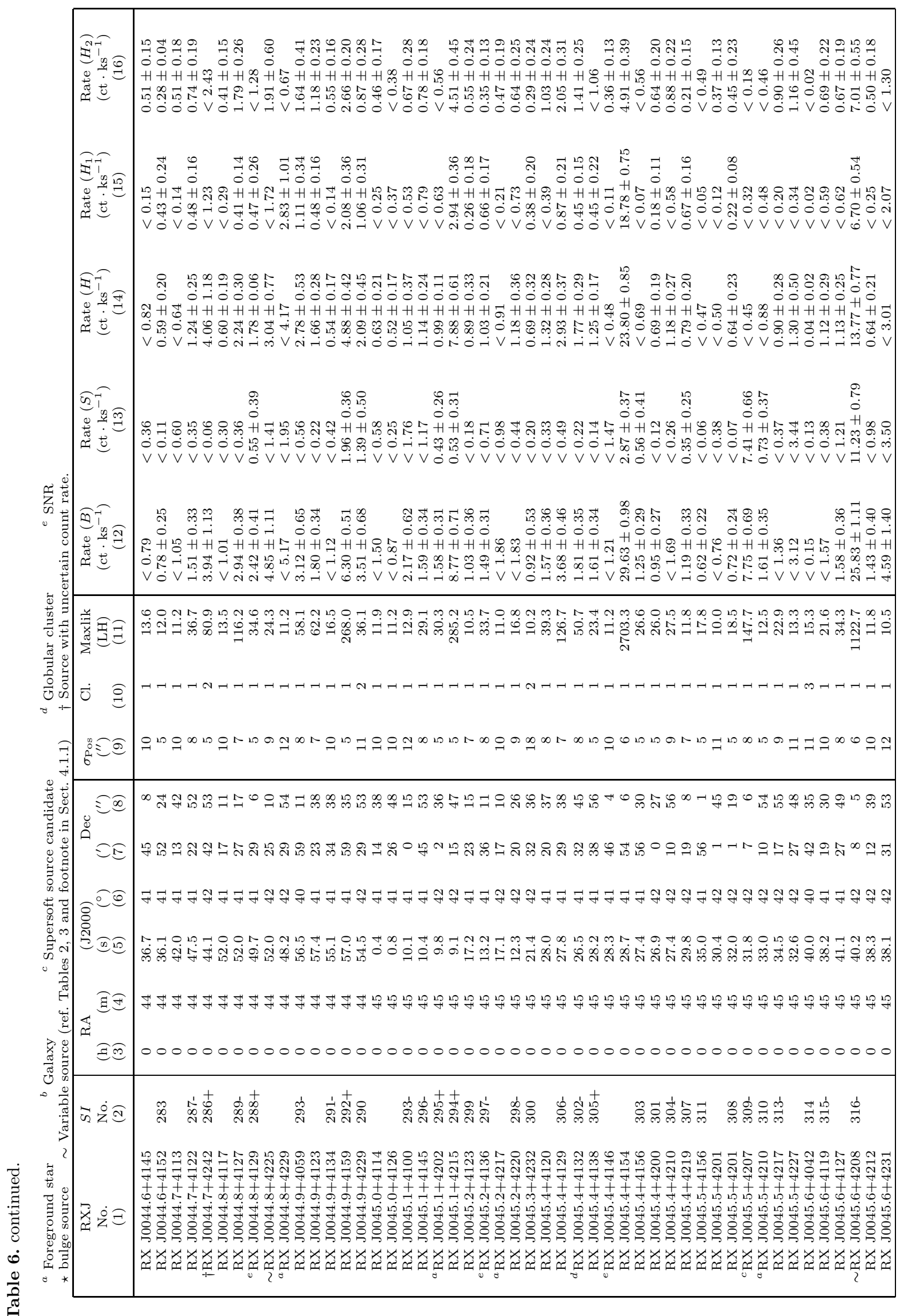




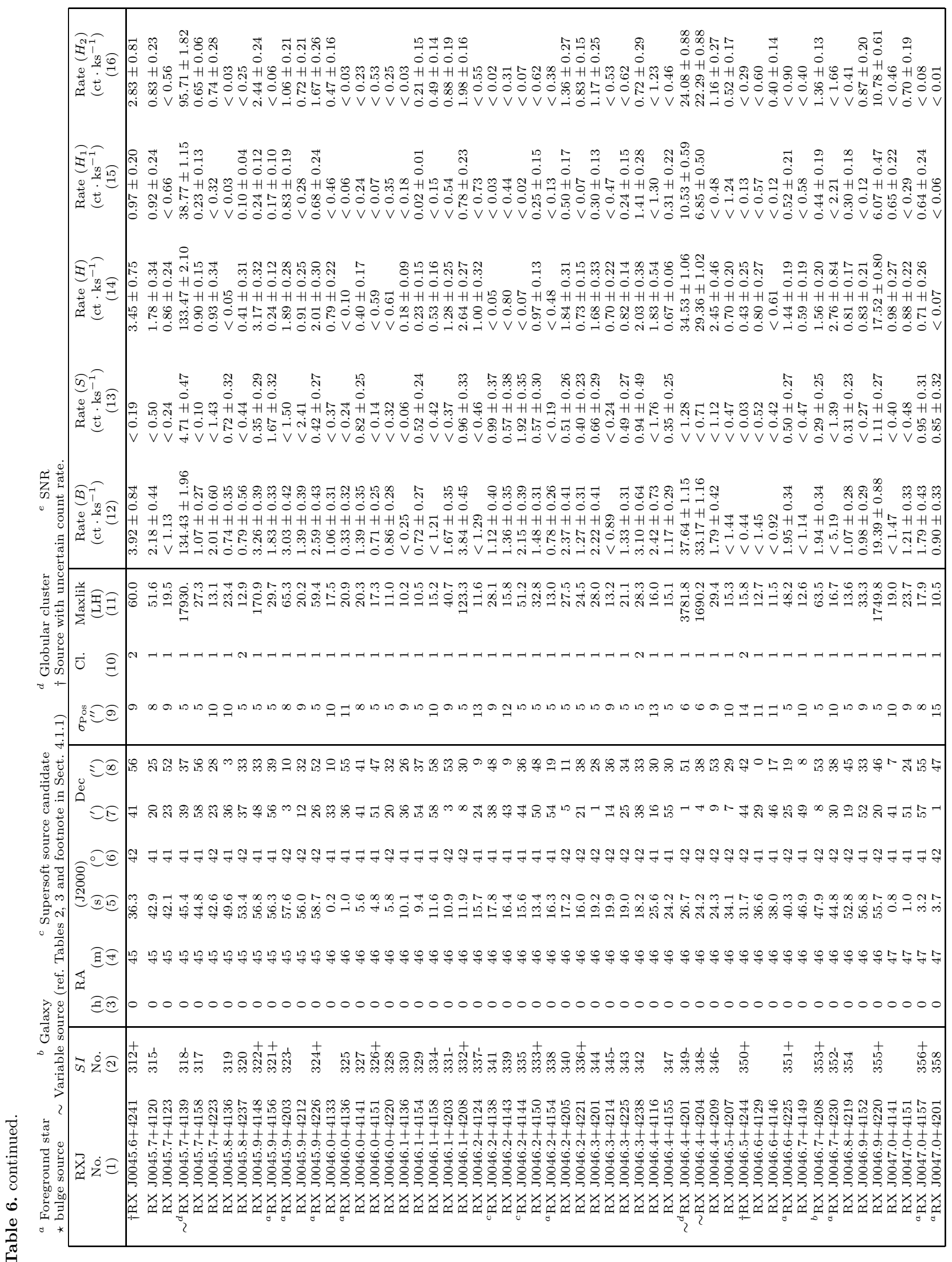




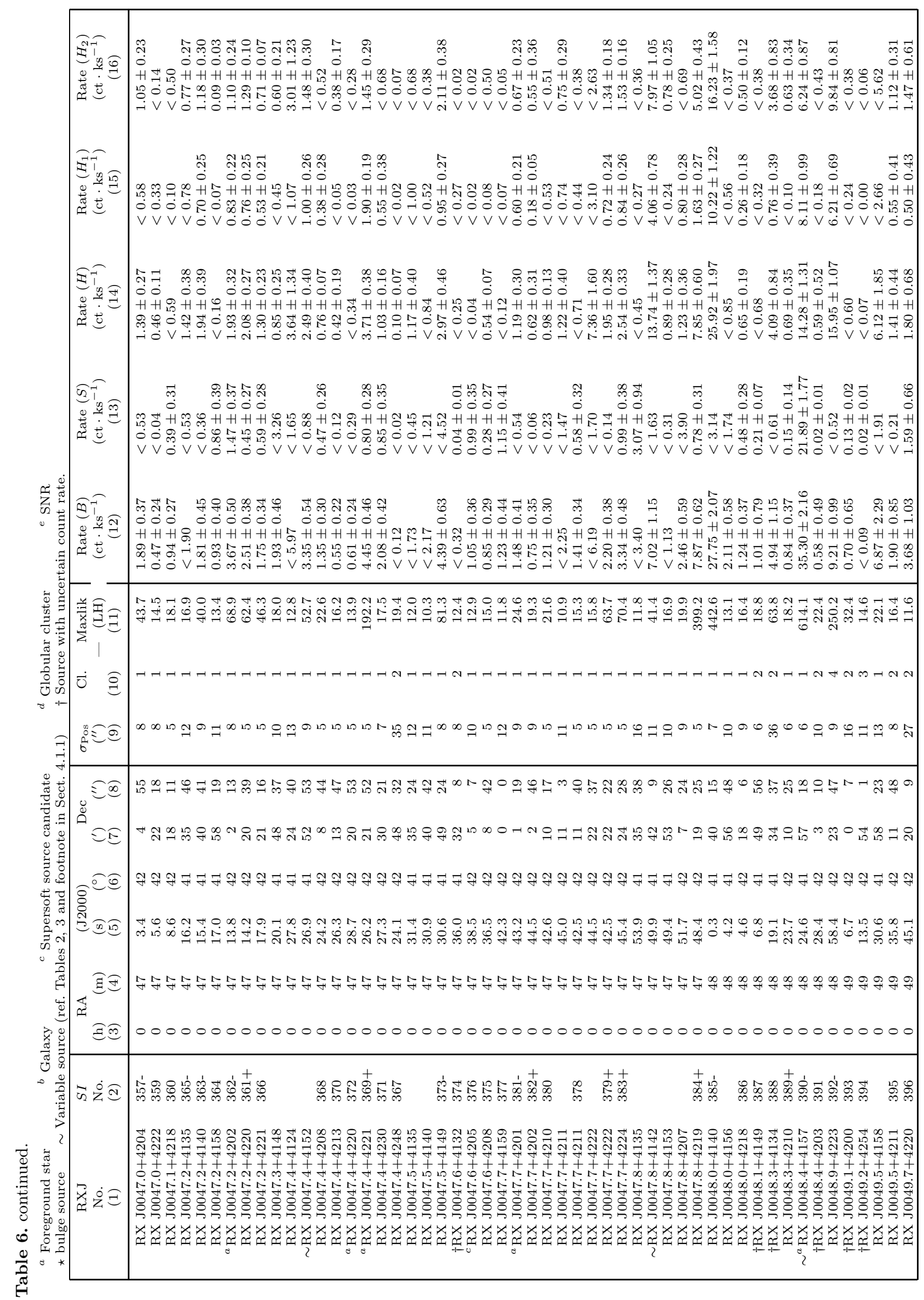


Table 7. Table of the identifications of ROSAT PSPC sources with Einstein sources listed by TF. $F_{\mathrm{R}}$ gives the ROSAT source flux using the Einstein spectral model of $\mathrm{TF}$ and $F_{\mathrm{E}}$ gives the Einstein source flux of the correlated Einstein source (see Sect. 4.1.2). The distance between two correlating sources is given in arcseconds (") as well as in units of the combined positional error $(\sigma)$ of both sources. The last column gives the flux ratio between the ROSAT and the Einstein measurements, showing possible long term variabilities between the epochs of the two observations. Sources with ROSAT numbers preceeded by $\mathrm{a} \star$ belong to the bulge region.

\begin{tabular}{|c|c|c|c|c|c|c|}
\hline $\begin{array}{l}\text { ROSAT } \\
\text { No. }\end{array}$ & $\begin{array}{c}F_{\mathrm{R}}\left(\times 10^{13}\right) \\
(\mathrm{cgs})\end{array}$ & $\begin{array}{l}\text { Einstein } \\
\text { No. }\end{array}$ & $\begin{array}{c}F_{\mathrm{E}}\left(\times 10^{13}\right) \\
(\operatorname{cgs})\end{array}$ & $\left({ }^{\prime \prime}\right)$ & ce $(\sigma)$ & $F_{\mathrm{R}} / F_{\mathrm{E}}$ \\
\hline RX J0039.4+4035 & $0.17 \pm 0.06$ & 1 & $0.60 \pm 0.26$ & 59.1 & 1.31 & $0.28 \pm 0.16$ \\
\hline RX J0040.0+4031 & $1.04 \pm 0.10$ & 2 & $1.64 \pm 0.42$ & 31.7 & 0.70 & $0.63 \pm 0.17$ \\
\hline RX J0040.2+4050 & $31.64 \pm 0.48$ & 3 & $25.38 \pm 2.50$ & 3.6 & 0.59 & $1.25 \pm 0.12$ \\
\hline RX J0040.3+4043 & $13.82 \pm 0.29$ & 4 & $16.35 \pm 2.09$ & 3.4 & 0.58 & $0.85 \pm 0.11$ \\
\hline RX J0040.4+4029 & $1.07 \pm 0.10$ & 5 & $0.99 \pm 0.28$ & 13.4 & 0.30 & $1.08 \pm 0.33$ \\
\hline RX J0040.4+4129 & $2.30 \pm 0.41$ & 6 & $1.06 \pm 0.35$ & 9.5 & 0.20 & $2.17 \pm 0.81$ \\
\hline RX J0040.7+4051 & $0.49 \pm 0.07$ & 7 & $0.66 \pm 0.26$ & 6.0 & 0.13 & $0.74 \pm 0.30$ \\
\hline RX J0041.4+4058 & $1.94 \pm 0.12$ & 8 & $1.77 \pm 0.50$ & 24.6 & 0.54 & $1.09 \pm 0.32$ \\
\hline RX J0041.7+4134 & $14.38 \pm 0.49$ & 9 & $8.72 \pm 1.08$ & 1.8 & 0.29 & $1.65 \pm 0.21$ \\
\hline RX J0041.8+4021 & $24.25 \pm 0.76$ & 11 & $15.54 \pm 0.88$ & 5.4 & 0.12 & $1.56 \pm 0.10$ \\
\hline RX J0041.8+4113 & $0.43 \pm 0.09$ & 10 & $0.90 \pm 0.24$ & 32.9 & 0.73 & $0.48 \pm 0.16$ \\
\hline RX J0042.2+4019 & $40.38 \pm 1.23$ & 15 & $48.83 \pm 1.61$ & 2.9 & 0.06 & $0.83 \pm 0.04$ \\
\hline RX J0042.2+4039 & $2.18 \pm 0.19$ & 13 & $1.64 \pm 0.38$ & 18.4 & 0.41 & $1.33 \pm 0.33$ \\
\hline RX J0042.2+4055 & $2.71 \pm 0.18$ & 18 & $1.67 \pm 0.48$ & 1.9 & 0.32 & $1.62 \pm 0.48$ \\
\hline RX J0042.2+4101 & $9.53 \pm 0.32$ & 16 & $3.88 \pm 0.75$ & 0.7 & 0.12 & $2.46 \pm 0.48$ \\
\hline RX J0042.2+4112 & $9.04 \pm 0.25$ & 19 & $4.26 \pm 0.54$ & 8.0 & 1.37 & $2.12 \pm 0.28$ \\
\hline RX J0042.2+4117 & $2.18 \pm 0.16$ & 17 & $1.26 \pm 0.38$ & 5.0 & 0.86 & $1.73 \pm 0.54$ \\
\hline RX J0042.2+4118 & $9.71 \pm 0.32$ & 14 & $3.23 \pm 0.51$ & 9.5 & 1.63 & $3.01 \pm 0.49$ \\
\hline$\star \mathrm{RX}$ J0042.3+4113 & $5.02 \pm 0.23$ & 20 & $4.93 \pm 0.54$ & 9.1 & 1.55 & $1.02 \pm 0.12$ \\
\hline$\star \mathrm{RX} \mathrm{J} 0042.3+4115$ & $16.06 \pm 0.41$ & 23 & $6.88 \pm 0.61$ & 1.0 & 0.17 & $2.33 \pm 0.21$ \\
\hline RX J0042.4+4104 & $4.78 \pm 0.22$ & 28 & $3.07 \pm 0.71$ & 6.4 & 0.99 & $1.56 \pm 0.37$ \\
\hline RX J0042.4+4112 & $5.92 \pm 0.25$ & 27 & $3.38 \pm 0.52$ & 5.2 & 0.89 & $1.75 \pm 0.28$ \\
\hline RX J0042.4+4125 & $1.13 \pm 0.13$ & 30 & $1.71 \pm 0.47$ & 38.7 & 0.85 & $0.66 \pm 0.20$ \\
\hline RX J0042.5+4103 & $1.34 \pm 0.13$ & 37 & $2.61 \pm 0.85$ & 65.8 & 1.45 & $0.51 \pm 0.17$ \\
\hline$\star \mathrm{RX} \mathrm{J} 0042.5+4113$ & $4.15 \pm 0.22$ & 34 & $3.05 \pm 0.50$ & 7.1 & 1.22 & $1.36 \pm 0.23$ \\
\hline$\star \mathrm{RX} J 0042.5+4116$ & $6.01 \pm 0.21$ & 32 & $3.62 \pm 0.51$ & 6.8 & 1.16 & $1.66 \pm 0.24$ \\
\hline$\star \mathrm{RX} \mathrm{J} 0042.5+4119$ & $3.30 \pm 0.20$ & 33 & $0.83 \pm 0.28$ & 9.2 & 1.58 & $3.97 \pm 1.35$ \\
\hline RX J0042.5+4132 & $1.69 \pm 0.16$ & 38 & $1.56 \pm 0.29$ & 5.0 & 0.11 & $1.08 \pm 0.23$ \\
\hline RX J0042.6+4052 & $32.45 \pm 0.53$ & 51 & $9.16 \pm 1.01$ & 11.6 & 1.98 & $3.54 \pm 0.40$ \\
\hline$\star \mathrm{RX} \mathrm{J} 0042.6+4114$ & $3.75 \pm 0.17$ & 48 & $0.97 \pm 0.27$ & 9.1 & 1.56 & $3.87 \pm 1.09$ \\
\hline$\star \mathrm{RX}$ J0042.6+4115 & $42.30 \pm 0.38$ & 41 & $41.28 \pm 1.22$ & 6.9 & 1.19 & $1.02 \pm 0.03$ \\
\hline$\star \mathrm{RX}$ J0042.7+4111 & $4.13 \pm 0.21$ & 58 & $1.59 \pm 0.44$ & 3.7 & 0.63 & $2.60 \pm 0.73$ \\
\hline$\star$ RX J0042.7+4115 & $22.80 \pm 0.30$ & 52 & $1.62 \pm 0.31$ & 4.5 & 0.77 & $14.07 \pm 2.71$ \\
\hline$\star$ RX J0042.7+4116 & $29.89 \pm 0.28$ & 59 & $1.81 \pm 0.34$ & 8.6 & 1.47 & $16.51 \pm 3.14$ \\
\hline$\star$ RX J0042.8+4115 & $17.39 \pm 0.25$ & 63 & $8.04 \pm 0.63$ & 5.6 & 0.96 & $2.16 \pm 0.17$ \\
\hline$\star$ RX J0042.8+4118 & $9.68 \pm 0.33$ & 68 & $5.64 \pm 0.58$ & 6.4 & 1.09 & $1.72 \pm 0.19$ \\
\hline RX J0042.8+4125 & $3.95 \pm 0.22$ & 62 & $3.44 \pm 0.84$ & 6.2 & 1.06 & $1.15 \pm 0.29$ \\
\hline RX J0042.8+4131 & $18.62 \pm 0.45$ & 67 & $11.95 \pm 1.10$ & 4.6 & 0.78 & $1.56 \pm 0.15$ \\
\hline RX J0042.9+4111 & $3.84 \pm 0.21$ & 73 & $3.13 \pm 0.51$ & 5.4 & 0.92 & $1.23 \pm 0.21$ \\
\hline$\star \mathrm{RX} \mathrm{J} 0042.9+4117$ & $8.46 \pm 0.13$ & 72 & $0.77 \pm 0.27$ & 10.6 & 1.83 & $10.99 \pm 3.82$ \\
\hline$\star \mathrm{RX}$ J0042.9+4119 & $3.66 \pm 0.22$ & 76 & $1.64 \pm 0.46$ & 4.6 & 0.78 & $2.23 \pm 0.64$ \\
\hline RX J0042.9+4125 & $3.05 \pm 0.19$ & 70 & $3.77 \pm 0.34$ & 2.3 & 0.05 & $0.81 \pm 0.09$ \\
\hline$\star \mathrm{RX} \mathrm{J} 0043.0+4115$ & $8.97 \pm 0.31$ & 79 & $3.26 \pm 0.42$ & 1.7 & 0.30 & $2.75 \pm 0.36$ \\
\hline$\star \mathrm{RX} \mathrm{J} 0043.0+4117$ & $6.19 \pm 0.27$ & 80 & $1.92 \pm 0.35$ & 9.1 & 1.56 & $3.22 \pm 0.61$ \\
\hline RX J0043.1+4048 & $1.48 \pm 0.17$ & 81 & $1.04 \pm 0.42$ & 52.4 & 1.15 & $1.43 \pm 0.60$ \\
\hline RX J0043.1+4114 & $8.60 \pm 0.30$ & 83 & $7.51 \pm 0.61$ & 5.8 & 1.00 & $1.15 \pm 0.10$ \\
\hline RX J0043.1+4118 & $7.54 \pm 0.24$ & 82 & $2.03 \pm 0.31$ & 2.4 & 0.41 & $3.72 \pm 0.58$ \\
\hline RX J0043.2+4107 & $3.08 \pm 0.19$ & 85 & $4.27 \pm 0.94$ & 5.4 & 0.93 & $0.72 \pm 0.16$ \\
\hline RX J0043.3+4117 & $4.44 \pm 0.25$ & 88 & $1.31 \pm 0.34$ & 32.7 & 0.72 & $3.39 \pm 0.89$ \\
\hline RX J0043.3+4120 & $1.80 \pm 0.17$ & 87 & $2.15 \pm 0.52$ & 5.0 & 0.85 & $0.84 \pm 0.22$ \\
\hline RX J0043.3+4159 & $1.91 \pm 0.22$ & 86 & $0.96 \pm 0.29$ & 12.1 & 0.27 & $1.99 \pm 0.64$ \\
\hline RX J0043.4+4107 & $1.67 \pm 0.15$ & 90 & $1.64 \pm 0.28$ & 15.3 & 0.34 & $1.02 \pm 0.20$ \\
\hline RX J0043.4+4118 & $3.20 \pm 0.20$ & 89 & $2.48 \pm 0.60$ & 15.5 & 1.99 & $1.29 \pm 0.32$ \\
\hline RX J0043.5+4113 & $6.31 \pm 0.27$ & 91 & $6.24 \pm 1.02$ & 8.8 & 1.51 & $1.01 \pm 0.17$ \\
\hline RX J0043.6+4114 & $5.13 \pm 0.23$ & 92 & $4.73 \pm 0.90$ & 9.3 & 1.41 & $1.08 \pm 0.21$ \\
\hline RX J0043.7+4124 & $1.00 \pm 0.13$ & 93 & $2.86 \pm 0.74$ & 6.4 & 0.68 & $0.35 \pm 0.10$ \\
\hline RX J0043.8+4116 & $6.29 \pm 0.26$ & 94 & $4.05 \pm 0.78$ & 5.3 & 0.82 & $1.55 \pm 0.30$ \\
\hline RX J0043.9+4045 & $0.85 \pm 0.19$ & 95 & $1.64 \pm 0.50$ & 61.8 & 1.34 & $0.52 \pm 0.19$ \\
\hline RX J0044.4+4121 & $7.97 \pm 0.30$ & 97 & $8.59 \pm 1.09$ & 2.6 & 0.45 & $0.93 \pm 0.12$ \\
\hline RX J0044.9+4059 & $0.84 \pm 0.17$ & 98 & $2.07 \pm 0.73$ & 47.2 & 1.03 & $0.40 \pm 0.16$ \\
\hline RX J0045.2+4136 & $0.40 \pm 0.08$ & 99 & $1.03 \pm 0.27$ & 52.7 & 1.15 & $0.39 \pm 0.13$ \\
\hline RX J0045.4+4146 & $0.32 \pm 0.32$ & 100 & $0.71 \pm 0.23$ & 70.4 & 1.53 & $0.46 \pm 0.48$ \\
\hline RX J0045.6+4208 & $6.92 \pm 0.30$ & 101 & $4.99 \pm 0.86$ & 0.6 & 0.09 & $1.39 \pm 0.25$ \\
\hline RX J0045.7+4139 & $36.00 \pm 0.52$ & 102 & $43.18 \pm 3.15$ & 8.3 & 1.37 & $0.83 \pm 0.06$ \\
\hline RX J0046.1+4208 & $1.03 \pm 0.12$ & 103 & $0.95 \pm 0.24$ & 28.2 & 0.62 & $1.08 \pm 0.30$ \\
\hline RX J0046.4+4201 & $10.08 \pm 0.31$ & 105 & $5.52 \pm 0.89$ & 2.1 & 0.34 & $1.83 \pm 0.30$ \\
\hline RX J0046.4+4204 & $8.88 \pm 0.31$ & 104 & $8.56 \pm 0.48$ & 15.1 & 0.33 & $1.04 \pm 0.07$ \\
\hline RX J0046.9+4220 & $5.19 \pm 0.24$ & 107 & $3.54 \pm 0.53$ & 55.6 & 1.23 & $1.47 \pm 0.23$ \\
\hline RX J0048.0+4140 & $7.43 \pm 0.55$ & 108 & $4.46 \pm 0.89$ & 44.1 & 0.97 & $1.67 \pm 0.35$ \\
\hline
\end{tabular}


Table 8. Table of all optical and radio identifications.

\begin{tabular}{|c|c|c|c|c|}
\hline \multirow{2}{*}{$\begin{array}{l}\text { ROSAT } \\
\text { No. }\end{array}$} & \multirow[t]{2}{*}{ Obj.-Cl. } & \multirow[t]{2}{*}{ Identification } & \multicolumn{2}{|c|}{ Distance } \\
\hline & & & $\left({ }^{\prime \prime}\right)$ & $(\sigma)$ \\
\hline RX J0036.3+4053 & $\mathrm{GC}$ & $\mathrm{BA} 87(5)$ & 10.4 & 0.78 \\
\hline RX J0037.3+4043 & Star (F5) & SIMBAD( SAO 36516) & 13.0 & 1.49 \\
\hline RX J0038.0+4026 & Star & HA94(38232) & 6.1 & 1.21 \\
\hline RX J0038.4+4012 & Star & HA94(9276) & 6.9 & 1.38 \\
\hline RX J0038.4+4136 & EO & $87 \mathrm{~GB} 003540.8+412038$ & 34.9 & 1.51 \\
\hline RX J0038.6+4026 & Star (K0) & SIMBAD( SAO 36541) & 0.8 & 0.16 \\
\hline RX J0039.5+4008 & $\mathrm{EO}$ & B3 $0036+398$ & 10.2 & 1.83 \\
\hline RX J0039.6+4011 & Star & HA94(7297) & 4.1 & 0.47 \\
\hline RX J0039.7+4039 & Star & HA94(81094) & 11.0 & 1.25 \\
\hline RX J0040.1+4006 & Star & HA94(2646) & 15.9 & 1.17 \\
\hline RX J0040.1+4044 & Star & HA94(101871) & 12.7 & 1.47 \\
\hline RX J0040.1+4047 & Star & HA94(111215) & 7.0 & 1.40 \\
\hline RX J0040.2+4015 & Star & HA94(13652) & 2.4 & 0.48 \\
\hline RX J0040.2+4050 & $\mathrm{EO}$ & $87 \mathrm{~GB} 003730.5+403346$ & 6.0 & 1.11 \\
\hline RX J0040.3+4043 & $\mathrm{GC}$ & $\operatorname{MA} 94 \mathrm{a}(6)$ & 1.1 & 0.22 \\
\hline RX J0040.4+4050 & Star & HA94(119503) & 4.2 & 0.85 \\
\hline RX J0040.4+4129 & GC & $\mathrm{BA} 87(51)$ & 20.4 & 1.76 \\
\hline RX J0040.5+4033 & $\mathrm{GC}$ & MA94a(16) & 3.8 & 0.24 \\
\hline RX J0040.5+4034 & Star & HA94(61478) & 4.0 & 0.56 \\
\hline RX J0040.7+4055 & SNR & $\operatorname{DO} 80(7)$ & 11.3 & 0.64 \\
\hline RX J0040.8+4011 & Star $(\mathrm{K} 2 \mathrm{~V})$ & SIMBAD( GJ 28) & 5.4 & 1.04 \\
\hline RX J0040.9+4056 & Star & HA94(140092) & 4.7 & 0.94 \\
\hline RX J0041.3+4012 & EO & $87 \mathrm{~GB} 003844.0+395608$ & 4.8 & 0.26 \\
\hline RX J0041.3+4051 & Star & HA94(125532) & 3.6 & 0.71 \\
\hline RX J0041.3+4109 & Star & HA94(213303) & 56.0 & 1.86 \\
\hline RX J0041.4+4025 & Star & HA94(37411) & 14.9 & 1.63 \\
\hline RX J0041.5+4106 & SNR & MA95(3-041),DO80(11) & 3.2 & 0.45 \\
\hline RX J0041.6+4103 & Star & HA94(175678) & 14.9 & 1.90 \\
\hline RX J0041.6+4112 & Star & HA94(232216) & 8.1 & 1.61 \\
\hline RX J0041.7+4105 & Star & HA94(189885) & 0.4 & 0.08 \\
\hline RX J0041.7+4134 & GC & BA $87(98)$ & 1.6 & 0.28 \\
\hline RX J0041.8+4021 & EO & MRK 0957 & 11.0 & 1.93 \\
\hline RX J0041.9+4046 & SNR & MA95(2-021) & 26.0 & 1.67 \\
\hline RX J0042.0+4031 & Star & HA94(50780) & 8.0 & 0.82 \\
\hline RX J0042.0+4033 & Star & HA94(56227) & 17.3 & 1.78 \\
\hline RX J0042.0+4041 & Star (F5) & SIMBAD( HD 3914) & 5.5 & 1.05 \\
\hline RX J0042.0+4102 & $\mathrm{GC}$ & MA94a(130) & 9.0 & 1.76 \\
\hline RX J0042.1+4016 & Star & HA94(16045) & 10.1 & 1.10 \\
\hline RX J0042.2+4101 & GC & BA87(138),MA94a(159) & 2.0 & 0.40 \\
\hline RX J0042.2+4105 & Star & HA94(196074) & 4.1 & 0.81 \\
\hline RX J0042.2+4118 & Star & HA94(266451) & 6.4 & 1.29 \\
\hline RX J0042.3+4113 & GC & BA87(142),MA94a(164) & 7.3 & 1.43 \\
\hline RX J0042.3+4126 & Star & HA94(302803) & 10.5 & 1.21 \\
\hline RX J0042.3+4129 & $\mathrm{EO}$ & $87 \mathrm{~GB} 003934.6+411250$ & 11.9 & 1.55 \\
\hline RX J0042.4+4055 & Star & HA94(136397) & 8.7 & 1.74 \\
\hline RX J0042.4+4057 & GC & BA87(153),MA94a(173) & 6.2 & 1.22 \\
\hline RX J0042.4+4108 & Star & HA94(208922) & 10.7 & 1.37 \\
\hline RX J0042.4+4129 & Star & HA94(316946) & 2.4 & 0.32 \\
\hline RX J0042.5+4103 & GC & BA87(171),MA94a(196) & 2.7 & 0.53 \\
\hline RX J0042.5+4119 & GC & BA87(168),BA93(1),MA94a(192) & 4.6 & 0.91 \\
\hline RX J0042.5+4132 & GC & BA87(176),MA94a(205) & 2.5 & 0.39 \\
\hline RX J0042.6+4052 & $\mathrm{EO}$ & NGC $0221=$ M 32 & 9.7 & 1.90 \\
\hline RX J0042.6+4115 & GC & BA93(21) & 7.0 & 1.38 \\
\hline RX J0042.8+4125 & SNR & DO80(13) & 20.4 & 1.15 \\
\hline RX J0042.8+4131 & GC & BA87(196),MA94a(225) & 1.6 & 0.31 \\
\hline RX J0042.9+4119 & $\mathrm{GC}$ & $\mathrm{BA} 87(203)$ & 7.2 & 1.42 \\
\hline RX J0042.9+4125 & SNR & DO80(13) & 14.4 & 0.79 \\
\hline RX J0043.0+4110 & Star & HA94(223193) & 3.5 & 0.70 \\
\hline RX J0043.0+4115 & GC & BA $87(206)$ & 3.0 & 0.60 \\
\hline
\end{tabular}


Table 8. continued.

\begin{tabular}{|c|c|c|c|c|}
\hline \multirow{2}{*}{$\begin{array}{l}\text { ROSAT } \\
\text { No. }\end{array}$} & \multirow[t]{2}{*}{ Obj.-Cl. } & \multirow[t]{2}{*}{ Identification } & \multicolumn{2}{|c|}{ Distance } \\
\hline & & & $\left({ }^{\prime \prime}\right)$ & $(\sigma)$ \\
\hline RX J0043.0+4117 & $\mathrm{GC}$ & BA87(208) & 6.1 & 1.20 \\
\hline RX J0043.0+4121 & $\mathrm{GC}$ & BA87(207),MA94a(240) & 4.3 & 0.84 \\
\hline RX J0043.0+4130 & $\mathrm{GC}$ & MA94a $(236)$ & 4.1 & 0.80 \\
\hline RX J0043.1+4114 & $\mathrm{GC}$ & BA87(214),MA94a(251) & 3.0 & 0.60 \\
\hline RX J0043.2+4107 & $\mathrm{GC}$ & BA87(220),MA94a(257) & 5.5 & 1.08 \\
\hline RX J0043.2+4112 & $\mathrm{GC}$ & BA87(226),MA94a(269) & 11.5 & 0.94 \\
\hline RX J0043.2+4127 & $\mathrm{GC}$ & BA87(225),MA94a(266) & 6.0 & 1.02 \\
\hline RX J0043.3+4114 & Star & HA94(239273) & 16.0 & 1.53 \\
\hline RX J0043.4+4118 & SNR & MA95(2-032),DO80(15) & 14.2 & 1.63 \\
\hline RX J0043.5+4116 & Star & HA94(254312) & 1.1 & 0.09 \\
\hline RX J0043.6+4054 & $\mathrm{EO}$ & B3 $0040+406$ & 11.5 & 1.31 \\
\hline RX J0043.6+4114 & $\mathrm{GC}$ & BA87(246),MA94a(299) & 7.1 & 1.18 \\
\hline RX J0043.6+4126 & SNR & MA95(3-059),DO80(16) & 9.2 & 1.02 \\
\hline RX J0043.6+4138 & Star & HA94(375268) & 8.3 & 1.65 \\
\hline RX J0043.7+4128 & $\mathrm{GC}$ & MA94a(311) & 2.1 & 0.26 \\
\hline RX J0043.7+4136 & $\mathrm{GC}$ & MA94a(314) & 4.2 & 0.83 \\
\hline RX J0043.8+4106 & Star & HA94(196928) & 16.2 & 1.78 \\
\hline RX J0043.8+4111 & SNR & BW93(230A) & 10.7 & 0.85 \\
\hline RX J0043.8+4127 & $\mathrm{GC}$ & MA94a(317) & 39.9 & 0.83 \\
\hline RX J0043.9+4113 & SNR & MA95(2-038),DO80(18),BW93(252) & 24.7 & 1.78 \\
\hline RX J0043.9+4122 & $\mathrm{GC}$ & BA87(267),MA94a(334) & 3.0 & 0.58 \\
\hline RX J0043.9+4127 & Star & HA94(307124) & 7.6 & 1.53 \\
\hline RX J0043.9+4152 & SNR & MA95(2-037),DO80(17) & 11.7 & 1.06 \\
\hline RX J0043.9+4157 & $\mathrm{EO}$ & $87 \mathrm{~GB} 004113.4+414049$ & 7.4 & 0.87 \\
\hline RX J0044.0+4149 & SNR & MA95(3-072) & 19.5 & 1.51 \\
\hline RX J0044.2+4119 & SNR & MA95(3-079),BW93(327) & 9.1 & 1.28 \\
\hline RX J0044.2+4126 & Star & HA94(302441) & 7.5 & 0.75 \\
\hline RX J0044.4+4121 & GC & MA94a(387) & 1.9 & 0.36 \\
\hline RX J0044.4+4136 & $\mathrm{GC}$ & MA94a(380) & 5.8 & 1.14 \\
\hline RX J0044.6+4125 & SNR & MA95(3-086),BW93(490A) & 4.9 & 0.58 \\
\hline RX J0044.8+4129 & SNR & MA95(B-012),BW93(566) & 8.4 & 1.19 \\
\hline RX J0044.8+4229 & Star (G5) & SIMBAD( HD4194) & 6.6 & 0.56 \\
\hline RX J0045.1+4202 & Star & HA94(441416) & 6.4 & 1.28 \\
\hline RX J0045.2+4136 & SNR & MA95(2-048),BW93(717),DO80(19) & 11.9 & 1.23 \\
\hline RX J0045.2+4217 & Star & HA94(466304) & 14.8 & 1.48 \\
\hline RX J0045.4+4132 & GC & MA94a(447) & 15.5 & 1.85 \\
\hline RX J0045.4+4146 & SNR & MA95(1-013) & 17.3 & 1.54 \\
\hline RX J0045.5+4210 & Star & HA94(456238) & 7.8 & 1.48 \\
\hline RX J0045.7+4139 & $\mathrm{GC}$ & BA87(318),MA94a(468) & 5.8 & 1.08 \\
\hline RX J0045.9+4156 & Star & HA94(431022) & 6.1 & 1.21 \\
\hline RX J0045.9+4203 & Star & HA94(442137) & 8.5 & 1.10 \\
\hline RX J0045.9+4226 & Star & HA94(479836) & 8.1 & 1.61 \\
\hline RX J0046.0+4136 & Star & HA94(364424) & 20.4 & 1.78 \\
\hline RX J0046.2+4154 & Star & HA94(425498) & 6.3 & 1.26 \\
\hline RX J0046.4+4201 & GC & BA87(329),MA94a(487) & 3.0 & 0.53 \\
\hline RX J0046.6+4225 & Star & HA94(478682) & 3.8 & 0.76 \\
\hline RX J0046.7+4208 & $\mathrm{EO}$ & $87 \mathrm{~GB} 004359.8+415217$ & 6.1 & 1.20 \\
\hline RX J0046.7+4230 & Star & HA94(483376) & 18.9 & 1.86 \\
\hline RX J0047.0+4157 & Star $(F)$ & SIMBAD( HD4444) & 1.9 & 0.22 \\
\hline RX J0047.0+4201 & Star & HA94(440331) & 9.8 & 0.66 \\
\hline RX J0047.2+4202 & Star & HA94(441062) & 14.5 & 1.92 \\
\hline RX J0047.4+4220 & Star & HA94(472654) & 7.7 & 1.54 \\
\hline RX J0047.4+4221 & Star & HA94(474007) & 5.6 & 1.12 \\
\hline RX J0047.7+4201 & Star & HA94(439698) & 12.8 & 1.46 \\
\hline RX J0048.4+4157 & Star (F8) & SIMBAD( SAO 36677) & 7.4 & 1.14 \\
\hline
\end{tabular}

